\title{
Enantioselective Intramolecular Michael Addition of Nitronates onto Conjugated Esters: Access to Cyclic $\gamma$-Amino Acids with up to Three Stereocentres
}

\author{
William J. Nodes, ${ }^{1}$ David R. Nutt ${ }^{2}$ and Alexander J. A. Cobb* ${ }^{* 1}$ \\ ${ }^{1}$ School of Pharmacy and ${ }^{2}$ Department of Chemistry, University of Reading, Whiteknights, Reading, Berks RG6 \\ GAD UK
}

\section{Supporting Information}

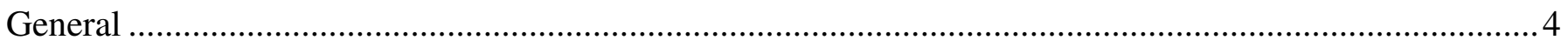

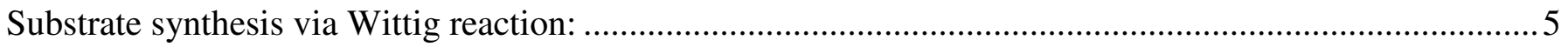

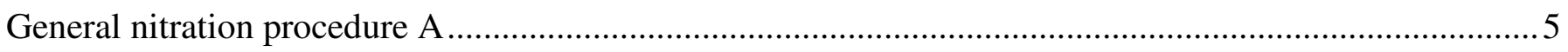

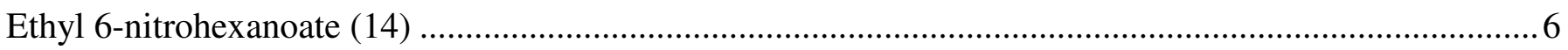

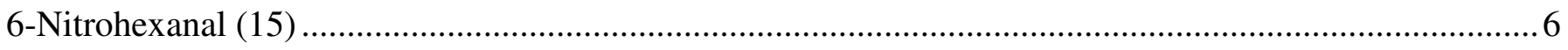

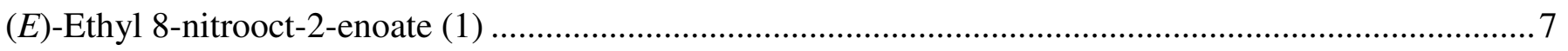

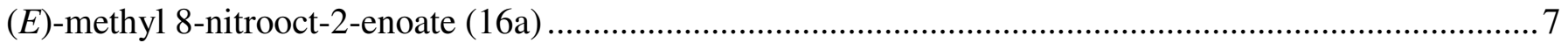

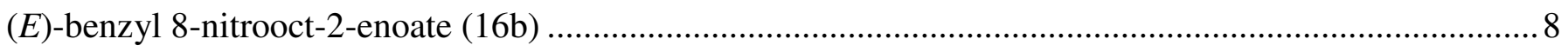

(E)-ethyl 2-methyl-8-nitrooct-2-enoate (16c) ..................................................................................

Substrate synthesis via Horner-Wadsworth or Still-Gennari reaction .................................................... 9

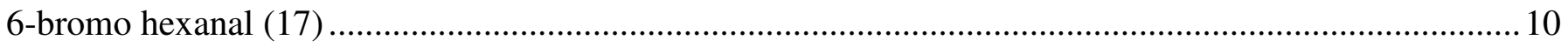

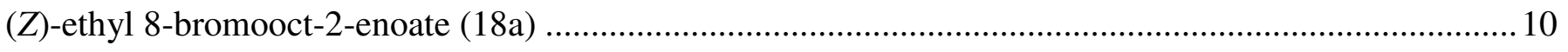

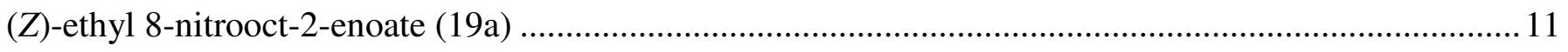

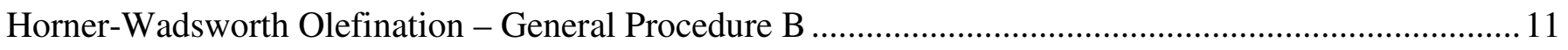

(E)-Ethyl 2-benzyl-8-bromooct-2-enoate and (Z)-Ethyl 2-benzyl-8-bromooct-2-enoate (18b) ................... 12

(E)-Ethyl 2-benzyl-8-nitrooct-2-enoate and (Z)-Ethyl 2-benzyl-8-nitrooct-2-enoate (19b) ........................ 12

(Z)-Methyl 2-(N-tert-butoxycarbonyl)-amino-8-bromooct-2-enoate (18c) ………………....................... 13

(Z)-Methyl 2-(N-tert-butoxycarbonyl)-amino-8-nitrooct-2-enoate (19c) ……………................................ 14

(Z)-Methyl 2-(N-benzylcarbonyl)-amino-8-bromooct-2-enoate (18d) …….............................................. 14 
(Z)-Methyl 2-(N-benzylcarbonyl)-amino-8-nitrooct-2-enoate (19d)

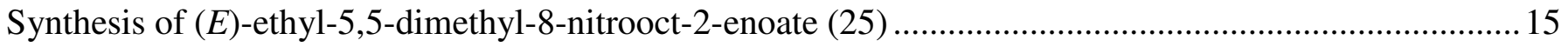

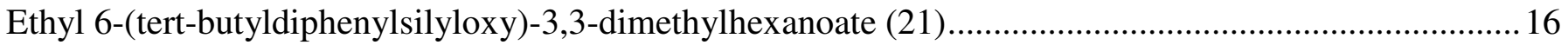

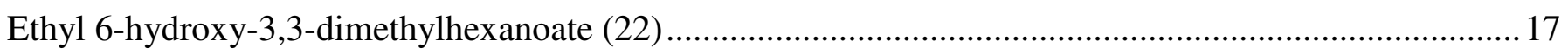

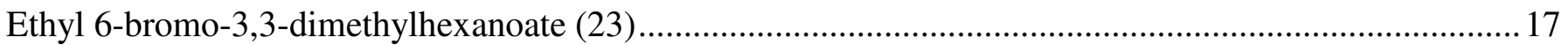

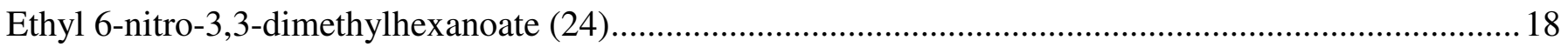

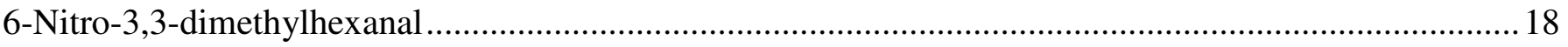

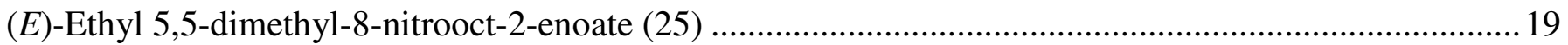

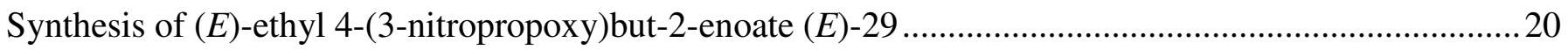

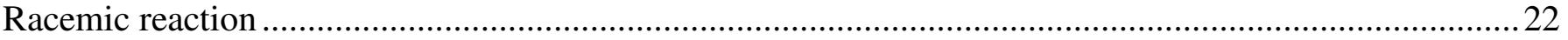

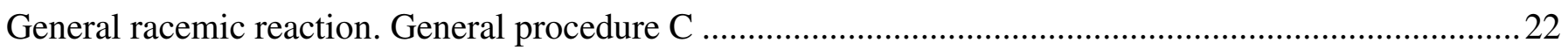

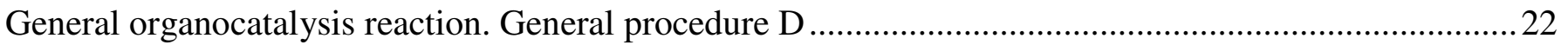

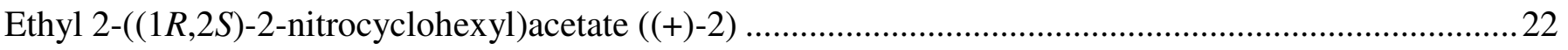

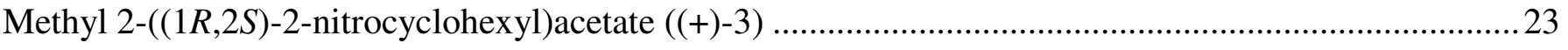

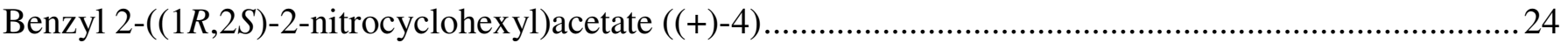

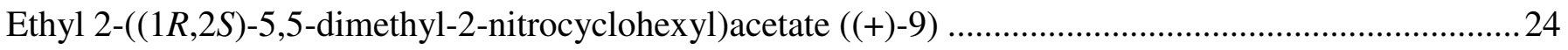

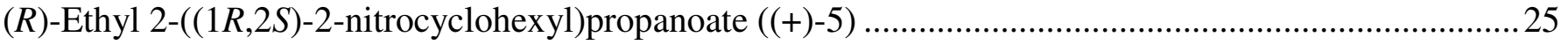

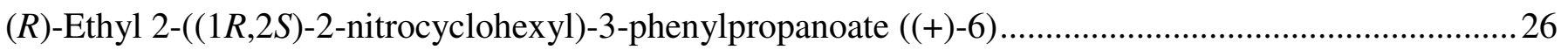

$(R)$-Ethyl 2-(tert-butoxyaminocarbonyl)-2-((1R,2S)-2-nitrocyclohexyl)acetate ((-)-10) .........................22

(R)-Methyl 2-(benzyloxyaminocarbonyl)-2-((1R,2S)-2-nitrocyclohexyl)acetate ((-)-7) .........................27

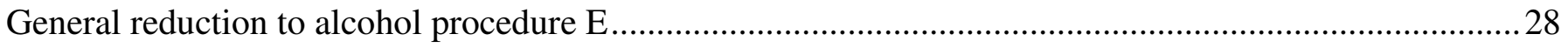

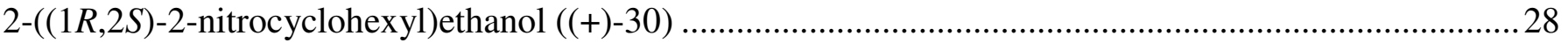

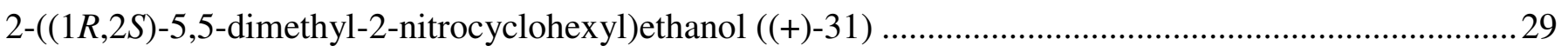

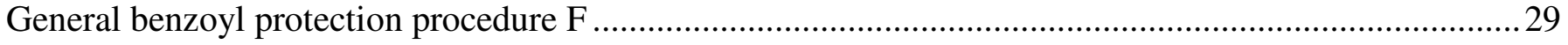

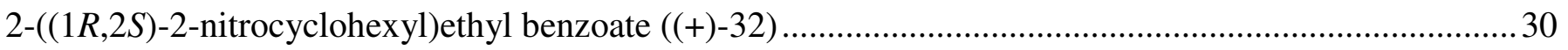


Absolute configuration determined by Nef reaction and comparison of products optical rotation with

literature value. 31

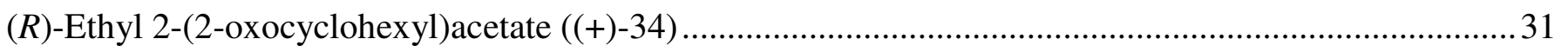

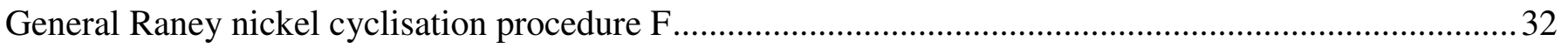

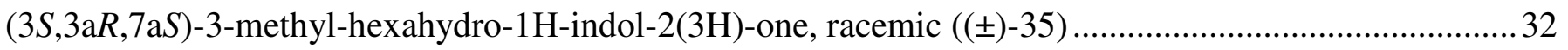

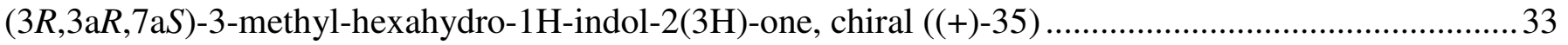

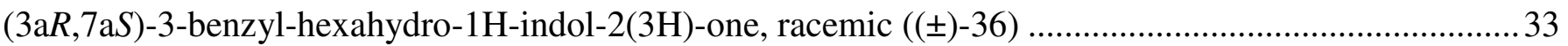

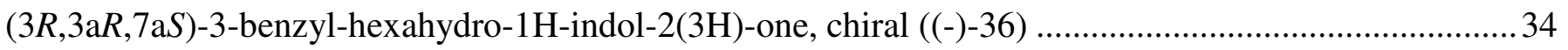

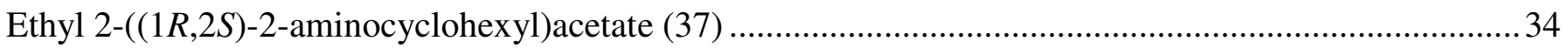

Ethyl 2-((1R,2S)-2-((S)-2-(benzyloxycarbonyl)-3-methylbutanamido)cyclohexyl)acetate ((-)-38) ...........35

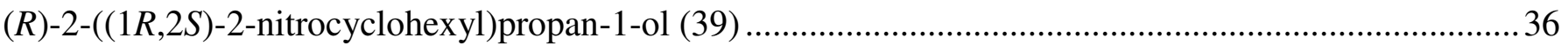

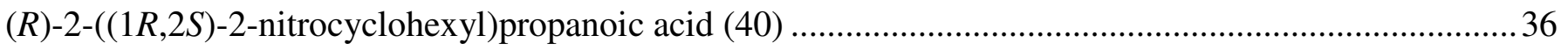

(S)-Methyl 3-methyl-2-((R)-2-((1R,2S)-2-nitrocyclohexyl)propanamido)butanoate $((+)-41) \ldots \ldots \ldots \ldots \ldots \ldots . . . . . . .37$

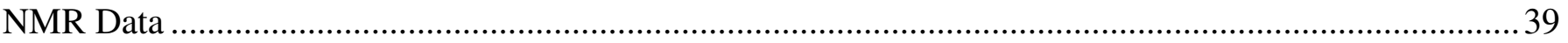

X-Ray Crystal Structure and Associated Data for Compound X .................................................... 87 


\section{General}

${ }^{1} \mathrm{H}$ NMR spectra were recorded in either deuterated chloroform $\left(\mathrm{CDCl}_{3}\right)$ or methanol (MeOD), using a Bruker DPX $250(250 \mathrm{MHz})$ spectrometer, a Bruker DPX $400(400 \mathrm{MHz})$ spectrometer or a Bruker Nanobay $400(400 \mathrm{MHz})$ spectrometer. Chemical shifts $(\delta)$ are quoted in parts per million using the abbreviations: s, singlet; $d$, doublet;; dd, double doublet; t, triplet; q, quartet; m, multiplet. All coupling constants were rounded to the nearest $0.5 \mathrm{~Hz}$.

${ }^{13} \mathrm{C} \mathrm{NMR}$ spectra were recorded at $63 \mathrm{MHz}$ or $100 \mathrm{MHz}$ in deuterated chloroform $\left(\mathrm{CDCl}_{3}\right)$ or methanol (MeOD) on a Bruker DPX 250, DPX 400 or Nanobay 400 respectively. Chemical shifts (ठ) are quoted in parts per million.

Infrared spectra were recorded on a Perkin-Elmer FT-IR spectrometer as a thin film. The absorptions are quoted in wavenumbers $\left(\mathrm{cm}^{-1}\right)$.

Mass spectrometry data was recorded on either a VG Autospec mass spectrometer using chemical ionisation $(\mathrm{Cl})$ conditions using gaseous ammonia as the ionising source, or a Thermo Scientific LTQ Orbitrap XL using electrospray ionisation (ESI) conditions.

Specific optical rotations $\left([\alpha]_{D}\right)$ were recorded at the sodium $D$ line in chloroform and are quoted in units of $10^{-1}$ deg $\mathrm{cm}^{2} \mathrm{~g}^{-1}$. Solution concentrations (c) are given in the units of $10^{-2} \mathrm{gmL}^{-1}$. Readings were taken using a Perkin-Elmer 341 polarimeter.

Melting points were determined on a Stuart SMP3 melting point apparatus and are uncorrected. Thin layer chromatography (tlc) was performed on Merck aluminium backed plates coated with $0.2 \mathrm{~mm}$ silica gel $60 \mathrm{~F}_{254}$. The spots were visualised using UV light $(254 \mathrm{~nm})$ and then permanent staining by solutions of Permangenate. Column chromatography was carried out using silica gel $60(40-63 \mu \mathrm{m})$. HPLC analysis was determined on an Agilent Technologies 1200 Series HPLC, using a ratio of hexanes and propan-2-ol as the elutent, using either a Chiralpak AD-H column 
$(0.46 \mathrm{~cm} ø \mathrm{X} 25 \mathrm{~cm})$ or a Chrialcel OD column $(0.46 \mathrm{~cm} \varnothing \mathrm{X} 25 \mathrm{~cm})$, detection by UV at $210 \mathrm{~nm}$ or $254 \mathrm{~nm}$.

X-ray data was collected on a Oxford Gemini S-ultra defractometer using Ka $(\lambda=1.54180 \AA \hat{)})$ radiation.

Anhydrous solvents were supplied as Sureseal ${ }^{\circledR}$ bottles by Aldrich. All chemicals were supplied by Aldrich, Fisher and Acros and were used as supplied unless otherwise stated.

Compound numbering within this supporting information continue from the manuscript.

\section{Substrate synthesis via Wittig reaction:}
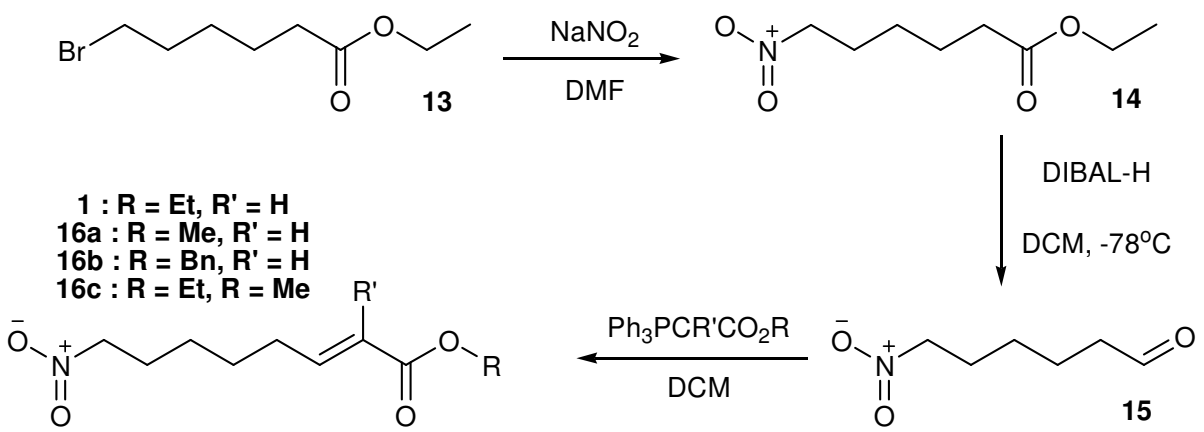

\section{General nitration procedure A}

To a solution of bromo-compound (1 eq.) in dimethyl formamide $(0.1 \mathrm{M})$ was added sodium nitrite (1.5 eq.), this solution was stirred until TLC analysis showed the consumption of the majority of the bromo-compound. The reaction was then poured into ice cold water, extracted with diethyl ether, the aqueous phase was washed with diethyl ether, the combined organic layers were dried over $\mathrm{MgSO}_{4}$, filtered and concentrated under reduced pressure to afford the crude nitro compound. 


\section{Ethyl 6-nitrohexanoate (14)}

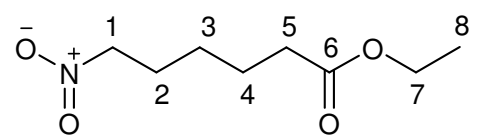

Following general procedure A, ethyl 6-bromo hexanoate $(15.0 \mathrm{~g}$, $67.08 \mathrm{mmol}$ ) gave crude product as a light yellow oil. Purification by column chromatography (silica gel, diethyl ether/hexane, 3/17) afforded the title compound as a clear colourless oil $(6.60 \mathrm{~g}, 52 \%, 34.88 \mathrm{mmol}) .{ }^{1} \mathrm{H}$ NMR $\left(250 \mathrm{MHz}, \mathrm{CDCl}_{3}\right) 1.23-1.29(3 \mathrm{H}, \mathrm{t}, \mathrm{J}=$ 7.5 Hz, H-8), 1.37-1.49 (2 H, m, H-3), 1.63-1.75 (2 H, m, H-4), 1.98-2.10 (2 H, m, H-2), 2.30-2.36 (2 H, t, J = 7.5 Hz, H-5), 4.09-4.18 (2 H, q, J = 7.5 Hz, H-7), 4.37-4.42 (2 H, t, J = 7.5 Hz, H-1); ${ }^{13} \mathrm{C}$ NMR $\left(62.8 \mathrm{MHz}, \mathrm{CDCl}_{3}\right) 14.2$ 24.1, 25.7, 27.1, 33.8, 60.4, 75.4, 173.6; IR $\left(\mathrm{cm}^{-1}\right) 2936(\mathrm{w})$, 1957 (w), 1730 (s), 1552 (s), 1436 (w), 1376 (m), $1180(\mathrm{~m}), 1096$ (m), 1032 (m); HRMS required for $\left(\mathrm{C}_{8} \mathrm{H}_{16} \mathrm{NO}_{4}{ }^{+}\right)$190.1079; found 190.1078 .

\section{6-Nitrohexanal (15)}

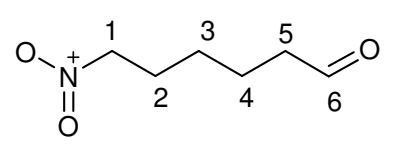

To a solution of ethyl-6-nitrohexanoate $14(6.60 \mathrm{~g}, 34.88 \mathrm{mmol})$ in anhydrous DCM $(250 \mathrm{~mL})$ at $-78{ }^{\circ} \mathrm{C}$ was added DIBAL-H $(38 \mathrm{~mL}, 38.37$

mmol, $1 \mathrm{M}$ solution in hexanes). After 1 hour at $-78{ }^{\circ} \mathrm{C}$ TLC analysis showed consumption of starting material. The reaction was quenched with aqueous hydrochloric acid $(1 \mathrm{M}, 50 \mathrm{~mL})$ and water $(100 \mathrm{~mL})$, and the aqueous layer was extracted with dichloromethane $(2 \times 50 \mathrm{~mL})$. The combined organic layers were washed with water, dried over $\mathrm{MgSO}_{4}$, filtered and concentrated under reduced pressure to afford crude the title compound as a light yellow oil. ${ }^{1} \mathrm{H}$ NMR (250 $\left.\mathrm{MHz}, \mathrm{CDCl}_{3}\right)$ 1.37-1.49 (2 H, m, H-3), 1.60-1.75 (2 H, m, H-4), 1.98-2.10 (2 H, m, H-2), 2.46-2.52 $(2 \mathrm{H}, \mathrm{dt}, J=2.5 \& 7.5 \mathrm{~Hz}, \mathrm{H}-5), 4.37-4.43(2 \mathrm{H}, \mathrm{t}, J=7.5 \mathrm{~Hz}, \mathrm{H}-1), 9.78(1 \mathrm{H}, \mathrm{t}, J=2.5 \mathrm{~Hz}, \mathrm{H}-6)$; ${ }^{13} \mathrm{C}$ NMR $\left(62.8 \mathrm{MHz}, \mathrm{CDCl}_{3}\right)$ 21.2, 25.8, 27.2, 43.4, 75.4, 201.7. 


\section{(E)-Ethyl 8-nitrooct-2-enoate (1)}

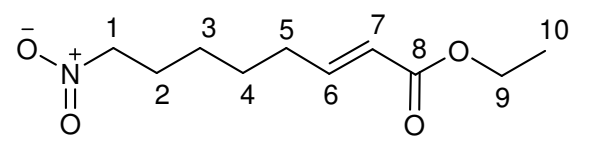

To a solution of crude 6-nitrohexanal $15(1.41 \mathrm{~g}, 9.71 \mathrm{mmol})$ in dichloromethane (100 $\mathrm{mL})$ was added in one portion (carbethoxymethylene)triphenylphosphorane $(3.87 \mathrm{~g}, 10.68 \mathrm{mmol})$. The reaction mixture was left to stir for 18 hours, quenched with saturated ammonium chloride $(50 \mathrm{~mL})$, the aqueous layer was extracted with dichloromethane $(2 \times 50 \mathrm{~mL})$. The combined organic layers were washed with water, dried over $\mathrm{MgSO}_{4}$, filtered and concentrated under reduced pressure to afford the crude product as a viscous yellow oil. Purification by column chromatography (silica gel, diethyl ether/hexane, 1/3) afforded the title compound as a light yellow oil $(1.43 \mathrm{~g}, 68 \%, 6.64 \mathrm{mmol}) .{ }^{1} \mathrm{H}$ NMR (250 MHz, $\left.\mathrm{CDCl}_{3}\right) 1.32(3 \mathrm{H}, \mathrm{t}, J=7.5 \mathrm{~Hz}, \mathrm{H}-10), 1.38-1.60(4 \mathrm{H}, \mathrm{m}, \mathrm{H}-3 \& 4), 1.97-2.09$ (2 $\mathrm{H}, \mathrm{m}, \mathrm{H}-2), 2.17-2.33(2 \mathrm{H}, \mathrm{m}, \mathrm{H}-5), 4.11-4.20(2 \mathrm{H}, \mathrm{q}, \mathrm{J}=7.5 \mathrm{~Hz}, \mathrm{H}-9), 4.39(2 \mathrm{H}, \mathrm{t}, \mathrm{J}=7.5 \mathrm{~Hz}$, $\mathrm{H}-1), 5.78-5.86(1 \mathrm{H}, \mathrm{dt}, J=2.5 \& 15 \mathrm{~Hz}, \mathrm{H}-7), 6.87-6.99(1 \mathrm{H}, \mathrm{dt}, J=7.5 \& 15 \mathrm{~Hz}, \mathrm{H}-6) ;{ }^{13} \mathrm{C}$ NMR $\left(62.8 \mathrm{MHz}, \mathrm{CDCl}_{3}\right)$ 14.3, 25.8, 27.1, 27.3, 31.7, 60.3, 75.5, 121.9, 148.2; IR $\left(\mathrm{cm}^{-1}\right) 3418$ (m), 2934 (s), 1958 (w), 1717 (s), 1654 (s), 1550 (s), 1463 (m), 1435 (m), 1369 (s), 1308 (s), 1268 (s), 1155 (s), 1117 (m), 1096 (m), 1044 (s), 983 (s), 865 (w), 736 (w), 711 (w); HRMS required for $\left(\mathrm{C}_{10} \mathrm{H}_{18} \mathrm{NO}_{4}{ }^{+}\right) 216.1236$; found 216.1235.

\section{(E)-methyl 8-nitrooct-2-enoate (16a)}

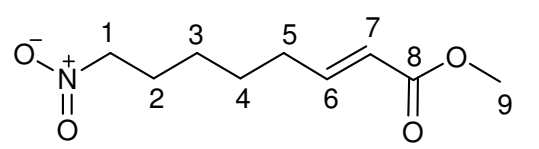

To a solution of crude 6-nitrohexanal $15(0.64 \mathrm{~g}, 4.44 \mathrm{mmol})$ in dichloromethane $(20 \mathrm{~mL})$ was added in one portion (carbmethoxymethylene)triphenylphosphorane $(1.63 \mathrm{~g}, 4.88 \mathrm{mmol})$. The reaction mixture was left to stir for 18 hours, quenched with saturated ammonium chloride $(10 \mathrm{~mL})$ and the aqueous layer was extracted with dichloromethane $(2 \times 15 \mathrm{~mL})$. The combined organic layers were washed with water, dried over $\mathrm{MgSO}_{4}$, filtered and concentrated under reduced pressure to afford the crude product as a viscous yellow oil. Purification by column chromatography (silica 
gel, diethyl ether/hexane, 1/3) afforded the title compound as a clear colourless oil $(0.61 \mathrm{~g}, 68$ \%, $3.02 \mathrm{mmol}) .{ }^{1} \mathrm{H}$ NMR $\left(250 \mathrm{MHz}, \mathrm{CDCl}_{3}\right)$ 1.38-1.52 (4 H, m, H-3 \& 4), 1.97-2.09 (2 H, quin, $J=$ $7.5 \mathrm{~Hz}, \mathrm{H}-2), 2.19-2.28$ (2 h, dq, J = 1 \& $7.5 \mathrm{~Hz}, \mathrm{H}-5), 3.73(3 \mathrm{H}, \mathrm{s}, \mathrm{H}-9), 4.36-4.42(2 \mathrm{H}, \mathrm{t}, J=$ $7.5 \mathrm{~Hz}), 5.79-5.87(1 \mathrm{H}, \mathrm{dt}, J=2.5 \& 15 \mathrm{~Hz}, \mathrm{H}-7), 6.88-7.00(1 \mathrm{H}, \mathrm{dt}, J=7.5 \& 12.5 \mathrm{~Hz}, \mathrm{H}-6) ;{ }^{13} \mathrm{C}$ $\operatorname{NMR}\left(62.8 \mathrm{MHz}, \mathrm{CDCl}_{3}\right)$ 25.8, 27.1, 27.3, 31.7, 51.5, 75.5, 121.4, 148.5; IR (cm-1) 2948 (s), $2861(\mathrm{~m}), 1716(\mathrm{~s}), 1657(\mathrm{~s}), 1556(\mathrm{~s}), 1434(\mathrm{~s}), 1384(\mathrm{~m}), 1311(\mathrm{~m}), 1273(\mathrm{~m}), 1198(\mathrm{~m}), 1120$ (m), $1041(\mathrm{~m}), 984(\mathrm{~m}), 862(\mathrm{w}), 719(\mathrm{w}), 614(\mathrm{w})$; HRMS required for $\left(\mathrm{C}_{9} \mathrm{H}_{16} \mathrm{NO}_{4}{ }^{+}\right)$202.1079; found 202.1078 .

\section{(E)-benzyl 8-nitrooct-2-enoate (16b)}

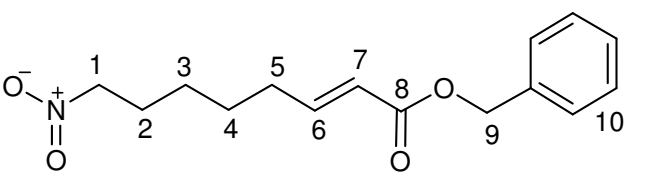

To a solution of crude 6-nitrohexanal $15(0.77 \mathrm{~g}, 5.28$ $\mathrm{mmol})$ in dichloromethane $(20 \mathrm{~mL})$ was added in one portion (carbbenzoxymethylene)triphenylphosphorane $(2.38 \mathrm{~g}, 5.81 \mathrm{mmol})$. The reaction mixture was left to stir for 18 hours, quenched with saturated ammonium chloride $(10 \mathrm{~mL})$ and the aqueous layer was extracted with dichloromethane $(2 \times 15 \mathrm{~mL})$. The combined organic layers were washed with water, dried over $\mathrm{MgSO}_{4}$, filtered and concentrated under reduced pressure to afford the product as a crude viscous yellow oil. Purification by column chromatography (silica gel, diethyl ether/hexane, 1/3) afforded the title product as a clear colourless oil $(0.77 \mathrm{~g}, 53 \%$, $2.79 \mathrm{mmol}) .{ }^{1} \mathrm{H}$ NMR (250 MHz, $\left.\mathrm{CDCl}_{3}\right)$ 1.38-1.52 (4 H, m, H-3 \& 4), 1.96-2.08 (2 H, quin, $J=$ $7.5 \mathrm{~Hz}, \mathrm{H}-2), 2.19-2.28(2 \mathrm{~h}, \mathrm{dq}, J=1 \& 7.5 \mathrm{~Hz}, \mathrm{H}-5), 4.35-4.41(2 \mathrm{H}, \mathrm{t}, J=7.5 \mathrm{~Hz}), 5.18(2 \mathrm{H}, \mathrm{s}$, $\mathrm{H}-9)$ 5.84-5.91 (1 H, dt, J = $2.5 \& 15 \mathrm{~Hz}, \mathrm{H}-7), 6.92-7.04(1 \mathrm{H}, \mathrm{dt}, J=7.5$ \& $12.5 \mathrm{~Hz}, \mathrm{H}-6), 7.31-$ $7.40(5 \mathrm{H}, \mathrm{m}, \mathrm{H}-10) ;{ }^{13} \mathrm{C} \operatorname{NMR}\left(62.8 \mathrm{MHz}, \mathrm{CDCl}_{3}\right) 25.8,27.1,27.3,31.6,66.2,75.5,121.6$, 128.3, 128.6, 149.0; IR (cm $\left.{ }^{-1}\right) 2932$ (s), 2860 (m), 1715 (s), 1653 (s), 1556 (s), $1496(\mathrm{~m}), 1455$ $(\mathrm{m}), 1433(\mathrm{~m}), 1380(\mathrm{~s}), 1265$ (s), $1182(\mathrm{~s}), 1118(\mathrm{~m}), 1015(\mathrm{~m}), 909(\mathrm{w}), 740(\mathrm{~m}), 698(\mathrm{~s}), 603$ (w); HRMS required for $\left(\mathrm{C}_{15} \mathrm{H}_{19} \mathrm{NaNO}_{4}\right) 300.1212$; found 300.1217 . 


\section{(E)-ethyl 2-methyl-8-nitrooct-2-enoate (16c)}<smiles>CCOC(=O)C(C)=CCCCCCC(C)[N+](=O)[O-]</smiles>

To a solution of crude 6-nitrohexanal $15(0.54 \mathrm{~g}, 3.70 \mathrm{mmol})$ in

dichloromethane $(20 \mathrm{~mL})$ was added in one portion ethyl 2(triphenylphosphoranylidene)propanoate $(1.48 \mathrm{~g}, 4.07 \mathrm{mmol})$. The reaction mixture was left to stir for 18 hours, quenched with saturated ammonium chloride $(100 \mathrm{~mL})$ and the aqueous layer was extracted with dichloromethane $(2 \times 50 \mathrm{~mL})$. The combined organic layers were washed with water, dried over $\mathrm{MgSO}_{4}$, filtered and concentrated under reduced pressure to afford the crude product as a viscous yellow oil. Purification by column chromatography (silica gel, diethyl ether/hexane, 1/3) afforded the title compound as a clear colourless oil $(0.48 \mathrm{~g}, 57 \%, 2.09$ mmol). ${ }^{1} \mathrm{H}$ NMR $\left(250 \mathrm{MHz}, \mathrm{CDCl}_{3}\right) 1.27-1.33(3 \mathrm{H}, \mathrm{t}, \mathrm{J}=7.5 \mathrm{~Hz}, \mathrm{H}-11), 1.36-1.58(4 \mathrm{H}, \mathrm{m}, \mathrm{H}-$ 3\&4), $1.83(3 \mathrm{H}, \mathrm{t}, J=1 \mathrm{~Hz}, \mathrm{H}-8), 1.98-2.09(2 \mathrm{H}$, quni, $J=7.5 \mathrm{~Hz}, \mathrm{H}-2), 2.16-2.24(2 \mathrm{H}, \mathrm{q}, J=$ 7.5 Hz, H-5), 4.15-4.23 (2 H, q, J = 7.5 Hz, H-10), 4.36-4.42 (2 H, t, J= 7.5 Hz, H-1), 6.69-6.75 $(1 \mathrm{H}, \mathrm{dt}, J=1 \& 7.5 \mathrm{~Hz}, \mathrm{H}-6) ;{ }^{13} \mathrm{C}$ NMR $\left(62.8 \mathrm{MHz}, \mathrm{CDCl}_{3}\right)$ 12.4, 14.3, 21.9, 26.0, 27.2, 27.9, 28.3, 60.5, 75.5, 141.1; IR (cm $\left.{ }^{-1}\right) 2933(\mathrm{~m}), 1706$ (s), $1648(w), 1552(\mathrm{~s}), 1436(\mathrm{w}), 1383(\mathrm{w})$, $1261(\mathrm{~m}), 1175(\mathrm{w}), 1133(\mathrm{~m}), 1096(\mathrm{~m}), 1028(\mathrm{w}), 747(\mathrm{w})$; HRMS required for $\left(\mathrm{C}_{11} \mathrm{H}_{19} \mathrm{NaNO}_{4}\right)$ 252.1212; found 252.1211.

\section{Substrate synthesis via Horner-Wadsworth or Still-Gennari reaction}

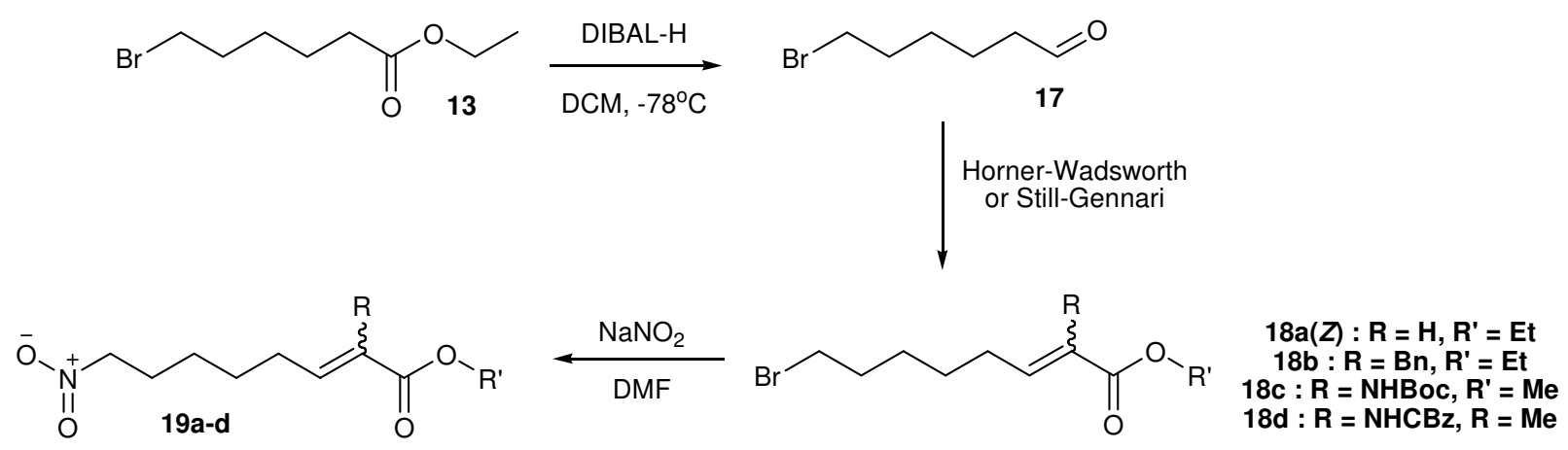




\section{6-bromo hexanal (17)}

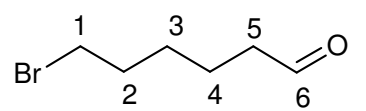

To a solution of ethyl-6-bromohexanoate $13(1.34 \mathrm{~g}, 6.02 \mathrm{mmol})$ in dichloromethane $(250 \mathrm{~mL})$ at $-78{ }^{\circ} \mathrm{C}$ was added DIBAL-H $(6.6 \mathrm{~mL}, 6.62$ mmol, $1 \mathrm{M}$ solution in hexanes). After 1 hour at $-78{ }^{\circ} \mathrm{C}$ TLC analysis showed consumption of starting material. The reaction was quenched with aqueous hydrochloric acid $(1 \mathrm{M}, 50 \mathrm{~mL})$ and water $(100 \mathrm{~mL})$ and the aqueous layer was extracted with dichloromethane $(2 \times 50 \mathrm{~mL})$. The combined organic layers were washed with water, dried over $\mathrm{MgSO}_{4}$, filtered and concentrated under reduced pressure to afford the crude title compound as a light yellow oil, which was used without further purification. ${ }^{1} \mathrm{H}$ NMR $\left(250 \mathrm{MHz}, \mathrm{CDCl}_{3}\right)$ 1.37-1.49 $(2 \mathrm{H}, \mathrm{m}, \mathrm{H}-3), 1.60-1.75(2 \mathrm{H}$, m, H-4) 1.98-2.10 (2 H, quin, $J=7.5 \mathrm{~Hz}, \mathrm{H}-2), 2.46-2.52(2 \mathrm{H}, \mathrm{dt}, J=2.5$ \& $7.5 \mathrm{~Hz}, \mathrm{H}-5), 4.37-$ $4.43(2 \mathrm{H}, \mathrm{t}, J=7.5 \mathrm{~Hz}, \mathrm{H}-1), 9.78(1 \mathrm{H}, \mathrm{t}, J=1.0 \mathrm{~Hz}, \mathrm{H}-6)$.

\section{(Z)-ethyl 8-bromooct-2-enoate (18a)}

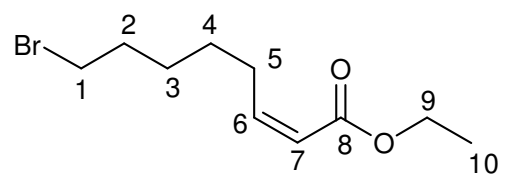

To a solution of ethyl [bis(2,2,2-trifluoroethoxy)phosphoryl]acetate

$(3.0 \mathrm{~g}, 9.03 \mathrm{mmol})$ in tetrahydrofuran $(60 \mathrm{~mL})$ at $-78^{\circ} \mathrm{C}$ was added, 18-crown-6-ether $(7.96 \mathrm{~g}, 30.01 \mathrm{mmol})$ and KHMDS $(18 \mathrm{~mL}, 9.03 \mathrm{mmol})$. This solution was stirred for 10 minutes at $-78^{\circ} \mathrm{C}$ before the addition of the crude 6-bromo hexanal $17(1.08 \mathrm{~g}, 6.02$ mmol). The resulting mixture was left to stir overnight. After this time the reaction was quenched with aqueous ammonium chloride $(20 \mathrm{~mL})$, the aqueous layer was washed with diethyl ether $(2 \mathrm{x}$ $25 \mathrm{~mL}$ ) and the combined organic fractions dried over $\mathrm{MgSO}_{4}$, filtered and concentrated under reduced pressure to afford the crude product as a yellow oil, Purification by column chromatography (silica gel, diethyl ether/hexane, 1/3) afforded the title compound as a clear colourless oil $(0.75 \mathrm{~g}, 50 \%, 3.01 \mathrm{mmol}) .{ }^{1} \mathrm{H}$ NMR $\left(250 \mathrm{MHz}, \mathrm{CDCl}_{3}\right) 1.26-1.32(3 \mathrm{H}, \mathrm{t}, \mathrm{J}=7.5$ $\mathrm{Hz}, \mathrm{H}-10), 1.42-1.54$ (4 H, m, H-3\&4), 1.83-1.94 (2 H, m, H-2), 2.63-2.72 (2 H, m, H-5), 3.38$3.44(2 \mathrm{H}, \mathrm{t}, J=7.5 \mathrm{~Hz}, \mathrm{H}-1), 4.13-4.21(2 \mathrm{H}, \mathrm{q}, J=7.5 \mathrm{~Hz}, \mathrm{H}-9), 5.75-5.81(1 \mathrm{H}, \mathrm{dt}, J=2.5$ \& 
$12.5 \mathrm{~Hz}, \mathrm{H}-7), 6.15-6.26(1 \mathrm{H}, \mathrm{dt}, J=7.5 \& 12,5 \mathrm{~Hz}, \mathrm{H}-6) ;{ }^{13} \mathrm{C} \mathrm{NMR}\left(62.8 \mathrm{MHz}, \mathrm{CDCl}_{3}\right) 14.3$, 27.8, 28.1, 28.7, 32.5, 33.8, 59.9, 120.1, 149.9, 168.8; IR (cm-1) 2979 (s), 2932 (s), 2857 (s), $1714(\mathrm{~s}), 1643(\mathrm{~s}), 1444(\mathrm{~m}), 1414(\mathrm{~s}), 1386(\mathrm{~m}), 1364(\mathrm{w}), 1184(\mathrm{~s}), 1095(\mathrm{w}), 914(\mathrm{w}), 820(\mathrm{~m})$, 730 (w), 643 (w), 561 (w); HRMS required for $\left(\mathrm{C}_{10} \mathrm{H}_{18} \mathrm{BrO}_{2}\right)$ 249.0490; found $249.0485\left({ }^{79} \mathrm{Br}\right)$, $251.0464\left({ }^{81} \mathrm{Br}\right)$.

\section{(Z)-ethyl 8-nitrooct-2-enoate (19a)}

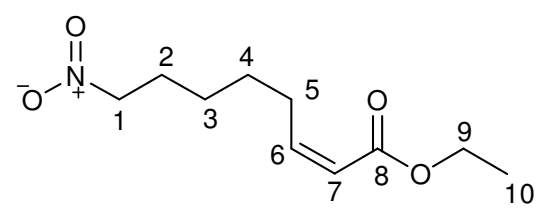

Following general procedure A, (Z)-ethyl 8-bromooct-2-enoate 18a $(0.74 \mathrm{~g}, 2.97 \mathrm{mmol})$ gave the crude product as a yellow oil, purification by column chromatography (silica gel, diethyl ether/hexane, 1/3) gave the title compound as a clear colourless oil $(0.32 \mathrm{~g}, 50 \%, 1.49 \mathrm{mmol})$.

${ }^{1} \mathrm{H}$ NMR (250 MHz, CDCl $) 1.26(3 \mathrm{H}, \mathrm{t}, J=7.5 \mathrm{~Hz}, \mathrm{H}-10), 1.37-1.63(4 \mathrm{H}, \mathrm{m}, \mathrm{H}-3 \& 4), 1.98-2.10$ (2 H, quin, $J=7.5 \mathrm{~Hz}, \mathrm{H}-2)$, 2.63-2.73 $(2 \mathrm{H}, \mathrm{dq}, J=2.5 \& 7.5 \mathrm{~Hz}, \mathrm{H}-5), 4.12-4.21(2 \mathrm{H}, \mathrm{q}, J=7.5$ $\mathrm{Hz}, \mathrm{H}-9), 4.36-4.42(2 \mathrm{H}, \mathrm{t}, J=7.5 \mathrm{~Hz}, \mathrm{H}-1), 5.76-5.82(1 \mathrm{H}, \mathrm{dt}, J=12.5$ \& $2.5 \mathrm{~Hz}, \mathrm{H}-7), 6.14-$ $6.24(1 \mathrm{H}, \mathrm{dt}, J=7.5 \& 10.0 \mathrm{~Hz}, \mathrm{H}-6) ;{ }^{13} \mathrm{C}$ NMR $\left(62.8 \mathrm{MHz}, \mathrm{CDCl}_{3}\right)$ 14.3, 25.8, 27.1, 28.1, 28.4, 59.9, 75.6, 120.4, 149.3, 166.8; IR ( $\left.\mathrm{cm}^{-1}\right) 2981(\mathrm{~m}), 2932$ (m), $2861(\mathrm{~m}), 2359$ (w), 2340 (w), $1715(\mathrm{~s}), 1643(\mathrm{~m}), 1549(\mathrm{~s}), 1435(\mathrm{~m}), 1415(\mathrm{~m}), 1384(\mathrm{~m}), 2289(\mathrm{w}), 1186(\mathrm{~s}), 1095(\mathrm{w}), 1034$ (m), $822(m), 737(m), 668(w), 615(w)$; HRMS required for $\left(\mathrm{C}_{10} \mathrm{H}_{17} \mathrm{NNaO}_{4}\right)$ 238.1055; found 238.1050.

\section{Horner-Wadsworth Olefination - General Procedure B}

To a suspension of $\mathrm{NaH}$ (1.2 eq.) in tetrahydrofuran $(0.2 \mathrm{M})$ at $0^{\circ} \mathrm{C}$ was added a solution of the phosphonoacetate (1.2 eq.) in tetrahydrofuran dropwise. This solution was left to stir for 15 minutes, or until effervescence ceased, before the addition of a solution of 6-bromo hexanal 17 (1 eq.) in tetrahydrofuran. This solution was left to warm to room temperature overnight. The reaction was then quenched with aqueous ammonium chloride, extracted with diethyl ether, the 
aqueous layer was washed with diethyl ether, and the combined organic fractions were dried over $\mathrm{MgSO}_{4}$, filtered and concentrated under reduced pressure to afford the crude olefination product.

(E)-Ethyl 2-benzyl-8-bromooct-2-enoate and (Z)-Ethyl 2-benzyl-8-bromooct-2-enoate (18b)

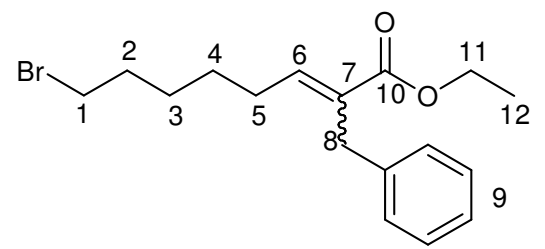

Following general procedure B ethyl 2-(diethoxyphosphoryl)-3-

phenylpropanoate $(2.5 \mathrm{~g}, 7.95 \mathrm{mmol})$ gave a crude mixture of products as a light yellow oil. Purification by column chromatography (silica gel, diethyl ether/hexane, 1/3) gave a mixture of the title compounds as a clear colourless oil (1.21 g, $46 \%, 3.62 \mathrm{mmol}) .{ }^{1} \mathrm{H}$ NMR (250 MHz, $\left.\mathrm{CDCl}_{3}\right)$ 1.21-1.31 (3 H, m), 1.40-1.49 (4 H, m), 1.79-1.94 (2 H, m), 2.25-2.51 (2 H m), 3.35-3.43 (2 H, m), 3.58-3.69 (2 H, d), 4.07-4.19 (2 H, m), 5.88-5.94 \& 6.88-6.94 (1 H, t, J=7.5 Hz \& $7.5 \mathrm{~Hz}) ;{ }^{13} \mathrm{C}$ NMR $(62.8 \mathrm{MHz}$, $\left.\mathrm{CDCl}_{3}\right)$ 14.2, 24.1, 27.6, 27.8, 27.9, 28.5, 28.7, 29.3, 32.4, 32.5, 33.6, 33.8, 34.1, 40.5, 60.2, $60.6,126.0,126.1,128.2,128.3,128.3,128.8,143.2,143.4$.

\section{(E)-Ethyl 2-benzyl-8-nitrooct-2-enoate and (Z)-Ethyl 2-benzyl-8-nitrooct-2-enoate (19b)}

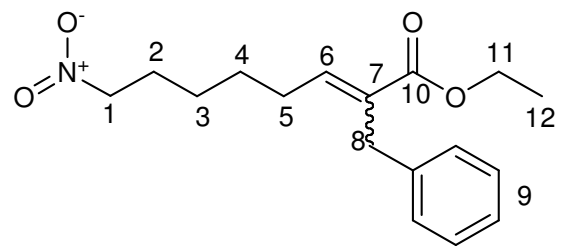

Following general procedure $\mathbf{A}$, a mixture of $(E)$-ethyl 2-benzyl-

8-bromooct-2-enoate and (Z)-ethyl 2-benzyl-8-bromooct-2enoate $18 \mathrm{~b}(1.21 \mathrm{~g}, 3.62 \mathrm{mmol})$ gave a crude mixture of products as a yellow oil. Purification by column chromatography (silica gel, diethyl ether/hexane, $1 / 3)$ gave pure title compounds as clear colourless oils. $(E)-18 b(0.16 \mathrm{~g}, 14 \%, 0.52 \mathrm{mmol})$ and (Z)-18b (0.22 g, $20 \%, 0.72 \mathrm{mmol}) .(E)-18 \mathbf{b}^{1} \mathrm{H}$ NMR (250 MHz, $\left.\mathrm{CDCl}_{3}\right)$ 1.20-1.26 (3 H, t, J = 7.5 $\mathrm{Hz}, \mathrm{H}-12), 1.31-1.56(4 \mathrm{H}, \mathrm{m}, \mathrm{H}-3$ \& 4), 1.91-2.02 (2 H, quin, J = 7.5 Hz, H-2), 2.24-2.33 (2 H, q, $J=\mathrm{H}-5), 3.68(2 \mathrm{H}, \mathrm{s}, \mathrm{H}-8), 4.11-4.19(2 \mathrm{H}, \mathrm{q}, J=7.5 \mathrm{~Hz}, \mathrm{H}-11), 4.30-4.36(2 \mathrm{H}, \mathrm{t}, J=7.5 \mathrm{~Hz}, \mathrm{H}-$ 1), 6.86-6.92 (1 H, t, J=7.5 Hz, H-6), 7.13-7.29 (5 H, m, H-9); $\left.{ }^{13} \mathrm{C} \mathrm{NMR} \mathrm{(62.8} \mathrm{MHz,} \mathrm{CDCl}_{3}\right)$ 14.1, $22.7,26.0,27.1,27.9,28.5,31.6,32.4,60.6,75.4,126.0,128.2,128.3,142.9 ; \mathrm{IR}\left(\mathrm{cm}^{-1}\right) 2979$ 
(m), $2933(\mathrm{~s}), 2860(\mathrm{~m}), 1707$ (s), $1644(\mathrm{~m}), 1600(\mathrm{~m}), 1551(\mathrm{~s}), 1494(\mathrm{~m}), 1452(\mathrm{~m}), 1382(\mathrm{~m})$, $1266(\mathrm{~m}), 1190$ (m), 1125 (s), 1078 (m), 910 (w), 736 (s), 699 (s), 614 (w); HRMS required for $\left(\mathrm{C}_{17} \mathrm{H}_{23} \mathrm{NaNO}_{4}\right)$ 328.1525; found 328.1515. (Z)-18b ${ }^{1} \mathrm{H} \mathrm{NMR}\left(250 \mathrm{MHz}, \mathrm{CDCl}_{3}\right)$ 1.16-1.22 (3 H, t, $J=7.5 \mathrm{~Hz}, \mathrm{H}-12), 1.33-1.54(4 \mathrm{H}, \mathrm{m}, \mathrm{H}-3$ \& 4), 1.94-2.05 (2 H, quin, $J=7.5 \mathrm{~Hz}, \mathrm{H}-2), 2.45-2.54$ $(2 \mathrm{H}, \mathrm{q}, J=\mathrm{H}-5), 3.58(2 \mathrm{H}, \mathrm{s}, \mathrm{H}-8), 4.07-4.15(2 \mathrm{H}, \mathrm{q}, J=7.5 \mathrm{~Hz}, \mathrm{H}-11), 4.32-4.38(2 \mathrm{H}, \mathrm{t}, J=$ 7.5 Hz, H-1), 5.85-5.91 (1 H, t, J = 7.5 Hz, H-6), 7.14-7.30 (5 H, m, H-9); ${ }^{13} \mathrm{C}$ NMR $(62.8 \mathrm{MHz}$, $\left.\mathrm{CDCl}_{3}\right)$ 14.1, 22.7, 26.0, 27.1, 27.9, 28.5, 31.6, 32.4, 60.6, 75.4, 126.0, 128.2, 128.3, 142.9; IR $\left(\mathrm{cm}^{-1}\right) 2979(\mathrm{~m}), 2929(\mathrm{~s}), 2859(\mathrm{~m}), 1711(\mathrm{~s}), 1642(\mathrm{~m}), 1602(\mathrm{~m}), 1551(\mathrm{~s}), 1494(\mathrm{~m}), 1452(\mathrm{~m})$, $1379(\mathrm{~m}), 1228(\mathrm{~m}), 1188(\mathrm{~m}), 1129(\mathrm{~s}), 1075(\mathrm{~m}), 910$ (w), 736 (s), 699 (s), $614(\mathrm{w})$; HRMS required for $\left(\mathrm{C}_{17} \mathrm{H}_{23} \mathrm{NaNO}_{4}\right) 328.1525$; found 328.1514.

\section{(Z)-Methyl 2-(N-tert-butoxycarbonyl)-amino-8-bromooct-2-enoate (18c)}

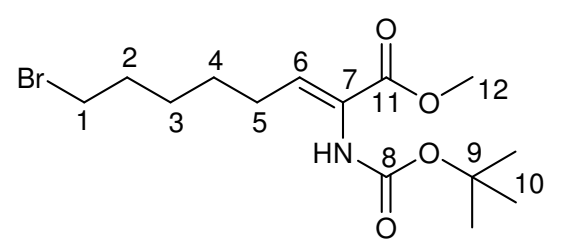

Following general procedure B methyl [(tertbutoxycarbonyl)amino] (dimethoxyphosphoryl)acetate (1.80 $\mathrm{g}$, $6.06 \mathrm{mmol}$ ) gave the crude product as a light yellow oil.

Purification by column chromatography (silica gel, diethyl ether/hexane, 3/7) gave the title product as a clear colourless oil $(0.70 \mathrm{~g}, 33 \%, 2.00 \mathrm{mmol}) .{ }^{1} \mathrm{H}$ NMR $\left(400 \mathrm{MHz}, \mathrm{CDCl}_{3}\right) 1.47$ (9 $\mathrm{H}, \mathrm{s}, \mathrm{H}-10), 1.47-1.50$ (4 H, m, H-3 \& 4), 1.85-1.88 (2 H, m, H-2), 2.54-2.56 (2 H, m, H-5), 3.39$3.41(2 \mathrm{H}, \mathrm{t}, \mathrm{J}=7.5 \mathrm{~Hz}, \mathrm{H}-1)$, $3.82(3 \mathrm{H}, \mathrm{s}, \mathrm{H}-12), 6.56(1 \mathrm{H}, \mathrm{br} \mathrm{s}, \mathrm{N}-H), 6.68(1 \mathrm{H}, \mathrm{br} \mathrm{s}, \mathrm{H}-6) ;{ }^{13} \mathrm{C}$ $\operatorname{NMR}\left(100 \mathrm{MHz}, \mathrm{CDCl}_{3}\right) 27.9,28.0,28.3,28.8,32.6,33.7,68.0,80.3,153.3,164.7 ; \mathrm{IR}\left(\mathrm{cm}^{-1}\right)$ $3416(\mathrm{~m}), 2976$ (s), 2932 (s), 2858 (m), 1724 (s), 1706 (s), 1511 (s), 1436 (s), 1367 (s), 1242 (s), $1161(\mathrm{~s}), 1048(\mathrm{~m}), 1021(\mathrm{~m}), 896(\mathrm{w}), 833(\mathrm{w}), 801(\mathrm{w}), 770(\mathrm{w}), 773(\mathrm{~m}), 645(\mathrm{w}), 561(\mathrm{~m})$; HRMS required for $\left(\mathrm{C}_{14} \mathrm{H}_{24} \mathrm{BrNNaO}_{4}\right) 372.0786$; found $372.0783\left({ }^{79} \mathrm{Br}\right)$ and $374.0762\left({ }^{81} \mathrm{Br}\right)$. 


\section{(Z)-Methyl 2-(N-tert-butoxycarbonyl)-amino-8-nitrooct-2-enoate (19c)}

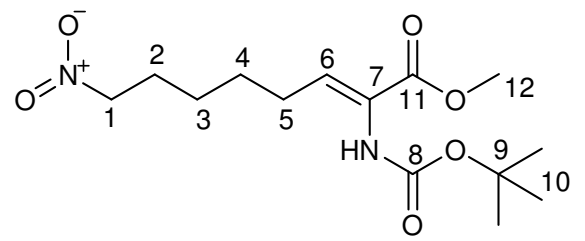

Following general procedure A, (Z)-methyl 2-(N-tertbutoxycarbonyl)-amino-8-bromooct-2-enoate $18 \mathrm{c}(0.70 \mathrm{~g}, 2.00$

mmol) gave the crude product as a yellow oil. Purification by

column chromatography (silica gel, diethyl ether/hexane, 2/3) gave the title compound as a clear colourless oil $(0.21 \mathrm{~g}, 34 \%, 0.67 \mathrm{mmol}) .{ }^{1} \mathrm{H}$ NMR $\left(400 \mathrm{MHz}, \mathrm{CDCl}_{3}\right) 1.40-1.50(4 \mathrm{H}, \mathrm{m}, \mathrm{H}-3$ \& 4), $1.48(9 \mathrm{H}, \mathrm{s}, \mathrm{H}-10), 2.00-2.04(2 \mathrm{H}, \mathrm{m}, \mathrm{H}-2), 2.21-2.26(2 \mathrm{H}, \mathrm{q}, \mathrm{J}=7.5 \mathrm{~Hz}, \mathrm{H}-5), 3.78(3 \mathrm{H}, \mathrm{s}, \mathrm{H}-$ 12), 4.37-4.41 (2 H, t, J= 7.5 Hz, H-1), $6.10(1 \mathrm{H}, \mathrm{br} \mathrm{s}, \mathrm{N}-H), 6.51-6.54(1 \mathrm{H}, \mathrm{t}, J=7.5 \mathrm{~Hz}, \mathrm{H}-6))$; ${ }^{13} \mathrm{C} \mathrm{NMR}\left(100 \mathrm{MHz}, \mathrm{CDCl}_{3}\right)$ 25.9, 27.0, 27.4, 27.8, 27.9, 28.1, 28.2, 52.3, 60.4, 75.5, 136.0, 165.3; IR (cm $\left.{ }^{-1}\right) 2978$ (m), 2933 (m), 1790 (w), 1726 (s), 1713 (s), 1658 (m), 1555 (s), 1494 (s), $1436(\mathrm{~m}), 1368$ (s), 1247 (s), 1163 (s), 1047 (m), 1026 (m), 914 (w), 842 (w), 775 (w); HRMS required for $\left(\mathrm{C}_{14} \mathrm{H}_{24} \mathrm{~N}_{2} \mathrm{NaO}_{6}\right) 339.1532$; found 339.1527.

\section{(Z)-Methyl 2-(N-benzylcarbonyl)-amino-8-bromooct-2-enoate (18d)}

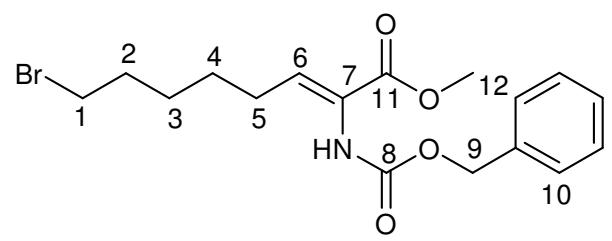

Following general procedure B methyl $\{[($ benzyloxy)carbonyl]amino $\}$ (dimethoxyphosphoryl)acetate

$(1.80 \mathrm{~g}, 5.43 \mathrm{mmol})$ gave the crude product as a light yellow oil. Purification by column chromatography (silica gel, diethyl ether/hexane, 3/7) gave the title compound as a clear colourless oil $(0.49 \mathrm{~g}, 24 \%, 1.28 \mathrm{mmol}) .{ }^{1} \mathrm{H} \mathrm{NMR}\left(400 \mathrm{MHz}, \mathrm{CDCl}_{3}\right) 1.47-$ $1.51(4 \mathrm{H}, \mathrm{m}, \mathrm{H}-3$ \& 4), 1.86-1.89 (2 H, m, H-2), 2.55-2.61 (2 H, m, H-5), 3.39-3.42 (2 H, t, J = $7.5 \mathrm{~Hz}, \mathrm{H}-1), 3.81(3 \mathrm{H}, \mathrm{s}, \mathrm{H}-12), 6.81-6.83\left(2 \mathrm{H}, \mathrm{br} \mathrm{s}, \mathrm{H}-6\right.$ \& N-H), 7.34-7.38 (5 H, m, H-10); ${ }^{13} \mathrm{C}$ NMR (100 MHz, $\left.\mathrm{CDCl}_{3}\right)$ 27.9, 28.0, 28.8, 32.6, 33.7, 52.3, 66.9, 128.2, 128.3, 128.6, 164.4; IR $\left(\mathrm{cm}^{-1}\right)$ 3405, (m), $2934(\mathrm{~s}), 2856(\mathrm{~m}), 1730(\mathrm{~s}), 1711(\mathrm{~s}), 1664(\mathrm{~m}), 1517(\mathrm{~s}), 1436(\mathrm{~s}), 1408(\mathrm{~m})$, $1333(\mathrm{~m}), 1216(\mathrm{~s}), 1043(\mathrm{~m}), 949(\mathrm{w}), 843(\mathrm{w}), 766(\mathrm{~m}), 739(\mathrm{~m}), 698(\mathrm{~m}), 642(\mathrm{w}), 593(\mathrm{w}), 560$ (w); HRMS required for $\left(\mathrm{C}_{17} \mathrm{H}_{22} \mathrm{BrNNaO}_{4}\right) 406.0630$; found $406.0626\left({ }^{79} \mathrm{Br}\right)$. 


\section{(Z)-Methyl 2-(N-benzylcarbonyl)-amino-8-nitrooct-2-enoate (19d)}

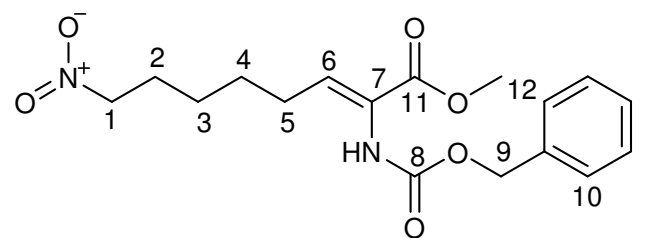

Following general procedure

A, (Z)-methyl 2-(Nbenzylcarbonyl)-amino-8-bromooct-2-enoate $18 \mathrm{~d}$ (0.49 g, $1.28 \mathrm{mmol}$ ) gave the crude product as a yellow oil.

Purification by column chromatography (silica gel, diethyl ether/hexane, 2/3) gave the title compound as a clear colourless oil $(0.19 \mathrm{~g}, 42 \%, 0.54 \mathrm{mmol}) .{ }^{1} \mathrm{H} \mathrm{NMR}\left(400 \mathrm{MHz}, \mathrm{CDCl}_{3}\right) 1.35-$ $1.42(2 \mathrm{H}, \mathrm{m}, \mathrm{H}-3), 1.47-1.54(2 \mathrm{H}, \mathrm{m}, \mathrm{H}-4), 1.94-2.01$ (2 H, m, H-2), 2.19-2.24 (2 H, m, H-5), $3.75(3 \mathrm{H}, \mathrm{s}, \mathrm{H}-12), 4.32-4.36(2 \mathrm{H}, \mathrm{t}, \mathrm{J}=7.5 \mathrm{~Hz}, \mathrm{H}-1), 5.14(2 \mathrm{H}, \mathrm{s}, \mathrm{H}-9), 6.31(1 \mathrm{H}, \mathrm{br} \mathrm{s}, \mathrm{N}-H)$, 6.58-6.61 (1 H, t, J = 7.5 Hz, H-6), 7.32-7.37 (5 H, m, H-10); ${ }^{13} \mathrm{C} \mathrm{NMR}\left(100 \mathrm{MHz}, \mathrm{CDCl}_{3}\right) 25.9$, $27.0,27.4,28.0,52.4,67.4,75.5,128.1,128.2,128.3,128.6,136.0,154.1,165.0 ; \mathrm{IR}\left(\mathrm{cm}^{-1}\right)$ 2951 (s), 2860 (m), 2253 (w), 1729 (s), 1709 (s), 1657 (m), 1553 (s), 1501 (s), 1454 (s), 1383 (m), 1231 (s), 1145 (w), 1108 (m), 1048 (s), 912 (m), 847 (w), 772 (m); HRMS required for $\left(\mathrm{C}_{17} \mathrm{H}_{22} \mathrm{~N}_{2} \mathrm{NaO}_{6}\right)$ 373.1376; found 373.1369.

\section{Synthesis of (E)-ethyl-5,5-dimethyl-8-nitrooct-2-enoate (25)}

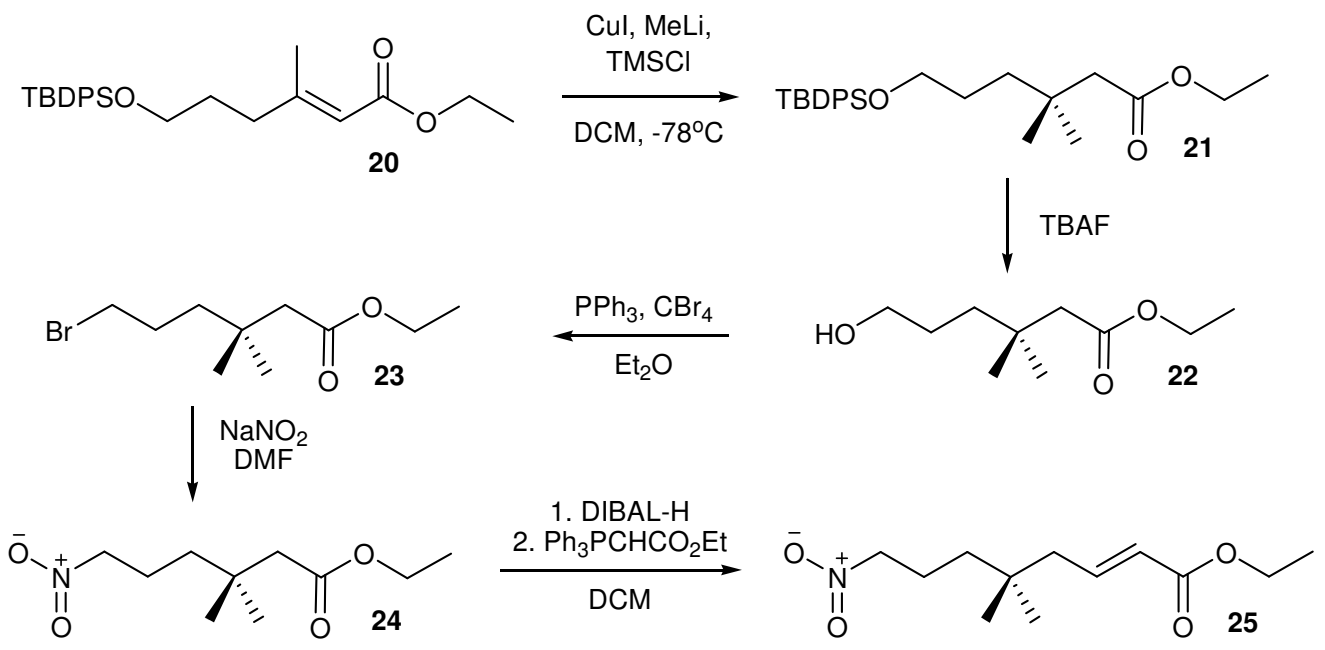


Ethyl 6-(tert-butyldiphenylsilyloxy)-3-methylhex-2-enoate (20) was synthesised following the procedure by Esumi T.; Zhao, M.; Kawakami, T.; Fukumoto, M.;
Toyota, M. \& Fukuyama, Y. Tetrahedron Lett., 2008, 2692.

\section{Ethyl 6-(tert-butyldiphenylsilyloxy)-3,3-dimethylhexanoate (21)}

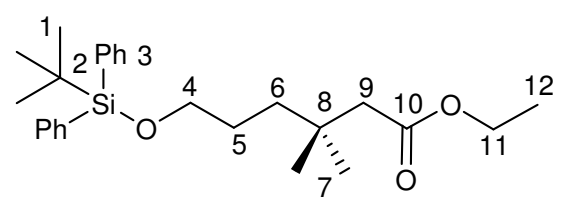

To a suspension of copper(l)iodide $(4.71 \mathrm{~g}, 24.74 \mathrm{mmol})$ in diethyl ether $(12.5 \mathrm{~mL})$ was added methyl lithium $(30.9 \mathrm{~mL} 49.49$ mmol) at $0{ }^{\circ} \mathrm{C}$ and the solution was stirred for 10 minutes. The

solvent was removed under reduced pressure at $0{ }^{\circ} \mathrm{C}$ and dichloromethane $(5 \mathrm{~mL})$ was added. The solution was stirred for $5 \mathrm{~min}$ at $0{ }^{\circ} \mathrm{C}$ and the solvent was removed under reduced pressure at $0{ }^{\circ} \mathrm{C}$. To the residue was added pre cooled dichloromethane $(150 \mathrm{~mL})$ and the mixture was cooled to $-78{ }^{\circ} \mathrm{C}$. To this cooled solution was added trimethyl silyl chloride $(3.1 \mathrm{~mL}, 24.74 \mathrm{mmol})$ drop-wise and ethyl 6-(tert-butyldiphenylsilyloxy)-3-methylhex-2-enoate 20 (5.08 g, $12.37 \mathrm{mmol}$ ) in dichloromethane $(25 \mathrm{~mL})$. The mixture was allowed to warm to $0{ }^{\circ} \mathrm{C}$ gradually and the reaction was quenched by the addition of a mixture of aqueous ammonium chloride and $28 \%$ ammonium hydroxide (1:1). The mixture was extracted with diethyl ether $(3 \times 100 \mathrm{~mL})$, the combined organic layers were dried over $\mathrm{Na}_{2} \mathrm{SO}_{4}$, filtered and concentrated under reduced pressure to afford the crude compound as a light yellow oil. Purification by column chromatography (silica gel, ethyl acetate/hexane, 1/9) gave the title compound as a clear colourless oil $(4.22 \mathrm{~g}, 80 \%$, $9.88 \mathrm{mmol}) .{ }^{1} \mathrm{H}$ NMR $\left(250 \mathrm{MHz}, \mathrm{CDCl}_{3}\right) 0.98(6 \mathrm{H}, \mathrm{s}, \mathrm{H}-7), 1.05(9 \mathrm{H}, \mathrm{s}, \mathrm{H}-1), 1.20-1.26(3 \mathrm{H}, \mathrm{t}, J$ $=7.5 \mathrm{~Hz}, \mathrm{H}-12), 1.27-1.36(2 \mathrm{H}, \mathrm{m}, \mathrm{H}-6), 1.49-1.58(2 \mathrm{H}, \mathrm{m}, \mathrm{H}-5), 2.17(2 \mathrm{H}, \mathrm{s}, \mathrm{H}-9), 3.61-3.67(2$ $\mathrm{H}, \mathrm{t}, J=7.5 \mathrm{~Hz}, \mathrm{H}-4), 4.05-4.14(2 \mathrm{H}, \mathrm{q}, J=7.5 \mathrm{~Hz}, \mathrm{H}-11), 7.34-7.42(6 \mathrm{H}, \mathrm{m}, \mathrm{H}-3), 7.65-7.68(4$ $\mathrm{H}, \mathrm{m}, \mathrm{H}-3) ;{ }^{13} \mathrm{C} \operatorname{NMR}\left(62.8 \mathrm{MHz}, \mathrm{CDCl}_{3}\right) 14.1,14.3,26.9,27.2,27.4,38.3,46.2,59.9,64.5$, 127.6, 129.5, 135.6; IR ( $\left.\mathrm{cm}^{-1}\right) 3069$ (w), 2957 (s), $2895(\mathrm{~m}), 1731(\mathrm{~s}), 1471(\mathrm{~m}), 1427(\mathrm{~m}), 1306$ 
(w), $1235(\mathrm{~m}), 1111(\mathrm{~s}), 1034(\mathrm{w}), 939$ (w), $823(\mathrm{~m}), 739(\mathrm{~m}), 702(\mathrm{~s}), 613(\mathrm{~m}), 504(\mathrm{~s})$; HRMS required for $\left(\mathrm{C}_{26} \mathrm{H}_{38} \mathrm{NaO}_{3} \mathrm{Si}\right) 449.2488$; found 449.2482 .

\section{Ethyl 6-hydroxy-3,3-dimethylhexanoate (22)}

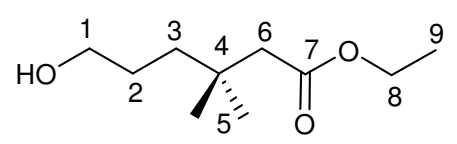

Ethyl 6-(tert-butyldiphenylsilyloxy)-3,3-dimethylhexanoate 21 (4.22 g,

$9.88 \mathrm{mmol})$ was dissolved in tetrabutyl ammonium fluoride $(12 \mathrm{~mL}$, $11.86 \mathrm{mmol}$ ) and stirred at room temperature overnight. This solution was concentrated under reduced pressure to give the crude product as a light yellow oil. Purification by chromatography (silica gel, ethyl acetate/hexane, 1/3) gave the title compound as a clear colourless oil (1.42 $\mathrm{g}$, $76 \%, 7.43 \mathrm{mmol}) .{ }^{1} \mathrm{H}$ NMR $\left(250 \mathrm{MHz}, \mathrm{CDCl}_{3}\right) 1.01(6 \mathrm{H}, \mathrm{s}, \mathrm{H}-5), 1.23-1.29(3 \mathrm{H}, \mathrm{t}, J=7.35 \mathrm{~Hz}$, H-9). 1.33-1.40 (2 H, m, H-3), 1.52-1.64 (3 H, m, H-2 \& O-H), $2.21(2 \mathrm{H}, \mathrm{s}, \mathrm{H}-6), 3.61-3.67(2 \mathrm{H}$, t, $J=7.5 \mathrm{~Hz}, \mathrm{H}-1), 4.08-4.16(2 \mathrm{H}, \mathrm{q}, J=7.5 \mathrm{~Hz}, \mathrm{H}-8) ;{ }^{13} \mathrm{C}$ NMR $\left(62.8 \mathrm{MHz}, \mathrm{CDCl}_{3}\right)$ 14.7, 27.8, 27.9, 33.5, 38.3, 46.3, 60.4, 63.9, 172.8; IR ( $\left.\mathrm{cm}^{-1}\right) 3400(\mathrm{~m}), 2958(\mathrm{~s}), 2872(\mathrm{~m}), 1731(\mathrm{~s}), 1466$ (m), $1368(\mathrm{~m}), 1308(\mathrm{~m}), 1231(\mathrm{~m}), 1124(\mathrm{~m}), 1056(\mathrm{~m}), 1031(\mathrm{~m})$; HRMS required for $\left(\mathrm{C}_{10} \mathrm{H}_{21} \mathrm{O}_{3}^{+}\right)$189.1483; found 189.1485 .

\section{Ethyl 6-bromo-3,3-dimethylhexanoate (23)}

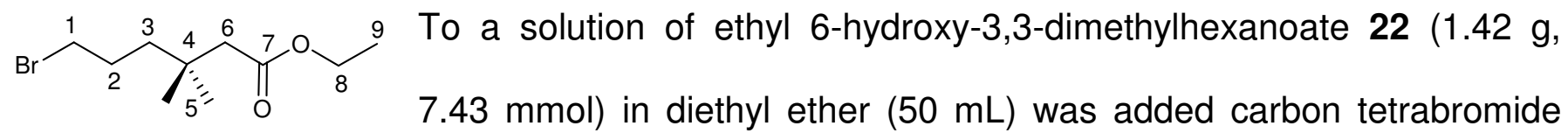
$(6.17 \mathrm{~g}, 18.59 \mathrm{mmol})$ and triphenyl phosphine $(5.07 \mathrm{~g}, 19.33 \mathrm{mmol})$. This solution was stirred for 1 hour, quenched with aqueous ammonium chloride, extracted with ether, the aqueous phase was washed with diethyl ether $(2 \times 15 \mathrm{~mL})$, the combined organic layers were dried over $\mathrm{MgSO}_{4}$, filtered and concentrated under reduced pressure to afford the crude compound as a light yellow oil. Purification by column chromatography (silica gel, diethyl ether/hexane, 1/3) gave the title compound as a clear colourless oil $(2.23 \mathrm{~g}, 94 \%, 7.00 \mathrm{mmol}) .{ }^{1} \mathrm{H} \mathrm{NMR}\left(250 \mathrm{MHz}, \mathrm{CDCl}_{3}\right) 1.01$ (6 H, s, H-5), 1.23-1.29 (3 H, t, J = 7.5 Hz, H-9), 1.41-1.48 (2 H, m, H-3), 1.81-1.93 (2 H, m, H- 
2), $2.19(2 \mathrm{H}, \mathrm{s}, \mathrm{H}-6)$, 3.36-3.42 (2 H, t, J = 7.5 Hz, H-1), 4.08-4.16 (2 H, q, J = 7.5 Hz, H-8); ${ }^{13} \mathrm{C}$ $\operatorname{NMR}\left(62.8 \mathrm{MHz}, \mathrm{CDCl}_{3}\right)$ 14.8, 27.8, 28.3, 33.5, 34.7, 40.9, 46.3, 60.4, 172.5; IR (cm $\left.{ }^{-1}\right) 2960$ (m), $1730(\mathrm{~s}), 1468(\mathrm{~m}), 1367(\mathrm{~m}), 1301(\mathrm{w}), 1228(\mathrm{~m}), 1133(\mathrm{~m}), 1037(\mathrm{~m}), 934(\mathrm{w}), 670(\mathrm{~m}), 560$ (w); HRMS required for $\left(\mathrm{C}_{10} \mathrm{H}_{19} \mathrm{BrNaO}_{2}\right)$ 273.0466; found $\left(\mathrm{Br}^{79}\right) 273.0473$ and $\left(\mathrm{Br}^{81}\right) 275.0452$.

\section{Ethyl 6-nitro-3,3-dimethylhexanoate (24)}

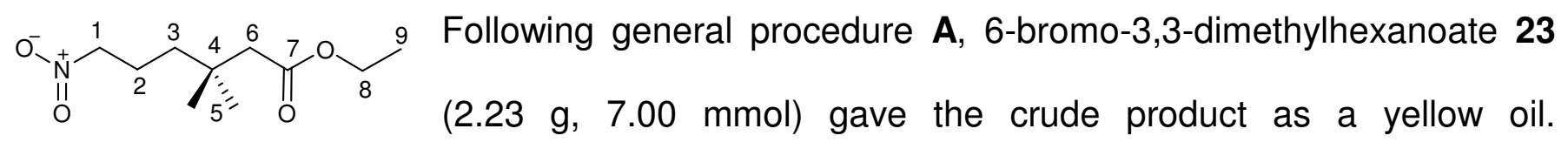

Purification by column chromatography (silica gel, diethyl ether/hexane, 1/3) gave the title compound as a clear colourless oil (0.55 g, $36 \%, 2.51 \mathrm{mmol}) .{ }^{1} \mathrm{H} \mathrm{NMR}\left(250 \mathrm{MHz}, \mathrm{CDCl}_{3}\right) 1.02$ (6 H, s, H-5), 1.23-1.29 (3 H, t, J=7.5 Hz, H-9), 1.36-1.44 (2 H, m, H-3), 1.97-2.09 (2 H, m, H2), $2.21(2 \mathrm{H}, \mathrm{s}, \mathrm{H}-6), 4.08-4.16(2 \mathrm{H}, \mathrm{q}, J=7.5 \mathrm{~Hz}, \mathrm{H}-8), 4.34-4.40(2 \mathrm{H}, \mathrm{t}, J=7.5 \mathrm{~Hz}, \mathrm{H}-1) ;{ }^{13} \mathrm{C}$ NMR $\left(62.8 \mathrm{MHz}, \mathrm{CDCl}_{3}\right)$ 14.7, 22.9, 27.7, 33.4, 38.3, 46.0, 60.6, 76.5; IR ( $\left.\mathrm{cm}^{-1}\right) 2963(\mathrm{~m}), 1727$ (s), $1575(\mathrm{~m}), 1552$ (s), 1466 (w), 1369 (m), 1333 (w), 1231 (m), 1125 (m), 1032 (m); HRMS required for $\left(\mathrm{C}_{10} \mathrm{H}_{19} \mathrm{NaNO}_{4}\right)$ 240.1212; found 240.1214.

\section{6-Nitro-3,3-dimethylhexanal}

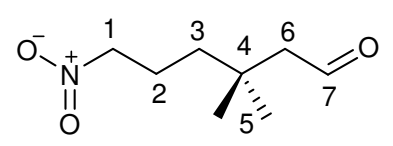

To a solution of ethyl 6-nitro-3,3-dimethylhexanoate $24(0.55 \mathrm{~g}, 2.51$ $\mathrm{mmol})$ in dichloromethane $(10 \mathrm{~mL})$ at $-78{ }^{\circ} \mathrm{C}$ was added DIBAL- $\mathrm{H}(2.8 \mathrm{~mL}$, $2.76 \mathrm{mmol}, 1 \mathrm{M}$ solution in hexanes). After 1 hour at $-78{ }^{\circ} \mathrm{C}$ TLC analysis showed consumption of starting material. The reaction was quenched with aqueous hydrochloric acid $(1 \mathrm{M}, 50 \mathrm{~mL})$ and water $(100 \mathrm{~mL})$, the aqueous layer was extracted with DCM $(2 \times 50 \mathrm{~mL})$. The combined organic layers were washed with water, dried over $\mathrm{MgSO}_{4}$, filtered and concentrated under reduced pressure to afford the crude product as a light yellow oil, which was used without further purification. ${ }^{1} \mathrm{H}$ NMR $\left(250 \mathrm{MHz}, \mathrm{CDCl}_{3}\right) 1.09(6 \mathrm{H}, \mathrm{s}, \mathrm{H}-5), 1.37-1.43(2 \mathrm{H}, \mathrm{m}, \mathrm{H}-3), 1.98-2.05(2$ 
$\mathrm{H}, \mathrm{m}, \mathrm{H}-2), 2.30-2.32(2 \mathrm{H}, \mathrm{d}, J=5.0 \mathrm{~Hz}, \mathrm{H}-6), 4.34-4.40(2 \mathrm{H}, \mathrm{t}, J=7.5 \mathrm{~Hz}, \mathrm{H}-1), 9.82-9.86$ (1 $\mathrm{H}, \mathrm{t}, J=5.0 \mathrm{~Hz}, \mathrm{H}-7)$.

\section{(E)-Ethyl 5,5-dimethyl-8-nitrooct-2-enoate (25)}

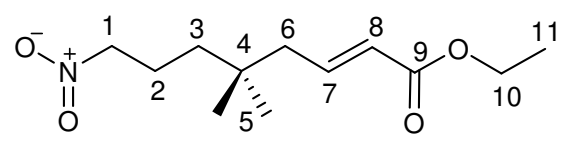

To a solution of crude 6-nitro-3,3-dimethylhexanal $(\sim 0.43 \mathrm{~g}$, $2.51 \mathrm{mmol})$ in dichloromethane $(15 \mathrm{~mL})$ was added in one portion (carbethoxymethylene)triphenylphosphorane $(1.00 \mathrm{~g}, 2.76 \mathrm{mmol})$. The reaction mixture was left to stir for 18 hours, quenched with saturated ammonium chloride $(10 \mathrm{~mL})$, the aqueous layer was extracted with dichloromethane $(2 \times 10 \mathrm{~mL})$. The combined organic layers were washed with water, dried over $\mathrm{MgSO}_{4}$, filtered and concentrated under reduced pressure to afford the crude product as a viscous yellow oil. Purification by column chromatography (silica gel, diethyl ether/hexane 1/3) afforded the title compound as a clear colourless oil $(0.32 \mathrm{~g}, 53 \%$, $1.33 \mathrm{mmol}) .{ }^{1} \mathrm{H}$ NMR $\left(250 \mathrm{MHz}, \mathrm{CDCl}_{3}\right) 0.94(6 \mathrm{H}, \mathrm{s}, \mathrm{H}-5), 1.24-1.33(5 \mathrm{H}, \mathrm{m}, \mathrm{H}-3$ \& 11), 1.93$2.05(2 \mathrm{H}, \mathrm{m}, \mathrm{H}-2), 2.10-2.14(2 \mathrm{H}, \mathrm{dd}, J=2.5 \& 10 \mathrm{~Hz}, \mathrm{H}-6), 4.15-4.24(2 \mathrm{H}, \mathrm{q}, J=7.5 \mathrm{~Hz}, \mathrm{H}-$ 10), 4.33-4.39 (2 H, t, J= 7.5 Hz, H-1), 5.79-5.86 (1 H, dt, J= 2.5 \& $15 \mathrm{~Hz}, \mathrm{H}-8), 6.87-7.00(1 \mathrm{H}$, app. quin, $J=7.5 \mathrm{~Hz}, \mathrm{H}-7) ;{ }^{13} \mathrm{C} \mathrm{NMR}\left(62.8 \mathrm{MHz}, \mathrm{CDCl}_{3}\right)$ 14.7, 22.8, 27.1, 34.0, 38.6, 45.0, 60.7, 76.4, 124.3, 145.8 ; IR ( $\left.\mathrm{cm}^{-1}\right) 2959$ (m), 2359 (s), 2340 (m), 1717 (s), 1653 (w), 1552 (s), 1465 (w), $1368(m), 1269(m), 1197(m), 1141(w), 1043(w), 986(w), 668(w)$; HRMS required for $\left(\mathrm{C}_{12} \mathrm{H}_{22} \mathrm{NO}_{4}^{+}\right)$244.1549; found 244.1543. 
Synthesis of (E)-ethyl 4-(3-nitropropoxy)but-2-enoate (E)-29

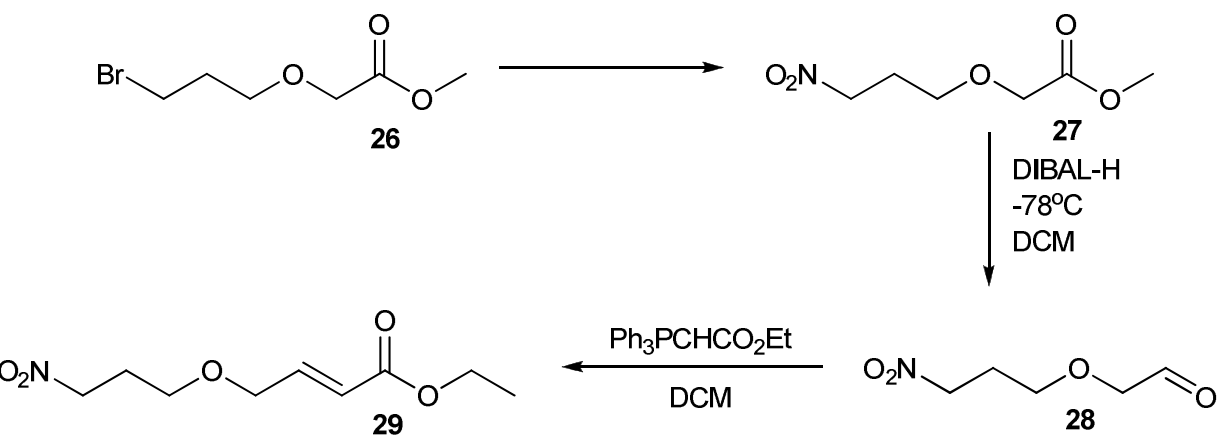

Methyl 6-bromo-3-oxahexanoate was synthesized according to
literature procedure. See Maruyama, T.; Asada, M.; Shiraishi, T.;

Egashira, H.; Yoshida, H.; Maruyama, T.; Ohuchida, S.; Nakai, H.; Hondo, K.; Toda, M. Bioorg. Med. Chem. 2002, 10, 975.

$\mathrm{O}_{2} \mathrm{~N} \underbrace{2}_{3} \underbrace{\mathrm{N}_{\mathrm{OMe}}}_{4} \begin{aligned} & \text { Methyl 6-nitro-3-oxahexanoate } 27 \text { was synthesized using general } \\ & \text { procedure A, where methyl 6-bromo-3-oxahexanoate } 26(0.976 \mathrm{~g}, 4.6\end{aligned}$ mmol) gave the title compound as a yellow oil which was purified by flash column chromatography (Ethyl Acetate : Hexanes, 3:7) to yield pure methyl 6-nitro-3-oxahexanoate 27 $(0.163 \mathrm{~g}, 18 \%)$ as a clear, colourless oil. ${ }^{1} \mathrm{H}$ NMR $\left(250 \mathrm{MHz}, \mathrm{CHCl}_{3}\right): 4.58(2 \mathrm{H}, \mathrm{t}, J=7.5, \mathrm{H}-1)$, $4.09(2 \mathrm{H}, \mathrm{s}, \mathrm{H}-4), 3.76(3 \mathrm{H}, \mathrm{s}, \mathrm{H}-5), 3.65(2 \mathrm{H}, \mathrm{t}, \mathrm{J}=5 \mathrm{~Hz}, \mathrm{H}-3), 2.31(2 \mathrm{H}, \mathrm{m}, \mathrm{H}-2) .{ }^{13} \mathrm{C}$ NMR $(62.5$ $\left.\mathrm{MHz}, \mathrm{CHCl}_{3}\right): 170.63,72.31,68.11,67.60,51.87,27.41$ HRMS expected for $\left(\mathrm{C}_{6} \mathrm{H}_{11} \mathrm{NNaO}_{5}\right)$ 200.0535 ; found 200.0525

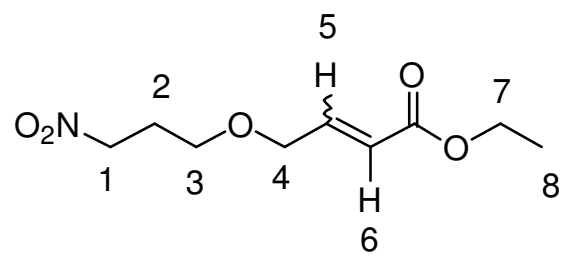

(E)-ethyl 4-(3-nitropropoxy)but-2-enoate $(E)$-29 and (Z)-ethyl 4-(3-nitropropoxy)but-2-enoate $(Z)-29$. To a solution of methyl 6-nitro-3-oxahexanoate $27(0.163 \mathrm{~g}, 0.85 \mathrm{mmol})$ in anhydrous 
DCM (25 mL) at $-78{ }^{\circ} \mathrm{C}$ was added DIBAL-H $(0.94 \mathrm{~mL}, 0.94 \mathrm{mmol}, 1 \mathrm{M}$ solution in hexanes). After 1 hour at $-78{ }^{\circ} \mathrm{C}$ TLC analysis showed consumption of starting material. The reaction was quenched with aqueous hydrochloric acid $(1 \mathrm{M}, 15 \mathrm{~mL})$ and water $(15 \mathrm{~mL})$, and the aqueous layer was extracted with dichloromethane $(2 \times 25 \mathrm{~mL})$. The combined organic layers were washed with water, dried over $\mathrm{MgSO}_{4}$, filtered and concentrated under reduced pressure to afford crude 2-(3-nitropropoxy)acetaldehyde $(0.120 \mathrm{~g})$. This product was used directly in the subsequent Wittig reaction. To a solution of crude 2-(3-nitropropoxy)acetaldehyde $(0.120 \mathrm{~g}, 0.8$ $\mathrm{mmol})$ in dichloromethane $(20 \mathrm{~mL})$ was added in one portion (carbmethoxymethylene)triphenylphosphorane $(0.322 \mathrm{~g}, 0.9 \mathrm{mmol})$. The reaction mixture was left to stir for 18 hours, quenched with saturated ammonium chloride $(10 \mathrm{~mL})$ and the aqueous layer was extracted with dichloromethane $(2 \times 15 \mathrm{~mL})$. The combined organic layers were washed with water, dried over $\mathrm{MgSO}_{4}$, filtered and concentrated under reduced pressure to afford a crude mixture of (E)-ethyl 4-(3-nitropropoxy)but-2-enoate and (Z)-ethyl 4-(3nitropropoxy)but-2-enoate as a yellow oil. The mixture was purified by flash column chromatography (Ethyl Acetate : Hexanes, $1: 3$ ) to give the pure title compounds as clear, colourless oils $(0.058 \mathrm{~g}$ and $0.033 \mathrm{~g}$ of $E$ - and $Z$ - respectively, $55 \%)$. ${ }^{1} \mathrm{H}$ NMR (E)-ethyl 4-(3nitropropoxy)but-2-enoate $(E)-29\left(250 \mathrm{MHz}, \mathrm{CHCl}_{3}\right): 6.93(1 \mathrm{H}, \mathrm{dt}, \mathrm{J}=5 \mathrm{~Hz} \& 17.5 \mathrm{~Hz}, \mathrm{H}-5), 6.03$ $(1 \mathrm{H}, \mathrm{dt}, J=2.5 \mathrm{~Hz}$ and $15 \mathrm{~Hz}, \mathrm{H}-6), 4.53(2 \mathrm{H}, \mathrm{t}, J=7.5 \mathrm{~Hz}, \mathrm{H}-4), 4.22(2 \mathrm{H}, \mathrm{q}, J=7.5 \mathrm{~Hz}, \mathrm{H}-7)$, $4.12(2 \mathrm{H}, \mathrm{m}, \mathrm{H}-3), 3.58(2 \mathrm{H}, \mathrm{t}, \mathrm{J}=7.5 \mathrm{~Hz}, \mathrm{H}-1), 2.30(2 \mathrm{H}, \mathrm{m}, \mathrm{H}-2), 1.28(3 \mathrm{H}, \mathrm{br}, \mathrm{H}-8) .{ }^{13} \mathrm{C}$ NMR (62.5 MHz, CHCl3) : 143.58, 121.47, 72.57, 69.63, 66.89, 60.51, 31.61, 22.68, 14.25. ${ }^{1} \mathrm{H}$ NMR (Z)-ethyl 4-(3-nitropropoxy)but-2-enoate (Z)-29 $\left(250 \mathrm{MHz}, \mathrm{CHCl}_{3}\right): 6.30(1 \mathrm{H}, \mathrm{m}, \mathrm{H}-5), 5.82(1 \mathrm{H}$, $\mathrm{dt}, J=2.5$ \& $12.5 \mathrm{~Hz}, \mathrm{H}-6), 4.55(4 \mathrm{H}, \mathrm{m}, \mathrm{H}-1$ and $\mathrm{H}-4), 4.17(2 \mathrm{H}, \mathrm{q}, J=7.5 \mathrm{~Hz}, \mathrm{H}-7), 2.28(2 \mathrm{H}$, m, H-2), $1.27(3 \mathrm{H}, \mathrm{t}, J=7.5 \mathrm{~Hz}, \mathrm{H}-8) .{ }^{13} \mathrm{C}$ NMR (62.5 MHz, CHCl3) : 147.52, 119.92, 72.70, 68.99, 66.66, 60.33, 27.54, 14.24. HRMS required for $\left(\mathrm{C}_{9} \mathrm{H}_{16} \mathrm{NO}_{5}{ }^{+}\right) 218.1028$; found 218.1019. 


\section{Racemic reaction}

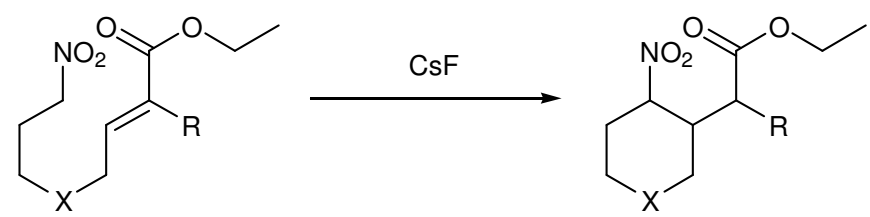

\section{General racemic reaction. General procedure C}

To a solution of the nitro- $\alpha, \beta$-unsaturated ester ( 1 eq.) in solvent ( $0.3 \mathrm{M}$ concentration), was added cesium fluoride (2 eq.) and the resulting solution stirred for $48 \mathrm{~h}$. The solution was concentrated under reduced pressure and purified by column chromatography.
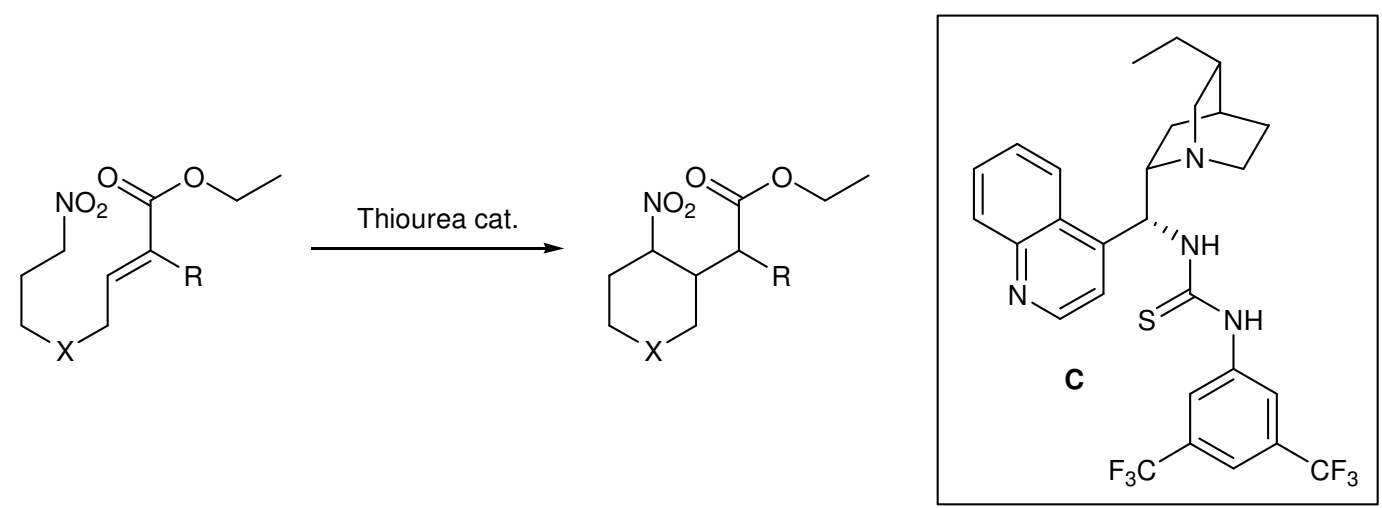

\section{General organocatalysis reaction. General procedure D}

To a solution of the nitro- $\alpha, \beta$-unsaturated ester (1 eq.) in solvent ( $0.5 \mathrm{M}$ concentration), was added bifunctional organocatalyst $\mathbf{C}(0.1$ eq.). The resulting solution was stirred for 7 days, concentrated under reduced pressure and purified by column chromatography.

\section{Ethyl 2-((1R,2S)-2-nitrocyclohexyl)acetate ((+)-2)}

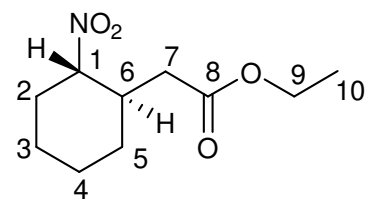

Following general procedure $\mathbf{D}$, using catalyst $\mathbf{C}$, in acetonitrile $(0.25 \mathrm{~mL})$, (E)-ethyl-8-nitrooct-2-enoate $1(161.5 \mathrm{mg}, 0.75 \mathrm{mmol})$ gave the crude product as a yellow oil. Purification by column chromatography (silica gel, diethyl ether/hexane, 1/3) gave the title product as a clear colourless oil $(140.0 \mathrm{mg}, 0.65 \mathrm{mmol}$, $87 \%)$. The enantiomeric excess was determined by HPLC analysis $\left(25^{\circ} \mathrm{C}\right)$ after DIBAL-H 
reduction to 30 and benzoyl protection to 32 to be $95.6 \%$. $[\alpha]_{D}^{23}+22.5\left(c=1.04, \mathrm{CHCl}_{3}\right) ;{ }^{1} \mathrm{H} \mathrm{NMR}$ (250 MHz, $\left.\mathrm{CDCl}_{3}\right)$ 1.13-1.45 (3 H, m, Сyc-H), 1.73-2.00 (4 H, m, Сус-H), 2.15-2.50 (4 H, m, H-7 \& Cyc-H), 4.09-4.17 (2 H, q, J = 7.5 Hz, H-9), 4.31-4.40 (1 H, dt, J = 2.5 \& $12.5 \mathrm{~Hz}, \mathrm{H}-1$ (transisomer(major))), 4.71-4.77 (cis-isomer(minor)); $\left.{ }^{13} \mathrm{C} \mathrm{NMR} \mathrm{(62.8} \mathrm{MHz,} \mathrm{CDCl}_{3}\right)$ 14.2, 21.4, 22.9, 24.3, 24.6, 27.3, 28.1, 30.1, 35.3, 35.5, 37.4, 37.8, 60.6, 60.7, 85.2, 89.6; IR (cm $\left.{ }^{-1}\right) 2939$ (s), $2863(\mathrm{~m}), 1731(\mathrm{~s}), 1547(\mathrm{~s}), 1450(\mathrm{~m}), 1370(\mathrm{~s}), 1349(\mathrm{~m}), 1291(\mathrm{~m}), 1244(\mathrm{~m}), 1223(\mathrm{~m}), 1176$ (s), $1112(\mathrm{~m}), 1028(\mathrm{~m}), 970(\mathrm{w}), 900(\mathrm{w}), 856(\mathrm{w}), 736(\mathrm{w})$; HRMS required for $\left(\mathrm{C}_{10} \mathrm{H}_{18} \mathrm{NO}_{4}{ }^{+}\right)$ 216.1236; found 216.1232.

\section{Methyl 2-((1R,2S)-2-nitrocyclohexyl)acetate ((+)-3)}

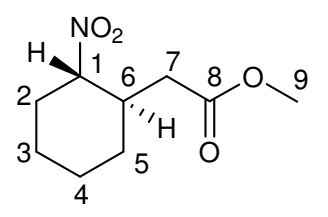

Following general procedure $\mathbf{D}$, using catalyst $\mathbf{C}$, in acetonitrile $(0.25 \mathrm{~mL}),(E)$ methyl 8-nitrooct-2-enoate $16 \mathrm{a}(161.5 \mathrm{mg}, 0.75 \mathrm{mmol})$ gave the crude product as a yellow oil. Purification by column chromatography (silica gel, diethyl ether/hexane, 1/3) gave the title compound as a clear colourless oil ( $85.5 \mathrm{mg}, 0.43 \mathrm{mmol}, 57 \%)$. The enantiomeric excess was determined by HPLC analysis $\left(25^{\circ} \mathrm{C}\right)$ after DIBAL-H reduction to 30 and benzoyl protection to 32 to be $96.4 \% .[\alpha]_{D}^{23}+21.5\left(c=1.01, \mathrm{CHCl}_{3}\right) ;{ }^{1} \mathrm{H} \mathrm{NMR}(250 \mathrm{MHz}$, $\left.\mathrm{CDCl}_{3}\right)$ 1.13-1.56 (3 H, m, Сyc-H), 1.74-1.99 (4 H, m, Сус-H), 2.16-2.45 (4 H, m, H-7 \& Cyc-H), $3.67(3 \mathrm{H}, \mathrm{s}, \mathrm{H}-9), 4.32-4.42\left(1 \mathrm{H}, \mathrm{dt}, \mathrm{J}=2.5\right.$ \& $15 \mathrm{~Hz}, \mathrm{H}-1$ (trans)), $4.71-4.77$ (Cis bridge); ${ }^{13} \mathrm{C}$ $\operatorname{NMR}\left(62.8 \mathrm{MHz}, \mathrm{CDCl}_{3}\right)$ 21.4, 22.8, 24.2, 24.6, 27.3, 28.1, 30.1, 30.3, 31.9, 35.0, 35.4, 37.1, 37.7, 51.7, 51.8, 85.2, 89.6, 172.0; IR ( $\left.\mathrm{cm}^{-1}\right) 2942(\mathrm{~s}), 2862(\mathrm{~m}), 1737(\mathrm{~s}), 1556(\mathrm{~s}), 1434(\mathrm{~m})$, $1354(m), 1292(m), 1245(m), 1170(m), 1112(m), 1004(m), 971(w), 914(w), 855(w), 735(s)$, 648 (w); HRMS required for $\left(\mathrm{C}_{9} \mathrm{H}_{15} \mathrm{NaNO}_{4}\right)$ 224.0899; found 224.0901. 


\section{Benzyl 2-((1R,2S)-2-nitrocyclohexyl)acetate ((+)-4)}

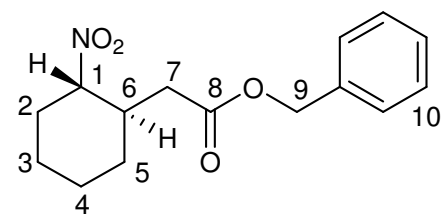

Following general procedure $\mathbf{D}$, using catalyst $\mathbf{C}$, in acetonitrile $(0.25$ $\mathrm{mL}),(E)$-benzyl 8-nitrooct-2-enoate 16b (161.5 mg, $0.75 \mathrm{mmol})$ gave the crude product as a yellow oil. Purification by column chromatography (silica gel, diethyl ether/hexane, 1/3) gave the title compound as a clear colourless oil ( $138.0 \mathrm{mg}, 0.50 \mathrm{mmol}, 66 \%$ ). The enantiomeric excess was determined by HPLC analysis $\left(25^{\circ} \mathrm{C}\right)$ after DIBAL-H reduction to $\mathbf{3 0}$ and benzoyl protection to $\mathbf{3 2}$ to be $98.0 \%$. $[\alpha]_{D}^{23}$ +20.5 $\left(c=0.91, \mathrm{CHCl}_{3}\right) ;{ }^{1} \mathrm{H}$ NMR $\left(250 \mathrm{MHz}, \mathrm{CDCl}_{3}\right)$ 1.05-1.96 $(7 \mathrm{H}, \mathrm{m}, \mathrm{Cyc}-\mathrm{H}), 2.19-2.29(2 \mathrm{H}$, m, Сус-H), 2.35-2.58 (2 H, m, Сyc-H), 4.29-4.40 (1 H, dt, J=5 \& $12.5 \mathrm{~Hz}, \mathrm{H}-1$ (trans ring bridge)), 4.68-4.74 (cis ring bridge), $5.10(2 \mathrm{H}, \mathrm{s}, \mathrm{H}-9), 7.25-7.39(5 \mathrm{H}, \mathrm{m}, \mathrm{H}-10) ;{ }^{13} \mathrm{C}$ NMR $(62.8$ $\left.\mathrm{MHz}, \mathrm{CDCl}_{3}\right) 14.1,21.4,22.7,22.8,24.2,24.5,27.2,28.0,30.1,30.3,31.6,31.8,35.2,35.4$, 37.3, 37.7, 66.4, 66.5, 85.1, 89.5, 128.2, 128.3, 128.3, 128.6; IR $\left(\mathrm{cm}^{-1}\right) 2940$ (s), 2861 (m), 2255 (w), $1736(\mathrm{~s}), 1547(\mathrm{~s}), 1454(\mathrm{~m}), 1415(\mathrm{w}), 1374(\mathrm{~m}), 1220(\mathrm{~m}), 1165(\mathrm{~s}), 1110(\mathrm{~m}), 1002(\mathrm{~m})$, 911 (s), 826 (w), 734 (s), 697 (m), $648(\mathrm{~m})$; HRMS required for $\left(\mathrm{C}_{15} \mathrm{H}_{19} \mathrm{NaNO}_{4}\right)$ 300.1212; found 300.1216 .

\section{Ethyl 2-((1R,2S)-5,5-dimethyl-2-nitrocyclohexyl)acetate ((+)-9)}

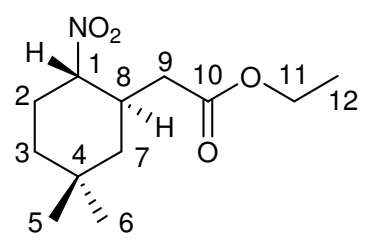

(silica gel, diethyl ether/hexane, 1/3) gave the title compound as a clear colourless oil $(92.6 \mathrm{mg}$, $0.38 \mathrm{mmol}, 76 \%$ ). The enantiomeric excess was determined by HPLC analysis $(25 \stackrel{\circ}{\circ} \mathrm{C})$ after DIBAL-H reduction to 31 and benzoyl protection to 33 to be $96.0 \%$. $[\alpha]_{D}^{23}+40.9(c=1.54$, $\left.\mathrm{CHCl}_{3}\right) ;{ }^{1} \mathrm{H} \mathrm{NMR}\left(250 \mathrm{MHz}, \mathrm{CDCl}_{3}\right) 0.96(3 \mathrm{H}, \mathrm{s}, \mathrm{H}-5$ or 6$), 1.05(3 \mathrm{H}, \mathrm{s}, \mathrm{H}-5$ or 6$), 0.97-1.15$ (1 $\mathrm{H}, \mathrm{m}$, Сус-H), 1.22-1.28 (3 H, t, J = 7.5 Hz, H-12), 1.26-1.36 (1 H, m, Сус-H), 1.52-1.73 (2 H, m, 
Сyc-H), 2.08-2.21 (3 H, m, H-9 \& Сyc-H), 2.32-2.39 (1 H, dd, J = 2.5 \& $15.0 \mathrm{~Hz}), 2.55-2.71(1 \mathrm{H}$, m, Cyc-H), 4.07-4.19 (2 H, m, H-11), 4.24-4.35 (1 H, m, H-1); ${ }^{13} \mathrm{C} N M R\left(62.8 \mathrm{MHz}, \mathrm{CDCl}_{3}\right)$ 14.2, 24.2, 24.5, 26.3, 28.1, 30.4, 32.1, 32.6, 33.9, 36.6, 36.9, 37.3, 39.3, 43.1, 43.3, 60.6, 90.0, 171.5; IR (cm $\left.{ }^{-1}\right) 2955$ (s), 1735 (s), 1546 (s), 1464 (m), 1371 (m), 1341 (m), 1251 (m), 1186 (s), $1142(\mathrm{~m}), 1095$ (w), 1034 (m), 910 (w), 873 (w), 756 (s), 734 (s); HRMS required for $\left(\mathrm{C}_{12} \mathrm{H}_{21} \mathrm{NaNO}_{4}\right) 266.1368$; found 266.1362.

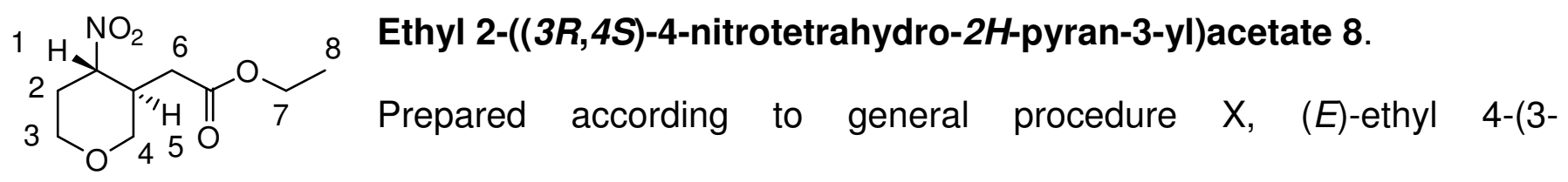
nitropropoxy)but-2-enoate $X(0.05 \mathrm{~g}, 0.23 \mathrm{mmol})$ gave the crude product as a yellow oil, which upon flash column chromatography (Ethyl acetate : Hexanes, $1: 4)$ afforded ethyl 2-((3R,4S)-4nitrotetrahydro-2H-pyran-3-yl)acetate 8 as a clear colourless oil $(0.034 \mathrm{~g}, 69 \%)$. The enantiomeric excess was determined by HPLC analysis $\left(25^{\circ} \mathrm{C}\right)$ to be $92 \% .{ }^{1} \mathrm{H}$ NMR $(400 \mathrm{MHz}$, $\left.\mathrm{CHCl}_{3}\right): 4.28(2 \mathrm{H}, \mathrm{m}, \mathrm{H}-6), 4.14(2 \mathrm{H}, \mathrm{m}, \mathrm{H}-7), 3.69(1 \mathrm{H}, \mathrm{dt}, J=2.5 \& 12.0 \mathrm{~Hz}, \mathrm{H}-5), 3.42(1 \mathrm{H}, \mathrm{t}$, $J=11 \mathrm{~Hz}, \mathrm{H}-1), 3.12(1 \mathrm{H}, \mathrm{m}$, ring-H), $2.72(2 \mathrm{H}, \mathrm{m}, \mathrm{H}-4), 2.41(1 \mathrm{H}, \mathrm{m}$, ring-H), $2.18(2 \mathrm{H}, \mathrm{dd}, J=$ 6.5 \& $16.9 \mathrm{~Hz}, \mathrm{H}-2), 1.28(3 \mathrm{H}, \mathrm{t}, \mathrm{J}=7.1 \mathrm{~Hz}, \mathrm{H}-8) .{ }^{13} \mathrm{C} \mathrm{NMR}\left(100 \mathrm{MHz}, \mathrm{CDCl}_{3}\right): 172.1,72.2$, 68.6, 60.8, 47.7, 42.4, 29.9, 14.2. HRMS required for $\left(\mathrm{C}_{9} \mathrm{H}_{16} \mathrm{NO}_{5}{ }^{+}\right) 218.1028$; found 218.1018

\section{(R)-Ethyl 2-((1R,2S)-2-nitrocyclohexyl)propanoate ((+)-5)}

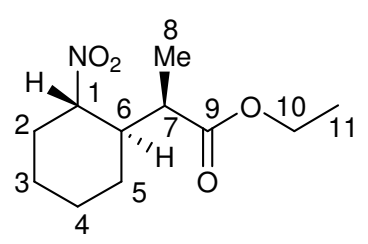

Following general procedure $\mathbf{D}$, using catalyst $\mathbf{C}$, in acetonitrile $(0.25 \mathrm{~mL})$, (E)-ethyl 2-methyl-8-nitrooct-2-enoate $16 \mathrm{c}(172.0 \mathrm{mg}, 0.75 \mathrm{mmol})$ gave the crude product as a yellow oil. Purification by column chromatography (silica

gel, diethyl ether/hexane, 1/3) gave the title compound as a clear colourless oil (91.7 mg, 0.40 mmol, $53 \%$. The enantiomeric excess was determined by HPLC analysis ( $\left.25{ }^{\circ} \mathrm{C}\right)$ to be $94 \%$. [Chiralcel OD column $(0.46 \mathrm{~cm} \varnothing \times 25 \mathrm{~cm})$ hexanes $/ 2$-propanol $=49 / 1$; flow rate $=0.3 \mathrm{~mL} / \mathrm{min}$; 
detection wavelength $=210 \mathrm{~nm}] \mathrm{tR} 19.56 \mathrm{~min}(R-1 S, 2 R) ; \mathrm{tR} 20.42 \mathrm{~min}(S-1 R, 2 S) .[\alpha]_{D}^{23}+14.3(c$ $\left.=0.95, \mathrm{CHCl}_{3}\right) ;{ }^{1} \mathrm{H} \mathrm{NMR}\left(250 \mathrm{MHz}, \mathrm{CDCl}_{3}\right) 1.10-1.13(3 \mathrm{H}, \mathrm{d}, \mathrm{J}=7.5 \mathrm{~Hz}, \mathrm{H}-8), 1.23-1.29(3 \mathrm{H}, \mathrm{t}$, $J=7.5 \mathrm{~Hz}, \mathrm{H}-11), 1.22-1.38(1 \mathrm{H}, \mathrm{m}$, Cyc-H), 1.63-2.03 (4 H, m, Сyc-H), 2.25-2.32 (1 H, m, CycH), 2.41-2.62 (2 H, m, H-7 \& Cyc-H), 4.10-4.19 (2 H, q, J = 7.5 Hz, H-10), 4.38-4.48 (1 H, dt, J= 2.5 \& $10 \mathrm{~Hz}, \mathrm{H}-1) ;{ }^{13} \mathrm{C} \mathrm{NMR}\left(62.8 \mathrm{MHz}, \mathrm{CDCl}_{3}\right)$ 9.8, 14.2, 14.7, 24.3, 24.5, 24.6, 25.0, 25.2, 32.0, 32.9, 39.4, 39.6, 42.4, 44.2, 60.5, 60.7, 87.8, 88.6, 174.5; IR $\left(\mathrm{cm}^{-1}\right) 2940$ (s), 2863 (s), $2339(\mathrm{w}), 1732$ (s), $1547(\mathrm{~s}), 1450(\mathrm{~m}), 1375(\mathrm{~m}), 1300(\mathrm{~m}), 1195(\mathrm{~s}), 1140$ (s0, $1054(\mathrm{~m}), 1020$ (w), 855 (w), $734(\mathrm{~m})$; HRMS required for $\left(\mathrm{C}_{11} \mathrm{H}_{19} \mathrm{NaNO}_{4}\right)$ 252.1212; found 252.1207.

\section{(R)-Ethyl 2-((1R,2S)-2-nitrocyclohexyl)-3-phenylpropanoate ((+)-6)}

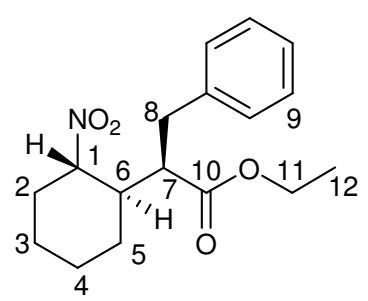

Following general procedure $\mathbf{D}$, using catalyst $\mathbf{C}$, in acetonitrile $(0.25 \mathrm{~mL})$, (E)-ethyl 2-benzyl-8-nitrooct-2-enoate $(E)-19 b(95.4 \mathrm{mg}, 0.31 \mathrm{mmol})$ gave the crude product as yellow oil. Purification by column chromatography (silica gel, diethyl ether/hexane, 1/3) gave the title compound as a clear colourless oil ( $50.0 \mathrm{mg}, 0.16 \mathrm{mmol}, 52 \%)$. The enantiomeric excess was determined by HPLC analysis $(25 \stackrel{\circ}{\circ})$ to be $>99 \%$. [Chiralcel OD column $(0.46 \mathrm{~cm} \varnothing \times 25 \mathrm{~cm})$ hexanes $/ 2-$ propanol $=$ 47.5/2.5; flow rate $=0.5 \mathrm{~mL} / \mathrm{min}$; detection wavelength $=210 \mathrm{~nm}] \mathrm{tR} 13.90 \mathrm{~min}(R-1 S, 2 R) ; \mathrm{tR}$ $15.40 \min (S-1 R, 2 S) \cdot[\alpha]_{D}^{23}+12.9\left(c=1.00, \mathrm{CHCl}_{3}\right) ;{ }^{1} \mathrm{H} \mathrm{NMR}\left(250 \mathrm{MHz}, \mathrm{CDCl}_{3}\right)$ 1.08-1.14 $(3 \mathrm{H}, \mathrm{t}$, $J=7.5 \mathrm{~Hz}, \mathrm{H}-12), 1.23-1.43(3 \mathrm{H}, \mathrm{m}$, Cyc-H), 1.79-2.05 (4 H, m, Сyc-H), 2.27-2.32 (1 H, m, СycH), 2.43-2.52 (1 H, m, Cyc-H), 2.58-2.65 (1 H, dt, $J=2.5 \& 12.5 \mathrm{~Hz}, \mathrm{H}-7), 2.72-2.79(1 \mathrm{H}, \mathrm{dd}, J=$ $2.5 \& 12.5 \mathrm{~Hz}, \mathrm{H}-8), 2.90-3.00(1 \mathrm{H}, \mathrm{dd}, J=12.5 \& 12.5 \mathrm{~Hz}, \mathrm{H}-8), 3.98-4.06(2 \mathrm{H}, \mathrm{q}, J=7.5 \mathrm{~Hz}$, $\mathrm{H}-11), 4.52-4.63(1 \mathrm{H}, \mathrm{dt}, J=5$ \& $12.5 \mathrm{~Hz}, \mathrm{H}-1), 7.13-7.28(5 \mathrm{H}, \mathrm{m}, \mathrm{H}-9) ;{ }^{13} \mathrm{C} N M R(62.8 \mathrm{MHz}$, $\left.\mathrm{CDCl}_{3}\right) 14.1,14.2,23.7,24.2,24.5,24.7,24.9,25.2,25.9,31.8,32.0,33.0,35.4,42.2,43.2$, 47.0, 48.6, 60.7, 87.9, 88.7, 126.4, 126.5, 128.4, 128.5, 128.9, 129.1; IR $\left(\mathrm{cm}^{-1}\right) 2939(\mathrm{~s}), 1729$ (s), 1602 (w), 1547 (s), $1494(\mathrm{~m}), 1453$ (m), 1370 (s), $1327(\mathrm{~m}), 1213$ (s), $1171(\mathrm{~s}), 1095$ (w), 
1031 (m), 903 (w), 860 (w), 753 (s); HRMS required for $\left(\mathrm{C}_{17} \mathrm{H}_{23} \mathrm{NaNO}_{4}\right)$ 328.1525; found 328.1520.

\section{(R)-Ethyl 2-(tert-butoxyaminocarbonyl)-2-((1R,2S)-2-nitrocyclohexyl)acetate ((-)-10)}

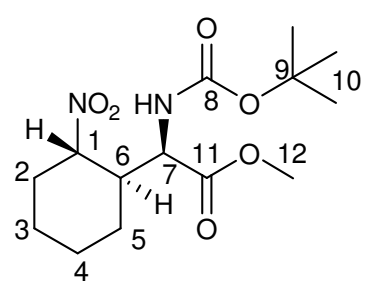

Following general procedure $\mathbf{D}$, using catalyst $\mathbf{C}$, in acetonitrile $(0.25 \mathrm{~mL})$,

(Z)-methyl 2-(N-tert-butoxycarbonyl)-amino-8-nitrooct-2-enoate $19 \mathrm{c}(50.0$ $\mathrm{mg}, 0.16 \mathrm{mmol}$ ) gave the crude product as a yellow oil. Purification by column chromatography (silica gel, diethyl ether/hexane, 3/7) gave the title compound as a clear colourless oil $(5.4 \mathrm{mg}, 0.02 \mathrm{mmol}, 11 \%)$. The enantiomeric excess was determined by HPLC analysis $(25 \stackrel{\circ}{\circ})$ to be $>99 \%$. [Chiralcel OD column $(0.46 \mathrm{~cm} \varnothing \times 25 \mathrm{~cm})$ hexanes $/ 2$-propanol $=47.5 / 2.5 ;$ flow rate $=0.5 \mathrm{~mL} / \mathrm{min}$; detection wavelength $=210 \mathrm{~nm}] \mathrm{tR}$ $29.56 \min (R-1 S, 2 R)$; tR $32.62 \min (S-1 R, 2 S) .[\alpha]_{D}^{23}-7.7\left(c=0.36, \mathrm{CHCl}_{3}\right) ;{ }^{1} \mathrm{H} \mathrm{NMR}(250 \mathrm{MHz}$, $\left.\mathrm{CDCl}_{3}\right)$ 1.18-1.36 (4 H, m, Сyc-H), 1.44.1.46 (9 H, m, H-10), 1.73-1.96 (3 H, m, Сyc-H), 2.26$2.70(2 \mathrm{H}, \mathrm{m}, \mathrm{Cyc}-\mathrm{H}), 3.76-3.79(3 \mathrm{H}, \mathrm{d}, \mathrm{J}=7.5 \mathrm{~Hz}, \mathrm{H}-12), 4.31-4.51(2 \mathrm{H}, \mathrm{m}, \mathrm{Cyc}-\mathrm{H})$, 5.05-5.22 $(1 \mathrm{H}, \mathrm{m}, \mathrm{Cyc}-\mathrm{H}) ;{ }^{13} \mathrm{C}$ NMR $\left(62.8 \mathrm{MHz}, \mathrm{CDCl}_{3}\right)$ 14.2, 24.1, 24.3, 24.4, 24.5, 24.4, 25.6, 26.9, 28.2, 28.3, 32.2, 33.0, 42.4, 52.6, 52.7, 55.5, 60.4, 80.4, 85.5, 86.2; IR ( $\left.\mathrm{cm}^{-1}\right) 3367(\mathrm{~m}), 2929(\mathrm{~m})$, $2358(\mathrm{~s}), 2339(\mathrm{~m}), 1714(\mathrm{~s}), 1549(\mathrm{~s}), 1502(\mathrm{w}), 1367(\mathrm{w}), 1261(\mathrm{w}), 1162(\mathrm{~m}), 1051(\mathrm{w}), 668$ (m); HRMS required for $\left(\mathrm{C}_{14} \mathrm{H}_{24} \mathrm{NaN}_{2} \mathrm{O}_{6}\right) 339.1532$; found 339.1528

\section{(R)-Methyl 2-(benzyloxyaminocarbonyl)-2-((1R,2S)-2-nitrocyclohexyl)acetate ((-)-7)}

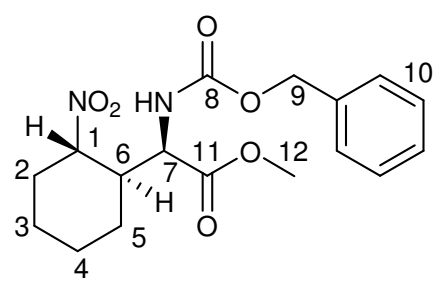

Following general procedure $\mathbf{D}$, using catalyst $\mathbf{C}$, (Z)-methyl 2-(Nbenzylcarbonyl)-amino-8-nitrooct-2-enoate $19 \mathrm{~d}(48.1 \mathrm{mg}, 0.14 \mathrm{mmol})$ gave the crude product as a yellow oil. Purification by column chromatography (silica gel, diethyl ether/hexane, 3/7) gave the title compound as a clear colourless oil ( $29.3 \mathrm{mg}, 0.08 \mathrm{mmol}, 61 \%)$. The enantiomeric excess was 
determined by HPLC analysis $\left(25^{\circ} \mathrm{C}\right)$ to be $95 \%$. [Chiralcel OD column $(0.46 \mathrm{~cm} ø \times 25 \mathrm{~cm})$ hexanes $/ 2$-propanol $=19 / 1$; flow rate $=1 \mathrm{~mL} / \mathrm{min}$; detection wavelength $=210 \mathrm{~nm}] \mathrm{tR} 30.74 \mathrm{~min}$ $(R-1 S, 2 R) ; \operatorname{tR} 44.29 \min (S-1 R, 2 S) .[\alpha]_{D}^{23}-6.0\left(c=0.98, \mathrm{CHCl}_{3}\right) ;{ }^{1} \mathrm{H} \mathrm{NMR}\left(250 \mathrm{MHz}, \mathrm{CDCl}_{3}\right)$ 1.20-1.21 (2 H, d, J = 2.5 Hz, Сус-H), 1.24-1.55 (3 H, m, Суc-H), 2.25-2.73 (2 H, m, Сус-H), 3.75-3.78 (3 H, m, J = 7.5 Hz, H-12), 4.37-4.61 (2 H, m, Cyc-H), 5.09-5.16 (2 H, m, H-9), 5.34$5.52(1 \mathrm{H}, \mathrm{m}, \mathrm{N}-H), 7.31-7.38(5 \mathrm{H}, \mathrm{m}, \mathrm{H}-10) ;{ }^{13} \mathrm{C} \mathrm{NMR}\left(62.8 \mathrm{MHz}, \mathrm{CDCl}_{3}\right)$ 24.1, 24.3, 24.4, 25.4, $32.1,33.0,42.5,43.3,52.7,52.9,55.8,67.4,67.5,85.6,86.1,128.3,128.3,128.6,136.0 ;$ IR $\left(\mathrm{cm}^{-1}\right) 3361(\mathrm{~m}), 2924(\mathrm{~m}), 2845(\mathrm{w}), 1724(\mathrm{~s}), 1547$ (s), $1524(\mathrm{~m}), 1339(\mathrm{w}), 1261$ (w), $1216(\mathrm{~m})$, $1068(\mathrm{~m}), 746(\mathrm{w})$; HRMS required for $\left(\mathrm{C}_{17} \mathrm{H}_{22} \mathrm{NaN}_{2} \mathrm{O}_{6}\right) 373.1376$; found 373.1371 .

\section{General reduction to alcohol procedure E}

To a solution of the nitro cyclohexyl ester (1 eq.) in dichloromethane $(0.06 \mathrm{M})$ at $-78^{\circ} \mathrm{C}$, was added DIBAL-H (4 eq., 1M solution in hexanes), the reaction was left to warm to room temperature overnight. The reaction was quenched with aqueous hydrochloric acid $(1 \mathrm{M})$ and water, the aqueous layer was extracted with dichloromethane. The combined organic layers were washed with water, dried over $\mathrm{MgSO}_{4}$, filtered and concentrated under reduced pressure to afford the nitro cyclohexyl alcohol, which was purified by column chromatography.

\section{2-((1R,2S)-2-nitrocyclohexyl)ethanol ((+)-30)}

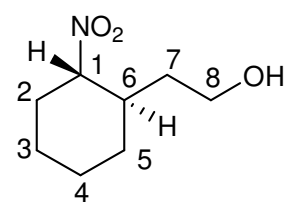

Following general procedure $\mathrm{E}$, ethyl 2-((1R,2S)-2-nitrocyclohexyl)acetate $((+)-$

2) $(85.5 \mathrm{mg}, 0.42 \mathrm{mmol})$ gave the crude product as a light yellow oil.

Purification by column chromatography (silica gel, ethyl acetate/hexane, 2/3)

afforded the title compound as white crystalline needles $(57.4 \mathrm{mg}, 79 \%, 0.33 \mathrm{mmol}) \cdot[\alpha]_{D}^{23}+26.8$ $\left(c=0.94, \mathrm{CHCl}_{3}\right) ; \mathrm{MP}-59.1-59.6{ }^{\circ} \mathrm{C} ;{ }^{1} \mathrm{H} \mathrm{NMR}\left(250 \mathrm{MHz}, \mathrm{CDCl}_{3}\right)$ 0.99-1.15 $(1 \mathrm{H}, \mathrm{m}, \mathrm{Cyc}-\mathrm{H})$, 1.23-1.50 (3 H, m, H-7 \& Сус-H), 1.54-1.67 (1 H, m, H-7), 1.73-2.27 (6 H, m, Сус-H), 3.58-3.75 
$(2 \mathrm{H}, \mathrm{m}, \mathrm{H}-8), 4.23-4.33(1 \mathrm{H}, \mathrm{dt}, J=5.0 \& 12.5 \mathrm{~Hz}, \mathrm{H}-1), ;{ }^{13} \mathrm{C} \mathrm{NMR}\left(62.8 \mathrm{MHz}, \mathrm{CDCl}_{3}\right) 24.3$,

24.7, 29.8, 32.1, 35.4, 37.8, 59.6, 91.3; IR ( $\left.\mathrm{cm}^{-1}\right) 3302(\mathrm{~m}), 2938(\mathrm{~m}), 2863(\mathrm{~m}), 1545(\mathrm{~s}), 1451$

(m), 1366 (s), 1296 (w), 1248 (w), $1197(w), 1090$ (w), $1048(m), 1005(m), 850(w), 733(w), 670$

(w); HRMS required for $\left(\mathrm{C}_{8} \mathrm{H}_{16} \mathrm{O}_{3} \mathrm{~N}^{+}\right)$174.1130; found 174.1123 .

\section{2-((1R,2S)-5,5-dimethyl-2-nitrocyclohexyl)ethanol ((+)-31)}

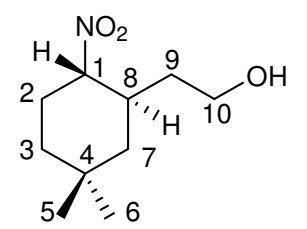

Following general procedure E, ethyl 2-((1R,2S)-5,5-dimethyl-2nitrocyclohexyl)acetate ((+)-9) $(92.6 \mathrm{mg}, 0.38 \mathrm{mmol})$ gave the crude product as a light yellow oil. Purification by column chromatography (silica gel, ethyl acetate/hexane, 2/3) afforded the title compound as a clear colourless oil $(73.1 \mathrm{mg}, 95 \%, 0.36$ mmol). $[\alpha]_{D}^{23}+26.6\left(c=0.98, \mathrm{CHCl}_{3}\right) ;{ }^{1} \mathrm{H} \mathrm{NMR}\left(250 \mathrm{MHz}, \mathrm{CDCl}_{3}\right) 0.96(3 \mathrm{H}, \mathrm{s}, \mathrm{H}-5$ or 6$), 1.03(3$ $\mathrm{H}, \mathrm{s}, \mathrm{H}-5$ or H-6), 1.00-1.09 (1 H, m, Сyc-H), 1.16-1.47 (3 H, m, H-9 \& Cyc-H), 1.50-1.68 (3 H, m, H-9 \& Суc-H), $1.88(1 \mathrm{H}$, br s, O-H), 2.05-2.12 (2 H, m, H-8 \& Суc-H), 2.26-2.42 (1 H, m, Cyc-H), 3.56-3.73 (2 H, m, H-10), 4.11-4.26 (1 H, m, H-1); ${ }^{13} \mathrm{C} \mathrm{NMR}\left(62.8 \mathrm{MHz}, \mathrm{CDCl}_{3}\right) 24.4$, 28.3, 32.3, 33.5, 35.7, 36. 9, 43.0, 59.6, 91.7; IR $\left(\mathrm{cm}^{-1}\right) 3369(\mathrm{~m}), 2949$ (s), 1545 (s), 1457 (m), $1373(\mathrm{~m}), 1266(\mathrm{w}), 1173(\mathrm{w}), 1045(\mathrm{~m}), 865(\mathrm{w}), 751(\mathrm{w})$; HRMS required for $\left(\mathrm{C}_{10} \mathrm{H}_{19} \mathrm{NaNO}_{3}\right)$ 224.1263; found 224.1256.

\section{General benzoyl protection procedure F}

To a solution of the nitro cyclohexyl alcohol (1 eq.) in dichloromethane $(0.03 \mathrm{M})$ was added benzoyl chloride (1.5 eq.) and pyridine (1.5 eq.). The solution was stirred for 1 hour and quenched with aqueous ammonium chloride. The aqueous layer was washed with dichloromethane; the combined organic layers were washed with water, dried over $\mathrm{MgSO}_{4}$, filtered and concentrated under reduced pressure to afford the benzoate, which was purified by column chromatography. 


\section{2-((1R,2S)-2-nitrocyclohexyl)ethyl benzoate ((+)-32)}

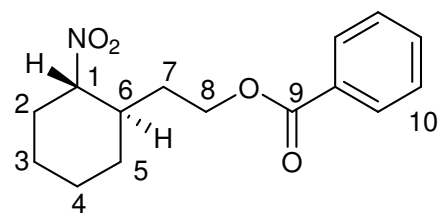

Following general procedure $\mathbf{F}, 2-((1 R, 2 S)-2$-nitrocyclohexyl)ethanol

$((+)-30)(71.3 \mathrm{mg}, 0.41 \mathrm{mmol})$ gave the crude product as a light yellow oil. Purification by column chromatography (silica gel, diethyl ether/hexane, 1/4) afforded the title product as a clear colourless oil $(88.7 \mathrm{mg}, 78 \%, 0.32$ mmol). Enantiomeric excess was determined by HPLC analysis $\left(25^{\circ} \mathrm{C}\right)$. [Chiralpak AD-H column $(0.46 \mathrm{~cm} \varnothing \mathrm{X} 25 \mathrm{~cm})$ hexanes $/ 2$-propanol $=19 / 1$; flow rate $=0.4 \mathrm{~mL} / \mathrm{min}$; detection wavelength $=210 \mathrm{~nm}] \mathrm{tR} 30.76 \mathrm{~min}(1 R, 2 S)$; tR $33.66 \mathrm{~min}(1 S, 2 R) . \quad[\alpha]_{D}^{23}+8.9\left(c=2.6, \mathrm{CHCl}_{3}\right)$; NMR (250 MHz, $\left.\mathrm{CDCl}_{3}\right)$ 1.03-1.19 (1 H, m, Cyc-H), 1.25-1.49 (3 H, m, Сyc-H), 1.55-1.97 (6 H, m, H-7 \& Сус-H), 2.05-2.30 (3 H, m, H-6 \& Cyc-H), 4.25-4.46 (3 H, m, H-1 \& H-8), 7.42-7.51 (3 $\mathrm{H}, \mathrm{m}, \mathrm{H}-10)$, 8.01-8.06 (2 H, m, H-10); ${ }^{13} \mathrm{C}$ NMR (62.8 MHz, $\left.\mathrm{CDCl}_{3}\right)$ 22.0, 22.2, 24.3, 24.7, 27.0, $27.4,28.4,29.7,31.5,32.1,36.0,38.2,61.9,62.6,86.1,91.0,128.5,128.5,128.9,129.6,130.2$, 130.6; IR ( $\left.\mathrm{cm}^{-1}\right) 2938(\mathrm{~m}), 2860$ (w), 1718 (s), 1545 (s), $1450(\mathrm{~m}), 1374(\mathrm{w}), 1315(\mathrm{~m}), 1274(\mathrm{~s})$, 1175 (w), 1113 (m), 1069 (w), 1025 (w), 712 (s); HRMS required for $\left(\mathrm{C}_{15} \mathrm{H}_{19} \mathrm{NNaO}_{4}\right)$ 300.1212; found 300.1206 .

\section{2-((1R,2S)-5,5-dimethyl-2-nitrocyclohexyl)ethyl benzoate ((+)-33)}

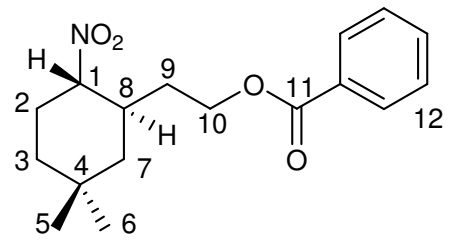

Following general procedure $\mathbf{F}, \quad 2-((1 R, 2 S)-5,5-$ dimethyl-2nitrocyclohexyl)ethanol $((+)-31)(73.1 \mathrm{mg}, 0.36 \mathrm{mmol})$ gave the crude product as a light yellow oil. Purification by column chromatography (silica gel, diethyl ether/hexane, 1/4) afforded the title compound as a clear colourless oil (88.6 $\mathrm{mg}, 82 \%, 0.29 \mathrm{mmol})$. Enantiomeric excess was determined by HPLC analysis (25 $\left.{ }^{\circ} \mathrm{C}\right)$. [Chiralpak AD-H column $(0.46 \mathrm{~cm} ø X 25 \mathrm{~cm})$ hexanes $/ 2$-propanol $=24 / 1$; flow rate $=0.5$ $\mathrm{mL} / \mathrm{min}$; detection wavelength $=210 \mathrm{~nm}] \mathrm{tR} 23.10 \mathrm{~min}(1 R, 2 S) ; \mathrm{tR} 24.23 \mathrm{~min}(1 S, 2 R) . \quad[\alpha]_{D}^{23}$ +6.1 $\left(c=1.2, \mathrm{CHCl}_{3}\right)$; NMR $\left(400 \mathrm{MHz}, \mathrm{CDCl}_{3}\right) 0.96(3 \mathrm{H}, \mathrm{s}, \mathrm{H}-5$ or 6$), 1.00(3 \mathrm{H}, \mathrm{s}, \mathrm{H}-5$ or H-6), 
1.03-1.06 (1 H, m, Сyc-H), 1.24-1.32 (1 H, m, Сyc-H), 1.50-1.62 (2 H, m, H-8 \& H-9), 1.68-1.73 (1 H, m, Сус-H), 1.83-1.87 (1 H, m, H-9), 2.07-2.13 (2 H, m, Сус-H), 2.35-2.43 (1 H, m, Сус-H), 4.17-4.24 (1 H, m, H-1), 4.28-4.36 (2 H, m, H-10), 7.41-7.47 (3 H, m, H-12), 8.02-8.05 (2 H, m, $\mathrm{H}-12) ;{ }^{13} \mathrm{C}$ NMR (100 MHz, $\mathrm{CDCl}_{3}$ ) 24.3, 28.3, 30.3, 31.5, 32.2, 34.1, 36.9, 42.8, 61.8, 91.4, 128.4, 128.5, 128.9, 130.2, 130.6, 162.4; IR ( $\left.\mathrm{cm}^{-1}\right) 2952$ (w), 2360 (w), 1785 (s), 1718 (s), 1686 (s), 1598 (w), $1544(m), 1451(m), 1423(w), 1324$ (w), 1275 (s), $1212(s), 1172(m), 1072(w)$, 1039 (s), 1016 (s), 995 (s), 777 (w), 706 (s), 615 (w); HRMS required for $\left(\mathrm{C}_{17} \mathrm{H}_{23} \mathrm{NNaO}_{4}\right)$ 328.1525; found 328.1521.

\section{Absolute configuration determined by Nef reaction and comparison of products optical rotation with literature value.}

\section{(R)-Ethyl 2-(2-oxocyclohexyl)acetate ((+)-34)}

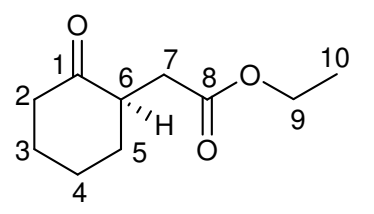

To a solution of ethyl 2-((1R,2S)-2-nitrocyclohexyl)acetate $((+)-2)(0.11 \mathrm{~g}$, $0.53 \mathrm{mmol})$ in ethanol $(2.5 \mathrm{~mL})$ was added sodium ethoxide $(0.14 \mathrm{~g}, 2.12$ $\mathrm{mmol})$. This solution was stirred for one hour before the addition of a solution of aqueous hydrochloric acid/ethanol (1:1(1M, $5.3 \mathrm{~mL}$ : ethanol $5.3 \mathrm{~mL}))$, after a further 10 minutes of stirring the reaction was diluted with water $(5 \mathrm{~mL})$ and extracted with diethyl ether (2 $\times 10 \mathrm{~mL})$. The combined organic layers were washed with water, dried over $\mathrm{Na}_{2} \mathrm{SO}_{4}$, filtered and concentrated under reduced pressure to afford the crude product as a light yellow oil. Purification by column chromatography (silica gel, ethyl acetate/hexane, 1/9) afforded the title compound as a clear colourless oil $(42.0 \mathrm{mg}, 43 \%, 0.23 \mathrm{mmol}) .[\alpha]_{D}^{23}+17.5(c=1.00, \mathrm{MeOH})$ (lit. -7.0 [(S)-ethyl 2-(2-oxocyclohexyl)acetate] Hiroi, K.; Achiwa, K.; Yamada, S.; Chem. Pharm. Bull.; 1972; 20; 246), ${ }^{1} \mathrm{H}$ NMR (250 MHz, $\left.\mathrm{CDCl}_{3}\right)$ 1.23-1.29 (3 H, t, $\left.J=7.5 \mathrm{~Hz}, \mathrm{H}-10\right), 1.33-1.50$ (1 H, m, Сус-H), 1.64-1.93 (3 H, m, Сус-H), 2.08-2.19 (3 H, m, H-7 \& Сус-H), 2.35-2.42 (2 H, m, 
Cyc-H), 2.72-2.94 (2 H, m, H-6 \& H-7), 4.09-4.18 (2 H, q, J = 7.5 Hz, H-9); ${ }^{13} \mathrm{C} N M R(62.8 \mathrm{MHz}$, $\left.\mathrm{CDCl}_{3}\right)$ 14.6, 25.6, 28.2, 34.3, 34.9, 42.2, 47.5, 60.8, 173.0, 211.5; IR ( $\left.\mathrm{cm}^{-1}\right) 2933(\mathrm{~m}), 2860(\mathrm{w})$, 1733 (s), $1711(s), 1448$ (w), 1367 (w), 1346 (w), 1276 (w), 1165 (m), 1130 (w), 1028 (w); HRMS required for $\left(\mathrm{C}_{10} \mathrm{H}_{17} \mathrm{O}_{3}\right)$ 185.1178; found 185.1172 .

\section{General Raney nickel cyclisation procedure $\mathbf{F}$}

To a solution of the nitro compound in ethanol $(0.1 \mathrm{M})$ was added Raney Nickel (1 Spatula), the solution was placed under an atmosphere of hydrogen (1 atm.) after flushing the system with argon until TLC showed the reaction was complete. Toluene (Same volume as ethanol) was added to the solution and heated to complete cyclisation. After TLC showed the reaction had gone to completion, the mixture was filtered through a pad of Celite, concentrated under reduced pressure and purified by column chromatography.

\section{(3S,3aR,7aS)-3-methyl-hexahydro-1H-indol-2(3H)-one, racemic (( \pm -35)}

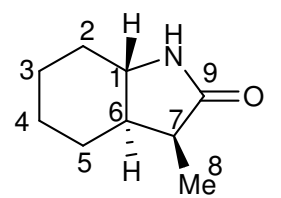

Following general procedure $\mathbf{F}$, racemic ethyl 2-(2-nitrocyclohexyl)propanoate $(( \pm)-5)(121.0 \mathrm{mg}, 0.53 \mathrm{mmol})$, gave the crude product which was purified by column chromatography (silica gel, ethyl acetate/hexane, 2/3) afforded the title compound as a white crystalline solid (26.8 $\mathrm{mg}, 0.17 \mathrm{mmol}, 33 \%)$. MP - 91.1-91.8 ${ }^{\circ} \mathrm{C} ;{ }^{1} \mathrm{H}$ NMR (400 MHz, $\left.\mathrm{CDCl}_{3}\right)$ 1.06-1.08 (3 H, d, J = 7.5 Hz, H-8), 1.13-1.17 (1 H, m, Cyc-H), 1.25-1.35 (3 H, m, Сус-H), 1.65-1.70 (1 H, m, Сус-H), 1.78-1.91 (2 H, m, Сус-H), 2.03-2.12 (2 H, m, Сус-H), 2.35-2.42 (1 H, m, H-7), 3.16-3.23 (1 H, dt, $J=5.0$ \& $12.5 \mathrm{~Hz}), 6.35(1 \mathrm{H}, \mathrm{br} \mathrm{s}, \mathrm{N}-H) ;{ }^{13} \mathrm{C}$ NMR $\left(100 \mathrm{MHz}, \mathrm{CDCl}_{3}\right)$ 9.7, 24.4, 24.8, 25.7, 31.2, 40.5, 47.9, 57.4, 182.2; IR (cm $\left.{ }^{-1}\right) 3245$ (w), 2930 (m), 2856 (w), 1696 (s), 1449 (w), 1376 (w), 1270 (w), 1101 (w), 755 (w); HRMS required for $\left(\mathrm{C}_{9} \mathrm{H}_{16} \mathrm{NO}^{+}\right)$154.1226; found 154.1223. 
(3R,3aR,7aS)-3-methyl-hexahydro-1H-indol-2(3H)-one, chiral ((+)-35)

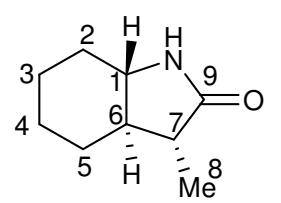

Following

general

procedure

$\mathbf{F}$,

(R)-ethyl

2-((1R,2S)-2-

nitrocyclohexyl)propanoate $((+)-5)(67.6 \mathrm{mg}, 0.29 \mathrm{mmol})$, gave the crude product which was purified by column chromatography (silica gel, ethyl acetate/hexane,

2/3) afforded the title compound as a white crystalline solid (5.2 $\mathrm{mg}, 0.03 \mathrm{mmol}, 12 \%) .[\alpha]_{D}^{23}$ $+17.7\left(c=0.1, \mathrm{CHCl}_{3}\right) ; \mathrm{MP}-91.2-91.9{ }^{\circ} \mathrm{C} ;{ }^{1} \mathrm{H} \mathrm{NMR}\left(400 \mathrm{MHz}, \mathrm{CDCl}_{3}\right)$ 1.15-1.17 $(3 \mathrm{H}, \mathrm{d}, J=7.5$ $\mathrm{Hz}, \mathrm{H}-8), 1.22-1.38$ (6 H, m, Cyc-H), 1.83-1.89 (3 H, m, Сyc-H), 1.98-2.08 (2 H, m, H-7 \& Cyc$\mathrm{H})$, 2.96-3.01 (1 H, dt, J=5.0 \& $12.5 \mathrm{~Hz}), 5.79(1 \mathrm{H}, \mathrm{br} \mathrm{s}, \mathrm{N}-H) ;{ }^{13} \mathrm{C} \mathrm{NMR}\left(100 \mathrm{MHz}, \mathrm{CDCl}_{3}\right)$ 12.5, 24.5, 26.0, 27.4, 30.8, 42.6, 52.6, 58.6, 180.7; IR $\left(\mathrm{cm}^{-1}\right) 3243(\mathrm{~m}), 2926(\mathrm{~s})<2862(\mathrm{~m})$, $2364(\mathrm{~m}), 1707$ (s), 1655 (s), 1381 (m), 1260 (s), 1075 (s), 867 (w), 800 (s); HRMS required for $\left(\mathrm{C}_{9} \mathrm{H}_{16} \mathrm{NO}^{+}\right)$154.1226; found 154.1224.

Determination of absolute configuration was by nitro reduction and cyclisation to the lactam and

${ }^{1} \mathrm{H}$ NMR analysis compared to literature values. Kojima, M.; Tomioka, Y.; Chem. Pharm. Bull.;

$1976,24,1613$. Conformation of relative configuration of this lactam was determined by $x$-ray crystallography (see page S87).

\section{(3aR,7aS)-3-benzyl-hexahydro-1H-indol-2(3H)-one, racemic (( \pm$)-36)$}

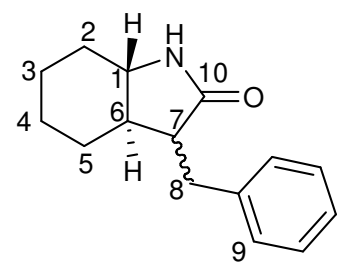

Following general procedure $\mathbf{F}$, racemic ethyl 2-(2-nitrocyclohexyl)-3phenylpropanoate $(( \pm)-6)(77.7 \mathrm{mg}, 0.25 \mathrm{mmol})$, gave the crude product which was purified by column chromatography (silica gel, ethyl acetate/hexane, 2/3) afforded the title compound as a white crystalline solid (20.6 mg, 0.09 mmol, 36 \%). MP - 102.8-103.2 ${ }^{\circ} \mathrm{C} ;{ }^{1} \mathrm{H}$ NMR $\left(400 \mathrm{MHz}, \mathrm{CDCl}_{3}\right)$ 0.93-1.40 (6 H, m, Cyc-H), 1.552.01 (3 H, m, Сус-H), 2.26-2.32 (1 H, m, Сус-H), 2.64-2.91 (2 H, m, Сус-H), 3.14-3.18 (1 H, dd, $J=4 \& 7.5 \mathrm{~Hz}, \mathrm{H}-1), 5.92(1 \mathrm{H}, \mathrm{br}, \mathrm{s}, \mathrm{N}-H), 7.10-7.26(5 \mathrm{H}, \mathrm{m}, \mathrm{H}-9) ;{ }^{13} \mathrm{C} \mathrm{NMR}\left(100 \mathrm{MHz}, \mathrm{CDCl}_{3}\right)$ $24.3,24.3,25.7,25.9,26.1,27.8,30.7,31.9,34.7,48.5,49.0,50.2,57.7,58.5,126.1,128.3$, 
128.4, 128.9, 129.2, 139.7, 179.4; IR $\left(\mathrm{cm}^{-1}\right) 3273(\mathrm{~m}), 2929$ (s), 2857 (m), 2375 (w), 1699 (s), $1437(w), 1381(w), 1275(w), 1099(w), 749(w), 700(w)$; HRMS required for $\left(C_{15} \mathrm{H}_{20} \mathrm{NO}^{+}\right)$ 230.1539; found 230.1539 .

\section{$(3 R, 3 \mathrm{a} R, 7 \mathrm{aS})-3-$ benzyl-hexahydro-1H-indol-2(3H)-one, chiral ((-)-36)}

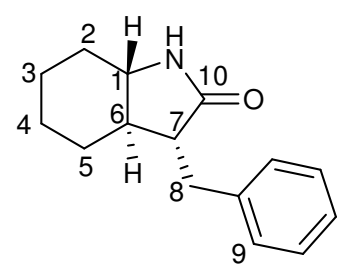

Following general procedure $\mathbf{F},(R)$-Ethyl 2-((1R,2S)-2-nitrocyclohexyl)-3phenylpropanoate $((+)-6)(50.0 \mathrm{mg}, 0.16 \mathrm{mmol})$, gave the crude product which was purified by column chromatography (silica gel, ethyl acetate/hexane, 2/3) afforded the title compound as a white crystalline solid (10.7 mg, 0.05 mmol, $29 \%) .[\alpha]_{D}^{23}-5.7\left(c=0.4, \mathrm{CHCl}_{3}\right)$; $\mathrm{MP}-102.5-103.1{ }^{\circ} \mathrm{C} ;{ }^{1} \mathrm{H} \mathrm{NMR}\left(400 \mathrm{MHz}, \mathrm{CDCl}_{3}\right)$ 0.981.09 (1 H, m, Сус-H), 1.13-1.36 (3 H, m, Сус-H), 1.35-1.47 (2 H, m, Сус-H), 1.63-1.66 (1 H, m, Сус-H), 1.74-1.77 (1 H, m, Сус-H), 1.94-1.98 (1 H, m, Сус-H), 2.33-2.39 (1 H, m, H-7), 2.72$2.77(1 \mathrm{H}, \mathrm{dd}, J=7.5 \& 12.5 \mathrm{~Hz}, \mathrm{H}-8), 2.92-2.98(1 \mathrm{H}, \mathrm{dt}, J=5.0 \& 12.5 \mathrm{~Hz}, \mathrm{H}-1), 3.21-3.25(1$ $\mathrm{H}, \mathrm{dd}, J=5.0$ \& $12.5 \mathrm{~Hz}, \mathrm{H}-8) ;{ }^{13} \mathrm{C} \mathrm{NMR}\left(100 \mathrm{MHz}, \mathrm{CDCl}_{3}\right)$ 24.3, 25.7, 27.9, 30.7, 34.7, 49.0, 50.2, 58.5, 126.1, 128.4, 129.2, 139.7, 179.3; IR $\left(\mathrm{cm}^{-1}\right) 3273(\mathrm{~m}), 2929(\mathrm{~s}), 2857(\mathrm{~m}), 2375(\mathrm{w})$, 1699 (s), 1437 (w), 1381 (w), 1275 (w), 1099 (w), 749 (w), 700 (w); HRMS required for $\left(\mathrm{C}_{15} \mathrm{H}_{20} \mathrm{NO}^{+}\right)$230.1539; found 230.1540 .

\section{Ethyl 2-((1R,2S)-2-aminocyclohexyl)acetate (37)}

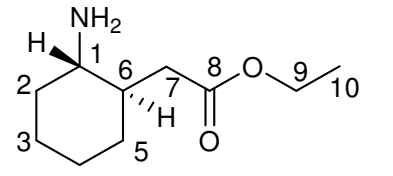

To a solution of ethyl 2-(2-nitrocyclohexyl)acetate $(100.0 \mathrm{mg}, 0.46 \mathrm{mmol})$ in ethanol $(8 \mathrm{~mL}$ ) was added Raney Nickel (one spatula), the solution was hydrogenated until TLC showed the reaction was complete. The reaction mixture was filtered through a pad of Celite, concentrated under reduced pressure and purified by column chromatography (silica gel, methanol/dichloromethane, 1/19) the title compound as a yellow oil (43.7 mg, 0.25 mmol, 54 \%). ${ }^{1} \mathrm{H}$ NMR (400 MHz, $\left.\mathrm{CDCl}_{3}\right)$ 1.01-1.08 (1 H, m, Cyc-H), 1.16-1.34 (1 
H, m, Сус-H), 1.24-1.27 (3 H, t, J = 7.5 Hz, H-10), 1.41-1.46 (1 H, m Сус-H), 1.55-1.79 (3 H, m, Сус-H), 1.87-1.90 (1 H, m, Сус-H), 2.06-2.45 (4 H, m, H-7 \& Сус-H), 2.66-2.71 (1 H, dd, J= 2.5 \& $10 \mathrm{~Hz}, \mathrm{H}-1), 4.10-4.16(2 \mathrm{H}, \mathrm{q}, \mathrm{J}=7.5 \mathrm{~Hz}, \mathrm{H}-9) ;{ }^{13} \mathrm{C} \mathrm{NMR}\left(100 \mathrm{MHz}, \mathrm{CDCl}_{3}\right)$ 14.2, 25.5, 25.7, 31.7, 36.8, 38.7, 42.5, 54.7, 60.2, 173.5; IR (cm $\left.{ }^{-1}\right) 3367(\mathrm{~m}), 2925(\mathrm{~s}), 2854(\mathrm{~m}), 1729(\mathrm{~s}), 1576$ (w), $1447(\mathrm{~m}), 1370(\mathrm{~m}), 1288(\mathrm{~m}), 1239(\mathrm{~m}), 1029(\mathrm{~m}), 933(\mathrm{w}), 859(\mathrm{w})$; HRMS required for $\left(\mathrm{C}_{10} \mathrm{H}_{20} \mathrm{NO}_{2}{ }^{+}\right)$186.1489; found 186.1485 .

\section{Ethyl 2-((1R,2S)-2-((S)-2-(benzyloxycarbonyl)-3-methylbutanamido)cyclohexyl)acetate ((-)-}

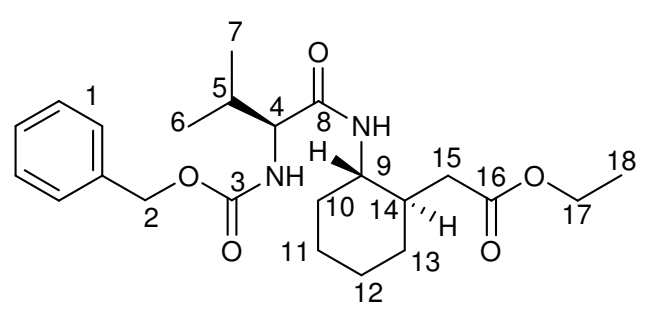

38)

To a solution of ethyl 2-((1R,2S)-2-aminocyclohexyl)acetate $37(43.7 \mathrm{mg}, 0.25 \mathrm{mmol})$ in dichloromethane $(5 \mathrm{~mL})$ was added hydroxybenzotriazole $(40.5 \mathrm{mg}, 0.30 \mathrm{mmol})$ and carbobenzyloxy-L-valine $(75.4 \mathrm{mg}, 0.30 \mathrm{mmol})$, this solution was cooled to $0{ }^{\circ} \mathrm{C}$ and stirred for 10 minutes before the addition of dicyclohexylcarbodiimide $(72.0 \mathrm{mg}, 0.35 \mathrm{mmol})$. This solution was left to warm to room temperature over night. The solution was filtered through a pad of celite and concentrated under reduced pressure to afford the crude product as a light yellow oil. Purification by column chromatography (silica gel, ethyl acetate/hexane, 3/7) the title compound as a crystalline solid $(80.6 \mathrm{mg}, 84 \%, 0.21 \mathrm{mmol}) .[\alpha]_{D}^{23}-15.4\left(c=0.5, \mathrm{CHCl}_{3}\right) ; \mathrm{MP}-147.8-148.2$ ${ }^{\circ} \mathrm{C} ;{ }^{1} \mathrm{H}$ NMR $\left(400 \mathrm{MHz}, \mathrm{CDCl}_{3}\right)$ 0.87-0.89 (3 H, d, J = 7.5 Hz, H-6 or 7), 0.93-0.95 (3 H, d, J=H6 or H-7), 1.10-1.36 (6 H, m, H-18 \& Cyc-H), 1.54-1.82 (4 H, m, Сyc-H), 1.93-1.96 (1 H, m, СycH), 2.04-2.14 (2 H, m, H-5 \& Сyc-H), 2.30-2.49 (1 H, m, H-4), 3.48-3.78 (2 H, m, H-15), 3.943.98 (1 H, m, H-17), 4.06-4.12 (2 H, m, H-9 \& H-17), 5.06-5.12 (2 H, m, H-2), 5.48-5.60 (1 H, m, $\mathrm{N}-H), 6.35-6.37(1 \mathrm{H}, \mathrm{m}, \mathrm{N}-H), 7.28-7.40(5 \mathrm{H}, \mathrm{m}, \mathrm{H}-1) ;{ }^{13} \mathrm{C} \mathrm{NMR}\left(100 \mathrm{MHz}, \mathrm{CDCl}_{3}\right)$ 14.2, 17.5, $17.7,19.2,19.4,25.1,25.4,30.7,30.9,32.1,33.6,38.6,39.2,48.1,53.2,60.4,60.5,60.6,67.0$, 128.0, 128.1, 128.5, 136.3, 156.5, 171.0, 172.9, 173.8; IR (cm $\left.{ }^{-1}\right) 3293(\mathrm{~m}), 2928(\mathrm{~m}), 1724(\mathrm{~m})$, 
1689 (s), 1643 (s), 1534 (s), 1452 (w), 1385 (w), 1340 (w), 1285 (m), 1245 (s), 1177 (w), 1116 (w), $1038(m), 697(w)$; HRMS required for $\left(\mathrm{C}_{23} \mathrm{H}_{35} \mathrm{~N}_{2} \mathrm{O}_{5}{ }^{+}\right)$419.2540; found 419.2536.

\section{(R)-2-((1R,2S)-2-nitrocyclohexyl)propan-1-ol (39)}

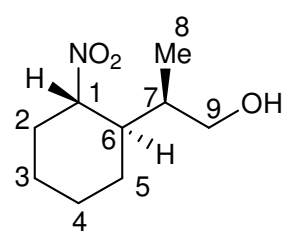

Following general procedure D, $\quad(R)$-ethyl 2-((1R,2S)-2nitrocyclohexyl)propanoate $((+)-5) \quad(50.0 \mathrm{mg}, 0.22 \mathrm{mmol})$ gave the crude product which was purified by column chromatography (silica gel, ethyl acetate/hexane, 3/7) to afford the title compound as a clear colourless oil $(31.4 \mathrm{mg}, 76 \%, 0.17$ mmol). ${ }^{1} \mathrm{H}$ NMR (400 MHz, $\left.\mathrm{CDCl}_{3}\right)$ 0.85-0.87 (3 H, d, J = $\left.7.5 \mathrm{~Hz}, \mathrm{H}-8\right), 1.05-1.15(1 \mathrm{H}, \mathrm{m}, \mathrm{Cyc}-$ H), 1.22-1.39 (2 H, m, Сyc-H), 1.58-1.64 (2 H, m, H-7 \& Cyc-H), 1.73-1.80 (2 H, m, Cyc-H \& OH, 1.85-1.98 (2 H, m, Суc-H), 2.24-2.32 (2 H, m, H-6 \& Cyc-H), 3.45-3.54 (2 H, m, H-9), 4.43$4.50(1 \mathrm{H}, \mathrm{dt}, J=5.0 \& 12.5 \mathrm{~Hz}, \mathrm{H}-1) ;{ }^{13} \mathrm{C}$ NMR $\left(100 \mathrm{MHz}, \mathrm{CDCl}_{3}\right)$ 10.5, 14.8, 23.4, 24.5, 24.7, 25.2, 32.3, 32.8, 35.6, 36.1, 41.1, 64.8, 65.6, 88.7; IR $\left(\mathrm{cm}^{-1}\right) 3368(\mathrm{~m}), 2938(\mathrm{~s}), 2359(\mathrm{w}), 1547$ (s), $1450(m), 1376(m), 1248(w), 1038(m), 894(w), 734(m), 668(w)$.

\section{(R)-2-((1R,2S)-2-nitrocyclohexyl)propanoic acid (40)}

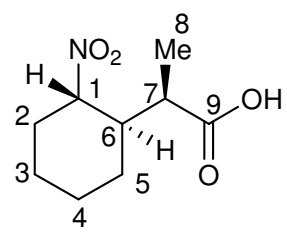

To a solution of $(R)-2-((1 R, 2 S)-2-$ nitrocyclohexyl)propan-1-ol 39 (30.0 mg, 0.16 $\mathrm{mmol})$ in acetone $(2 \mathrm{~mL})$ at $0{ }^{\circ} \mathrm{C}$ was added Jones reagent $((1 \mathrm{~mL}, 0.24 \mathrm{mmol})$ http://www.organic-chemistry.org/chemicals/oxidations/jones-reagent.shtm).

This solution was stirred overnight while warming to room temperature, excess isopropanol was added and the mixture was stirred for 10 minutes. The mixture was filtered, diluted with hydrochloric acid (2 M, $1 \mathrm{~mL})$ and extracted with diethyl ether $(5 \mathrm{~mL})$. The aqueous layer was washed with diethyl ether $(3 \times 5 \mathrm{~mL})$, the combined organic layers were washed with brine $(15$ $\mathrm{mL}$ ), dried over $\mathrm{MgSO}_{4}$, filtered and concentrated under reduced pressure to give the crude product. Purification by column chromatography (silica gel, ethyl acetate/hexane, 2/3) afforded the title compound as a clear colourless oil $(29.0 \mathrm{mg}, 90 \%, 0.14 \mathrm{mmol}) .{ }^{1} \mathrm{H} \mathrm{NMR}(400 \mathrm{MHz}$, 
$\left.\mathrm{CDCl}_{3}\right)$ 1.13-1.15 (3 H, d, J = 7.5 Hz, H-8), 1.24-1.40 (3 H, m, Сyc-H), 1.72-1.80 (2 H, m, CycH), 1.85-2.01 (2 H, m, Сус-H), 2.27-2.31 (1 H, m, Сус-H), 2.50-2.65 (2 H, m, H-7 \& Сус-H), 4.40-4.46 (1 H, dt, $J=5.0 \& 12.5 \mathrm{~Hz}, \mathrm{H}-1) ;{ }^{13} \mathrm{C} \mathrm{NMR}\left(100 \mathrm{MHz}, \mathrm{CDCl}_{3}\right)$ 9.5, 22.7, 24.3, 24.6, 25.0, 31.6, 32.0, 32.8, 39.3, 42.2, 87.7, 180.3; IR (cm $\left.{ }^{-1}\right) 2942$ (s), $2864(\mathrm{~m}), 1712$ (s), 1547 (s), $1453(m), 1375(m), 1299(w), 1242(m), 1142(m), 1113(w), 1053(w), 988(w), 900(w), 863$ (w), 735 (w); HRMS required for $\left(\mathrm{C}_{9} \mathrm{H}_{15} \mathrm{O}_{4} \mathrm{NNa}\right) 224.0899$; found 224.0894 .

\section{(S)-Methyl 3-methyl-2-((R)-2-((1R,2S)-2-nitrocyclohexyl)propanamido)butanoate ((+)-41)}

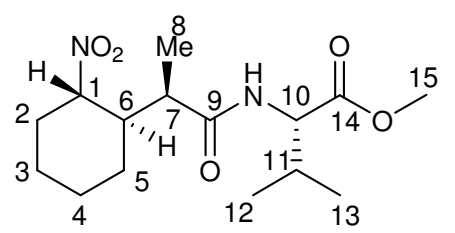

To a solution of $(R)-2-((1 R, 2 S)-2-$ nitrocyclohexyl)propanoic acid $\mathbf{4 0}$ (35.0 $\mathrm{mg}, 0.17 \mathrm{mmol})$ in dichloromethane $(6 \mathrm{~mL})$ was added, L-Valine methyl ester hydrochloride (32.1 mg, $0.19 \mathrm{mmol}), \quad N$-Ethyl- $N^{\prime}-(3-$ dimethylaminopropyl)carbodiimide hydrochloride $(40.1 \mathrm{mg}, 0.21 \mathrm{mmol})$, diisopropylethyl amine $(37 \mu \mathrm{L}, 0.21 \mathrm{mmol})$ and dimethyl amino pyridine $(2 \mathrm{mg}, 0.02 \mathrm{mmol})$. The mixture was stirred for 24 hours, was then acidified with aqueous sodium hydrogen sulphate extracted with ethyl acetate $(3 \times 10 \mathrm{~mL})$. The combined organic layers were washed with saturated sodium hydrogen carbonate $(20 \mathrm{~mL})$ and brine $(20 \mathrm{~mL})$, dried over $\mathrm{MgSO}_{4}$, filtered and concentrated under reduced pressure to give the crude product. Purification by column chromatography (silica gel, ethyl acetate/hexane, 1/4) gave the title compound as a clear colourless oil $(11.9 \mathrm{mg}, 22 \%, 0.04$ mmol). $[\alpha]_{D}^{23}+6.0\left(c=0.4, \mathrm{CHCl}_{3}\right) ;{ }^{1} \mathrm{H} \mathrm{NMR}\left(400 \mathrm{MHz}, \mathrm{CDCl}_{3}\right)$ 0.91-0.95 $(6 \mathrm{H}, \mathrm{dd}, J=7.5 \& 12.0$ $\mathrm{Hz}, \mathrm{H}-12$ \& H-13), 1.10-1.12 (3 H, d, J = 7.5 Hz, H-8), 1.22-1.33 (3 H, m, Cyc-H), 1.75-1.77 (1 H, m, Сус-H), 1.84-1.93 (3 H, m, H-6 \& Сyc-H), 2.14-2.21 (1 H, m, H-11), 2.28-2.42 (3 H, m, H-7 \& Cyc-H), $3.73(3 \mathrm{H}, \mathrm{s}, \mathrm{H}-15), 4.42-4.49(1 \mathrm{H}, \mathrm{dt}, J=5.0$ \& $12.5 \mathrm{~Hz}, \mathrm{H}-1), 4.53-4.56(1 \mathrm{H}, \mathrm{dd}, J=$ 5.0 \& $7.5 \mathrm{~Hz}, \mathrm{H}-10), 5.91-5.93(1 \mathrm{H}, \mathrm{m}, \mathrm{N}-H) ;{ }^{13} \mathrm{C} \mathrm{NMR}\left(100 \mathrm{MHz}, \mathrm{CDCl}_{3}\right)$ 10.2, 17.8, 19.0, 24.3, 24.6, 24.8, 31.2, 32.3, 40.6, 43.9, 40.6, 43.9, 52.2, 56.8, 88.1, 172.4, 173.7; IR (cm $\left.{ }^{-1}\right) 3323(w)$, $2939(m), 2869(m), 2360(m), 2340(w), 1742(m), 1657(m), 1546(s), 1450(m), 1371(m)$, 
1308 (w), $1264(w), 1203(m), 1154(m), 897(w), 734(w)$; HRMS required for $\left(\mathrm{C}_{15} \mathrm{H}_{27} \mathrm{O}_{5} \mathrm{~N}_{2}{ }^{+}\right)$ 315.1914 ; found 315.1913 . 


\section{NMR Data}
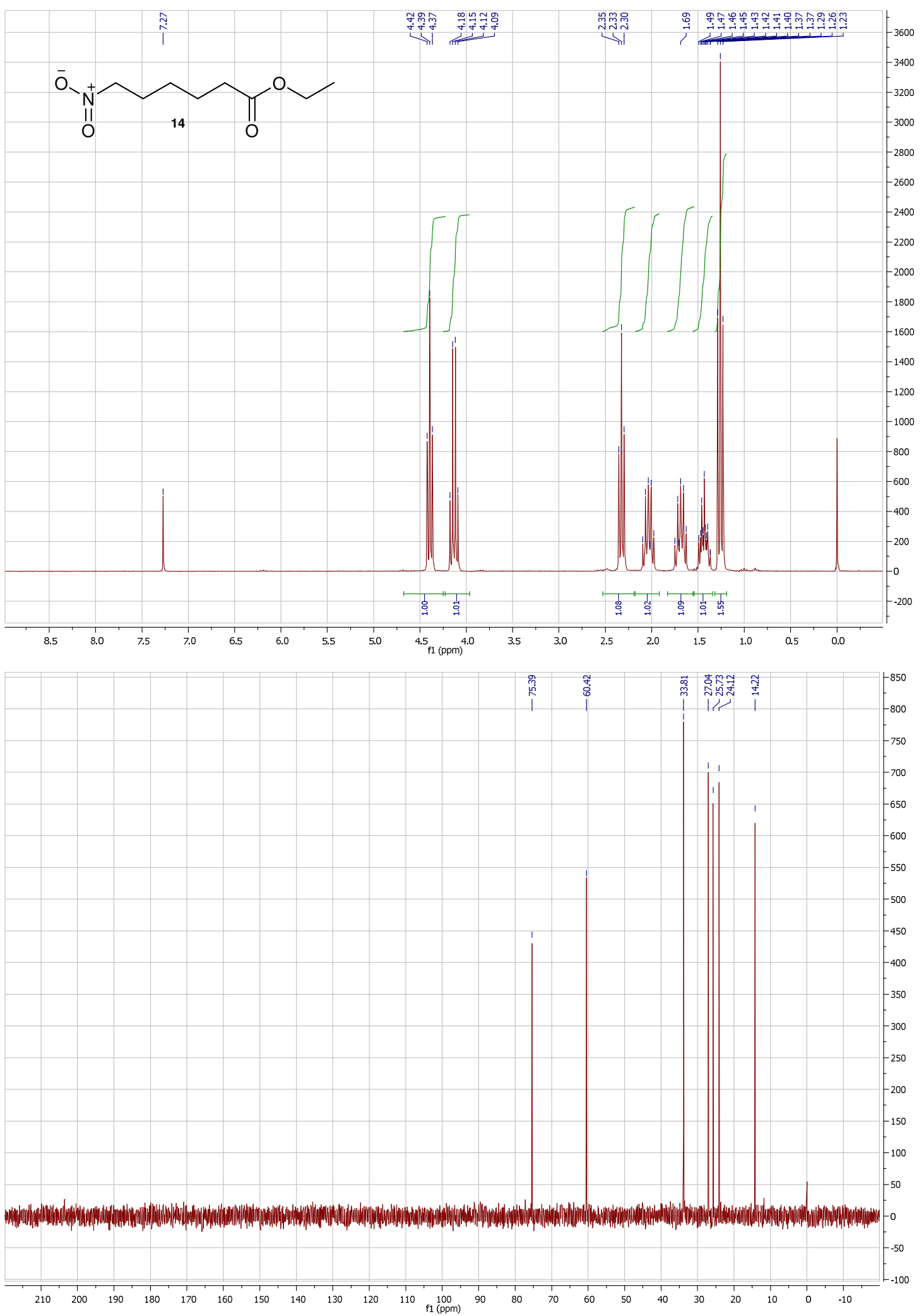


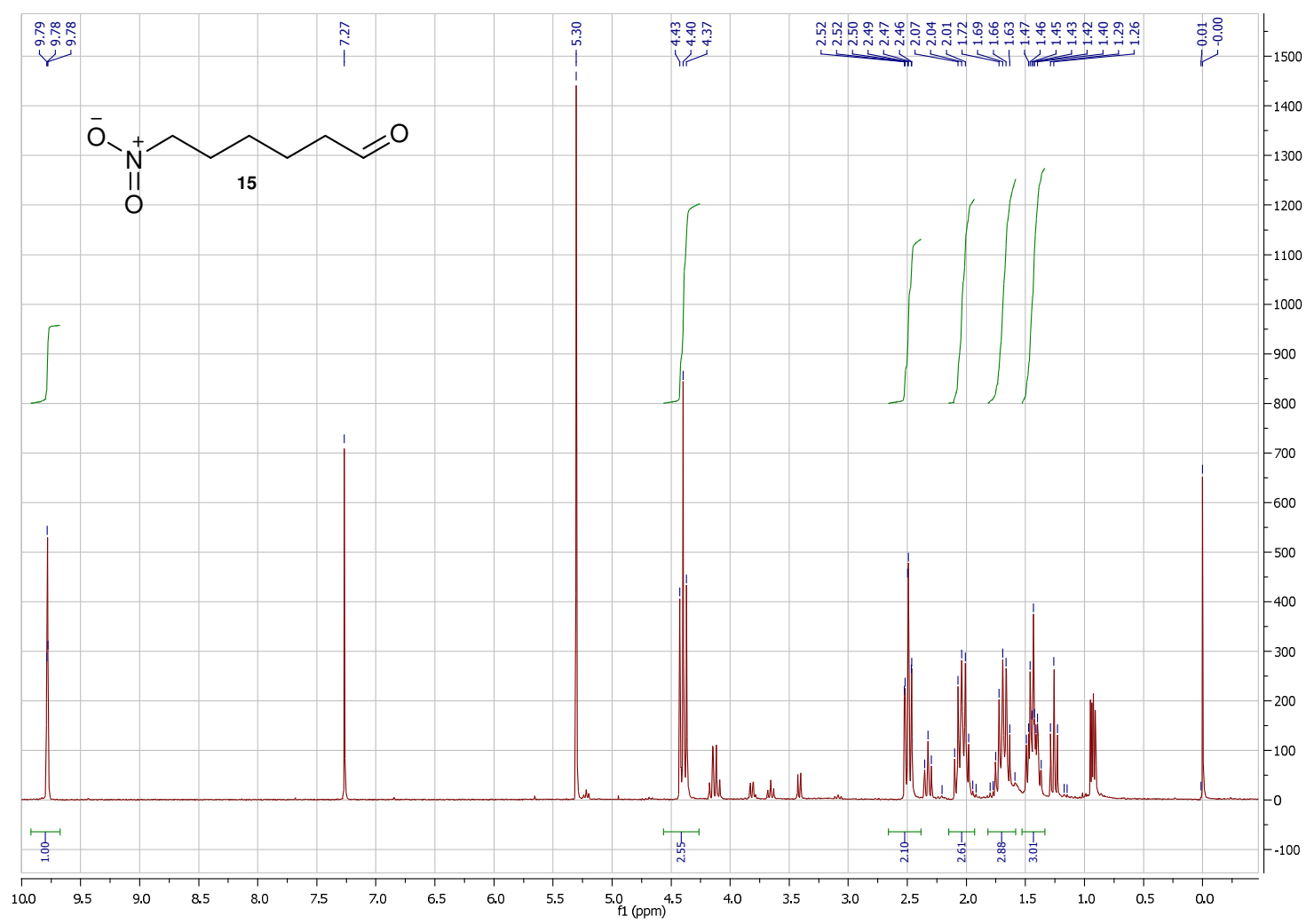



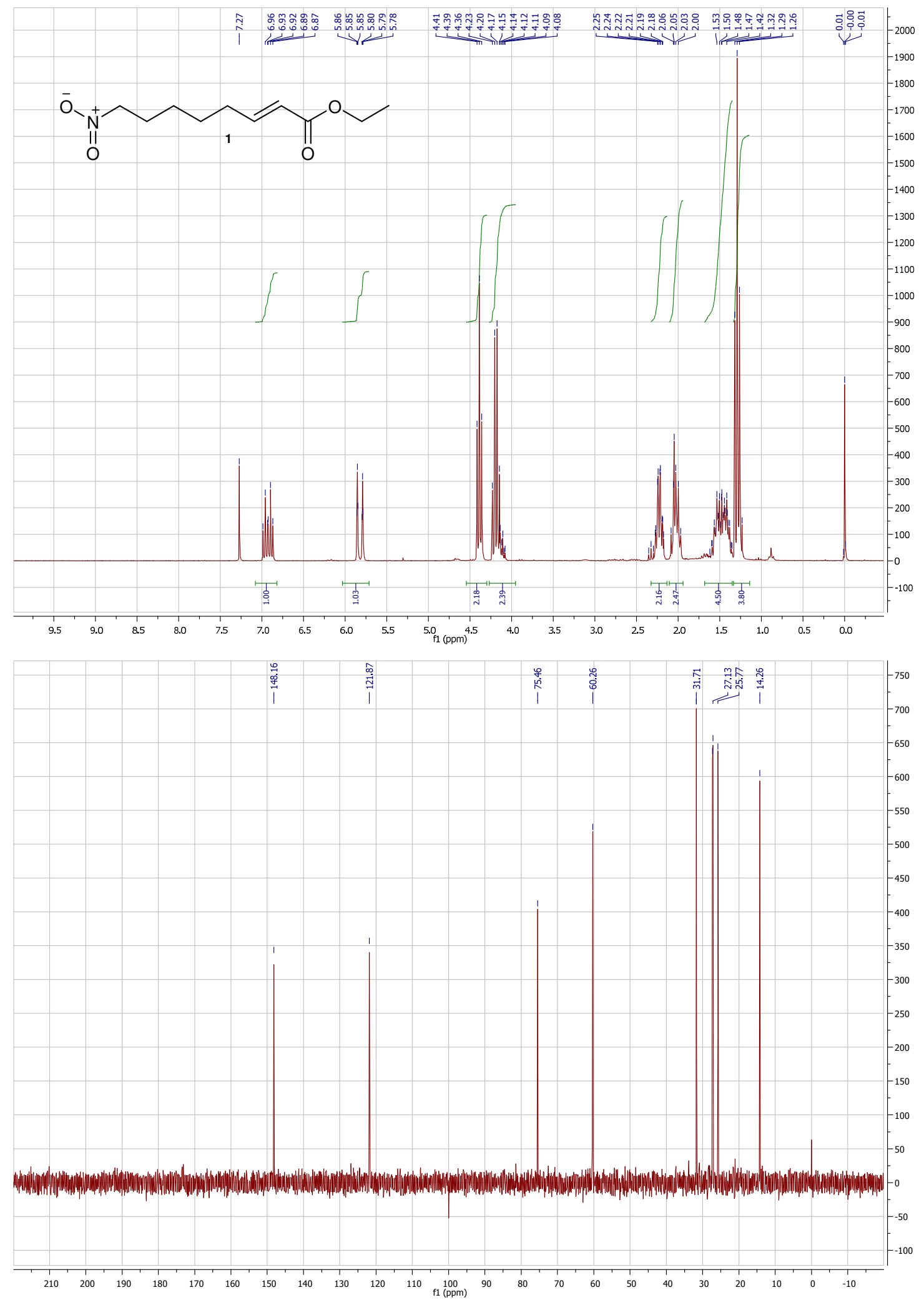


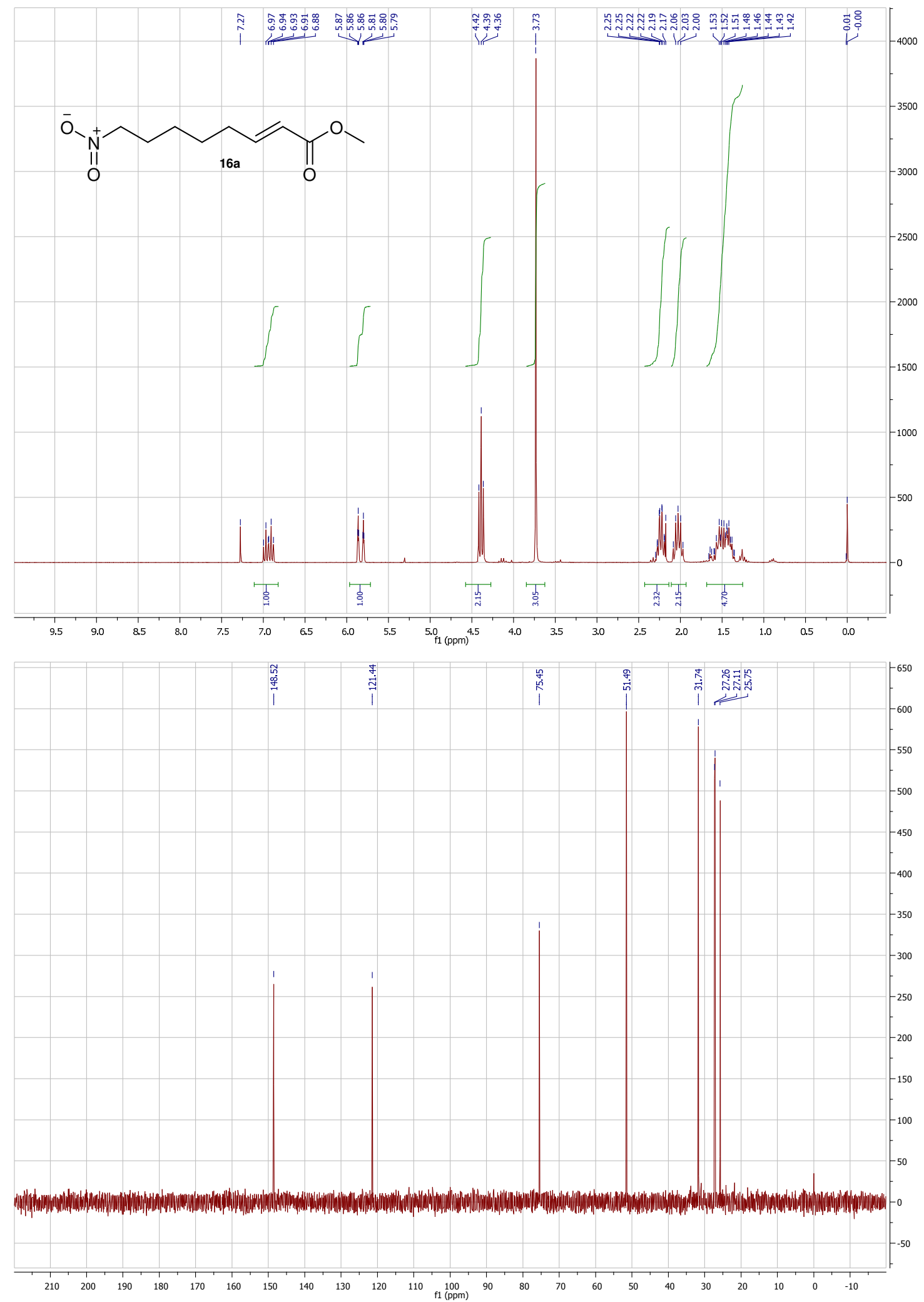



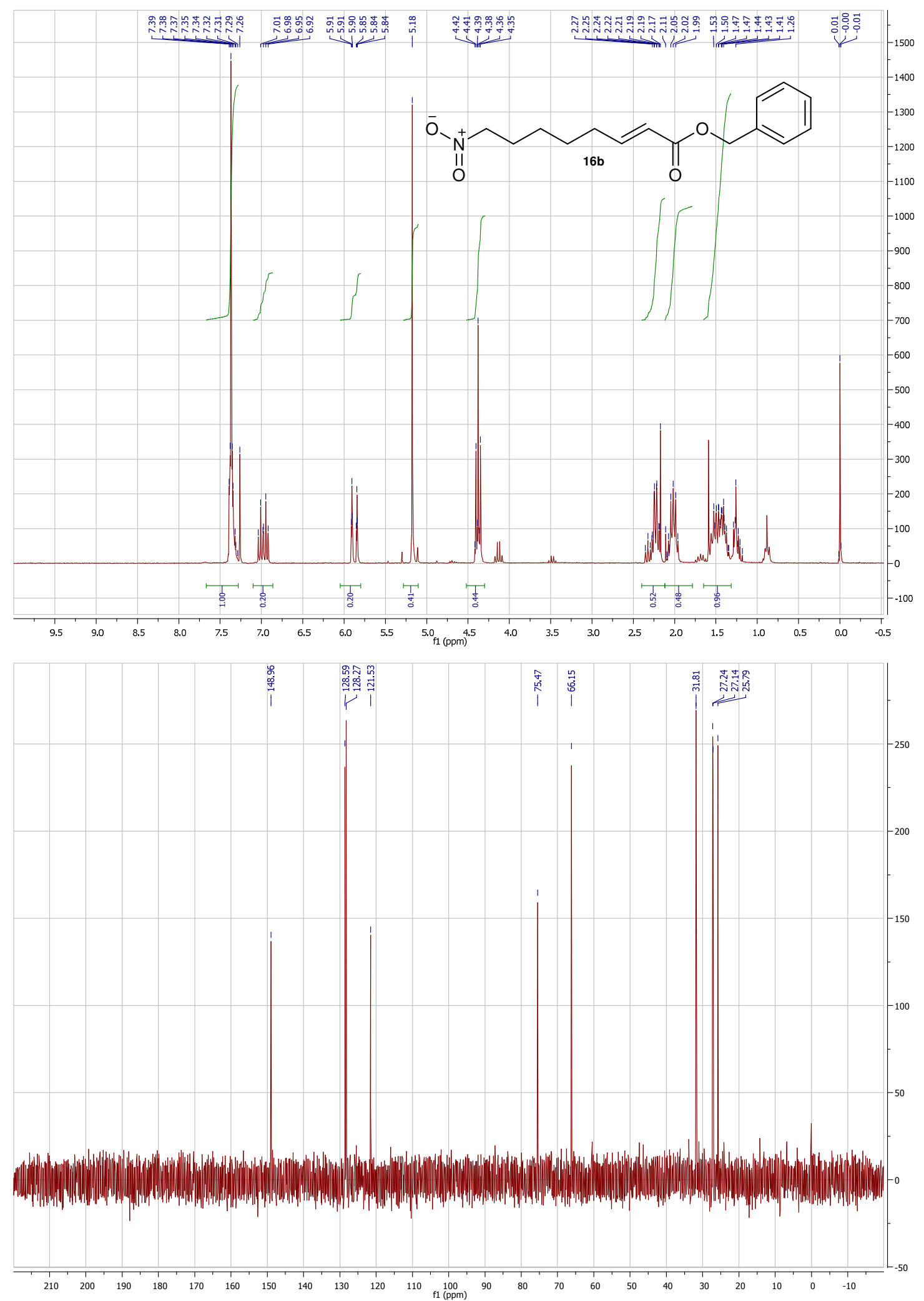

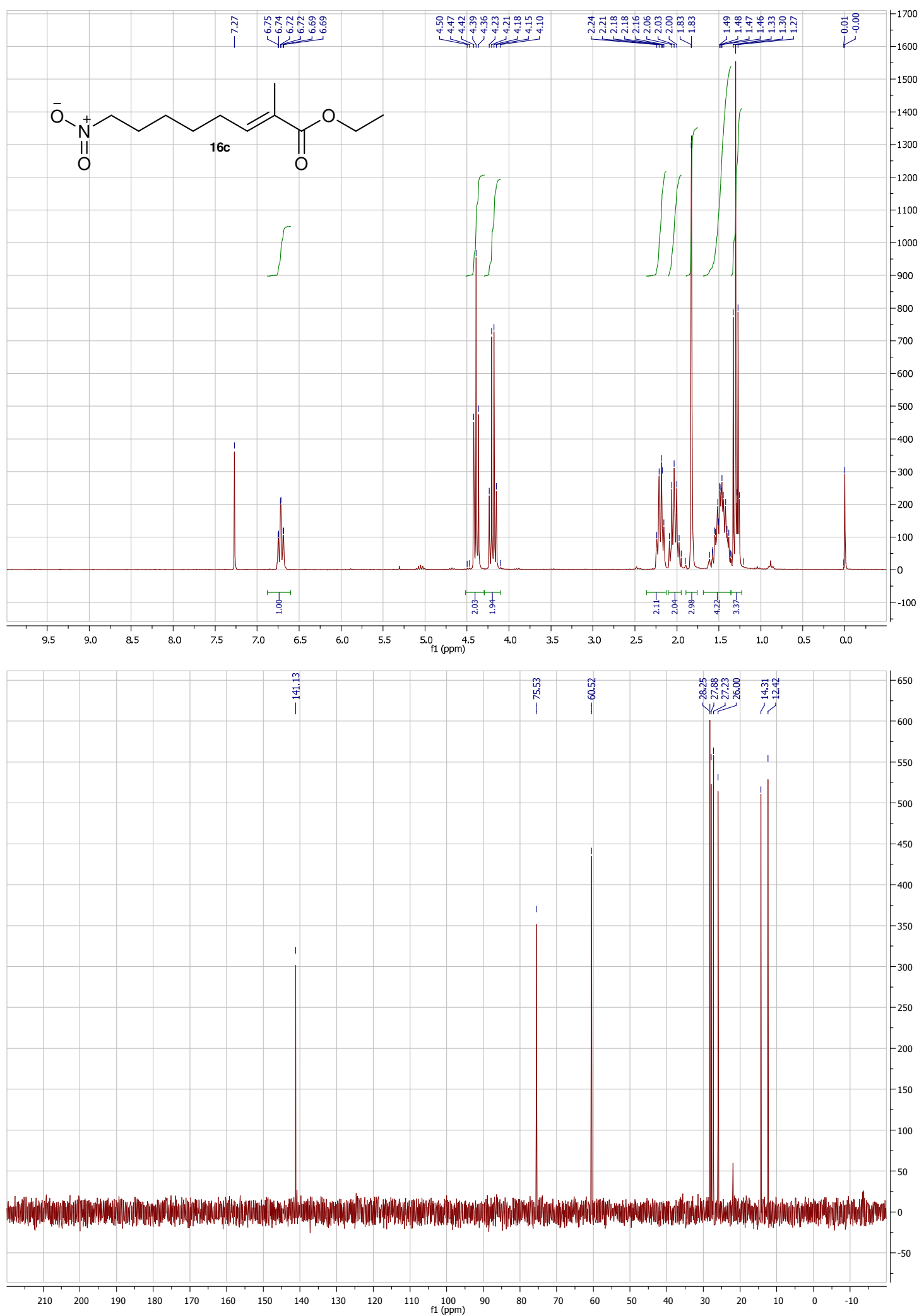


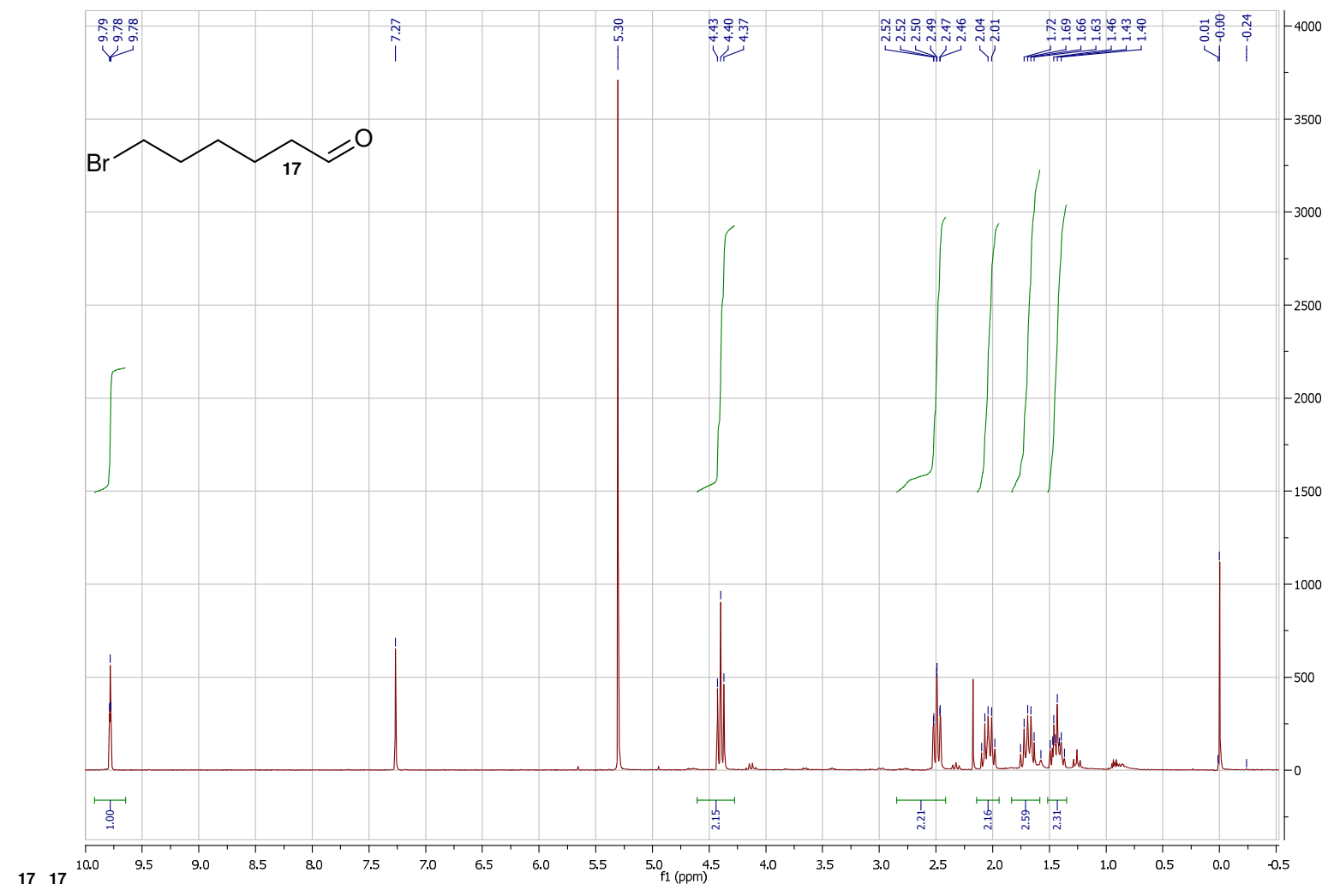



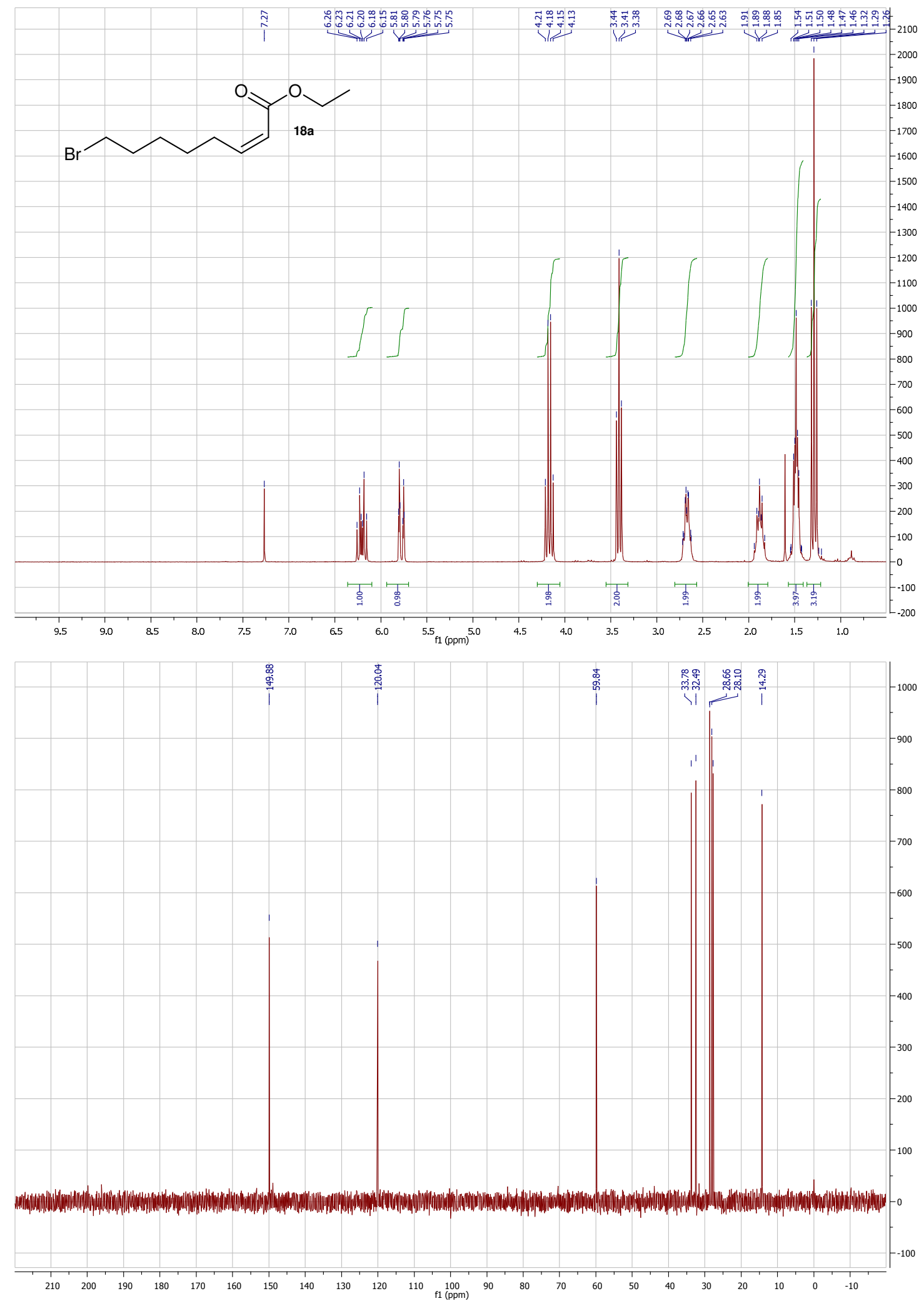

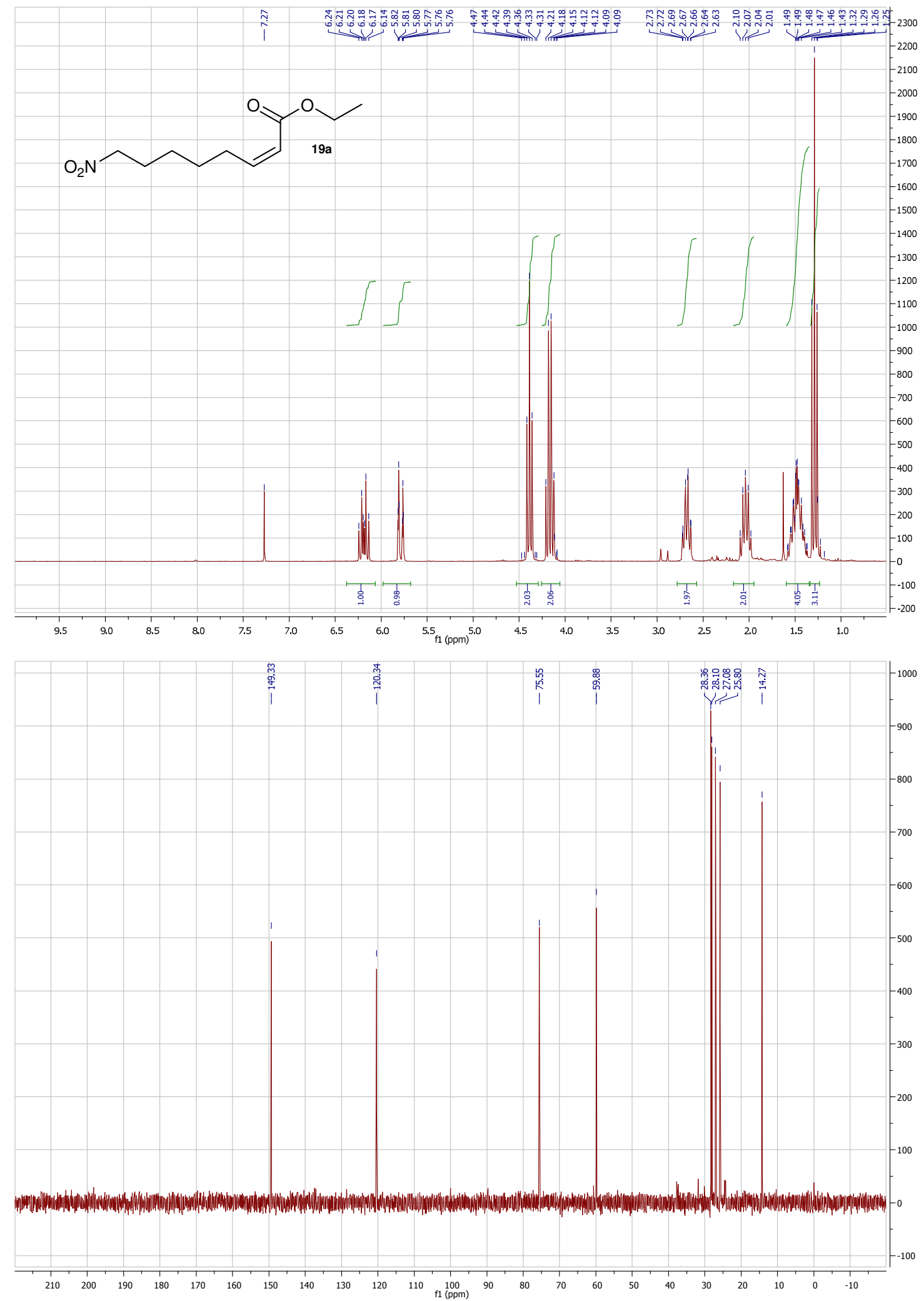

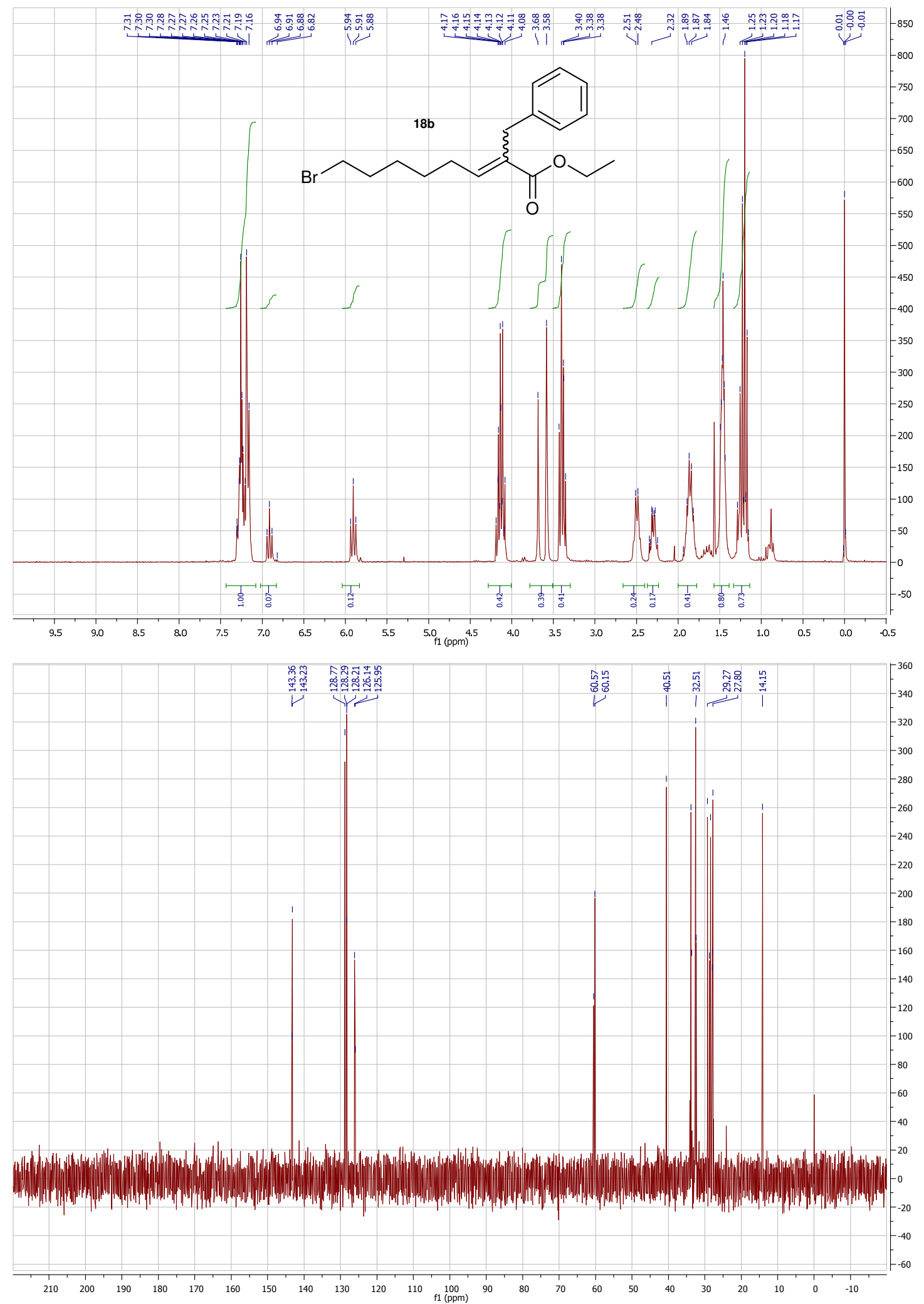

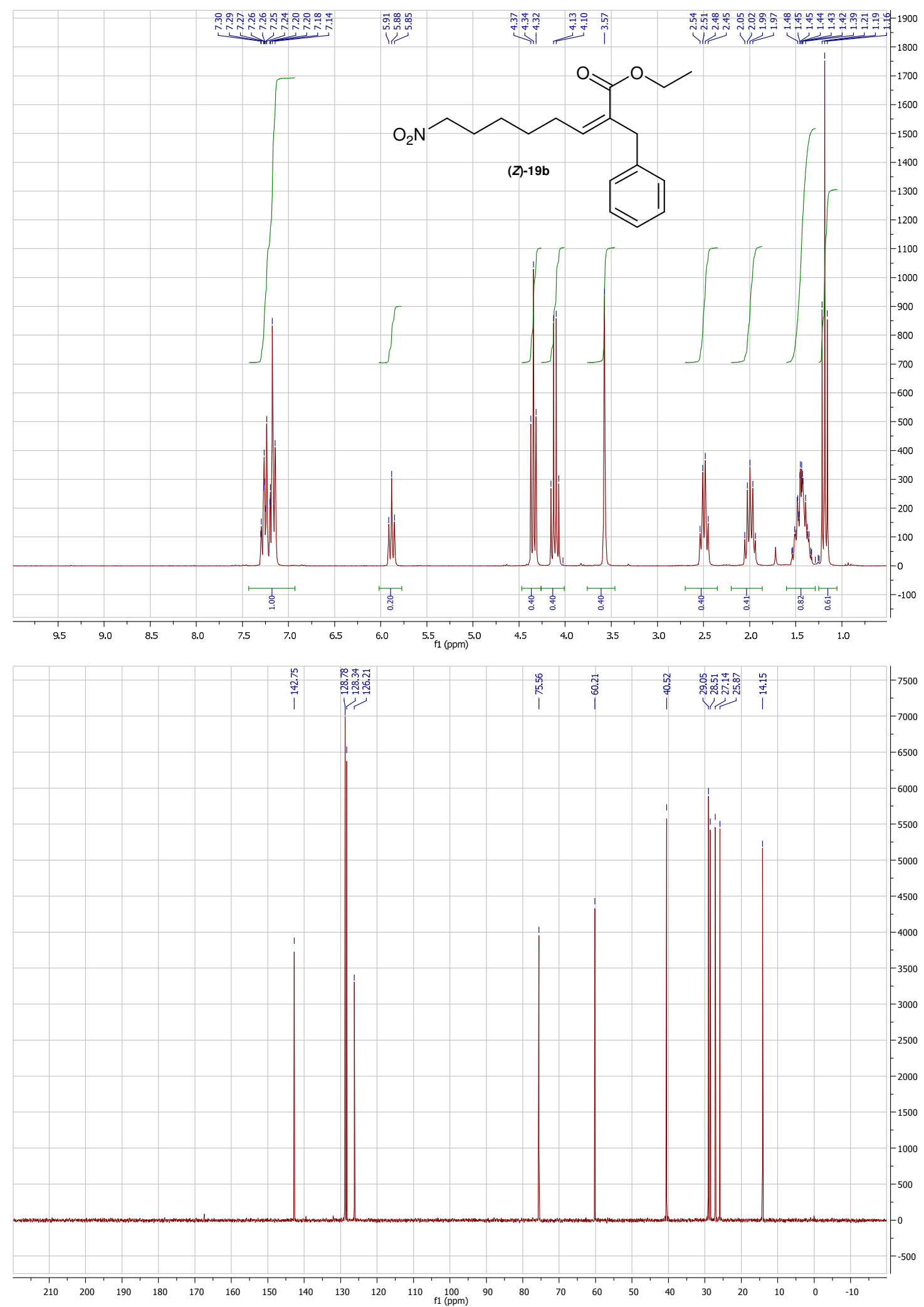

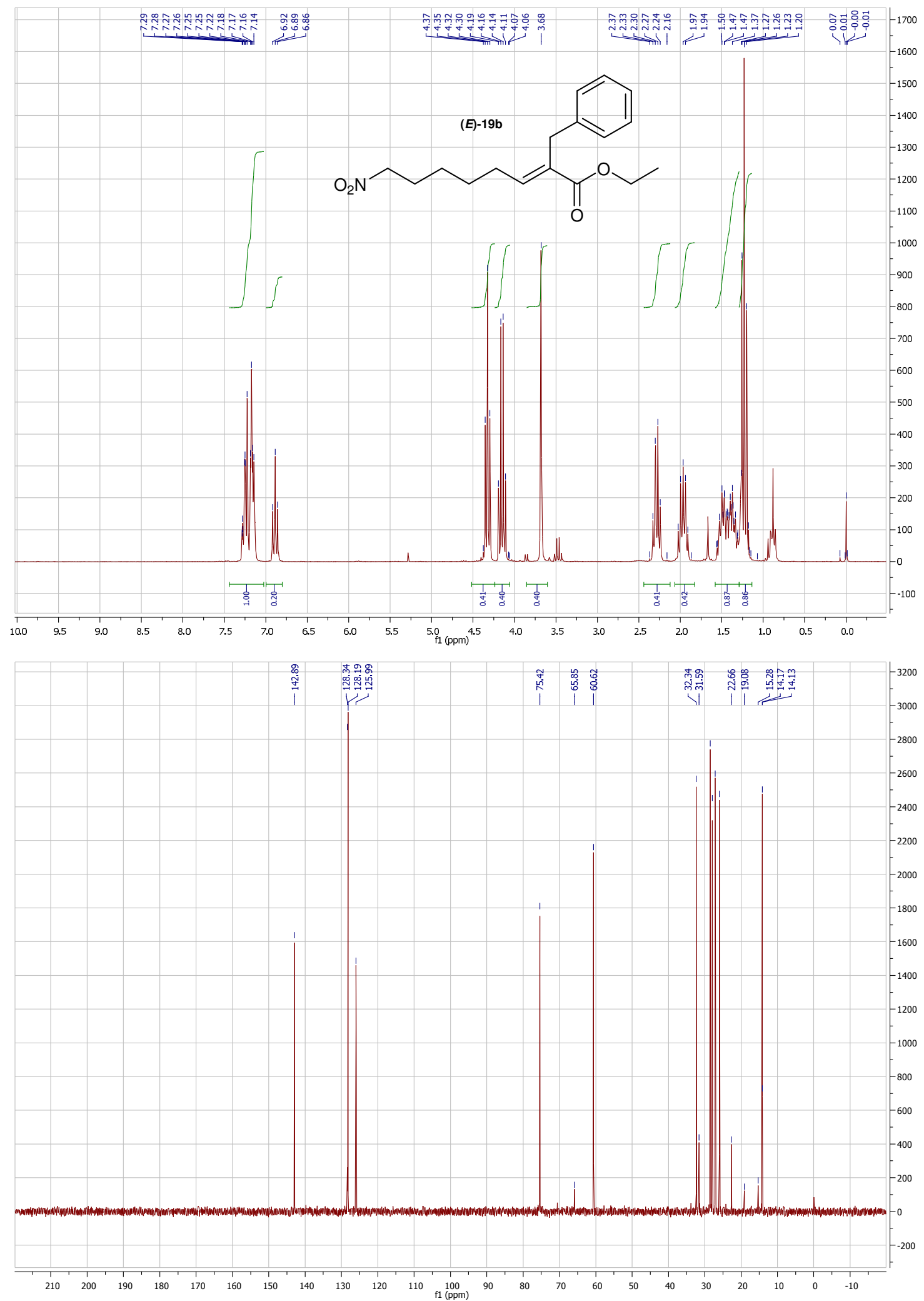

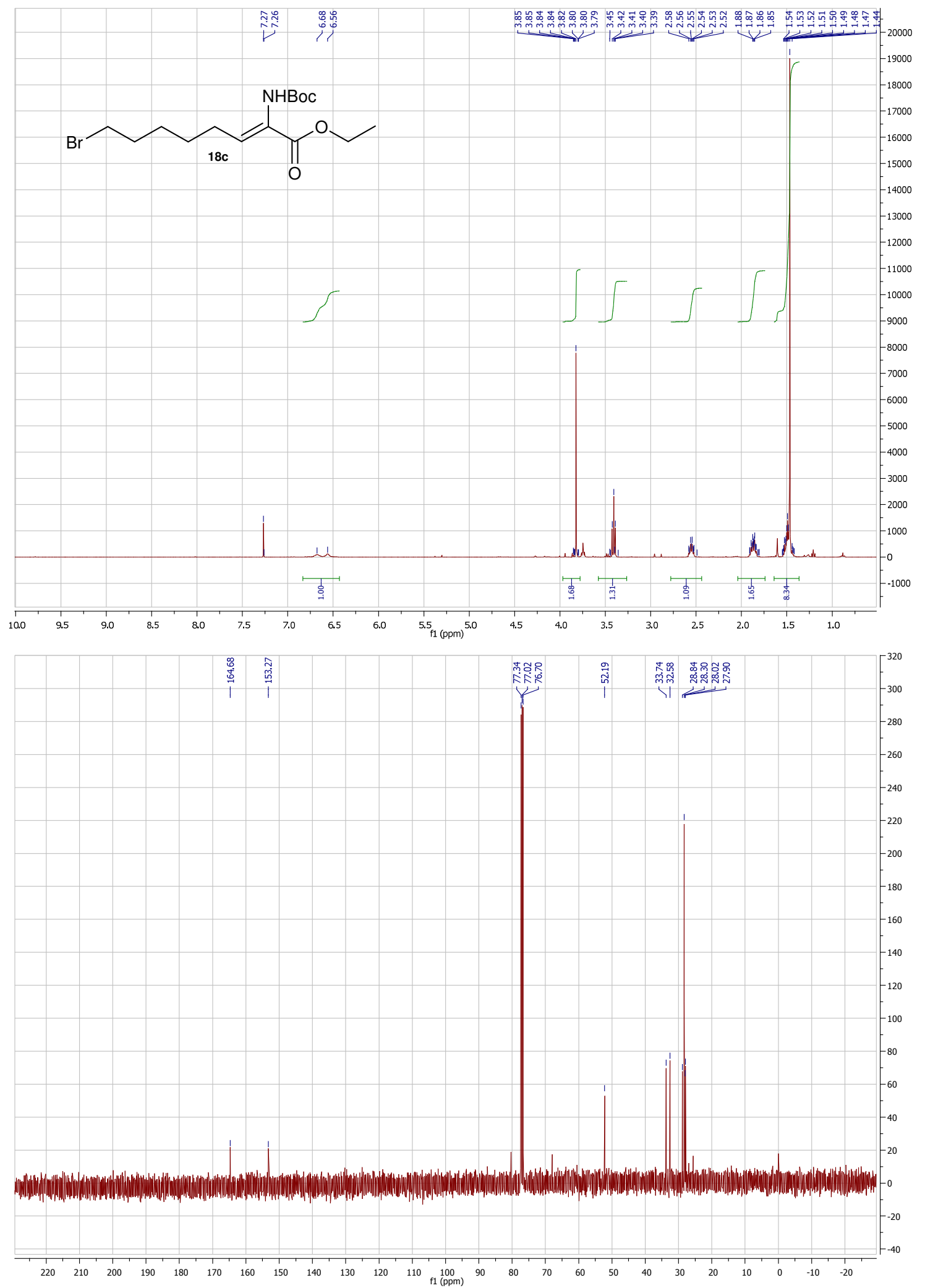

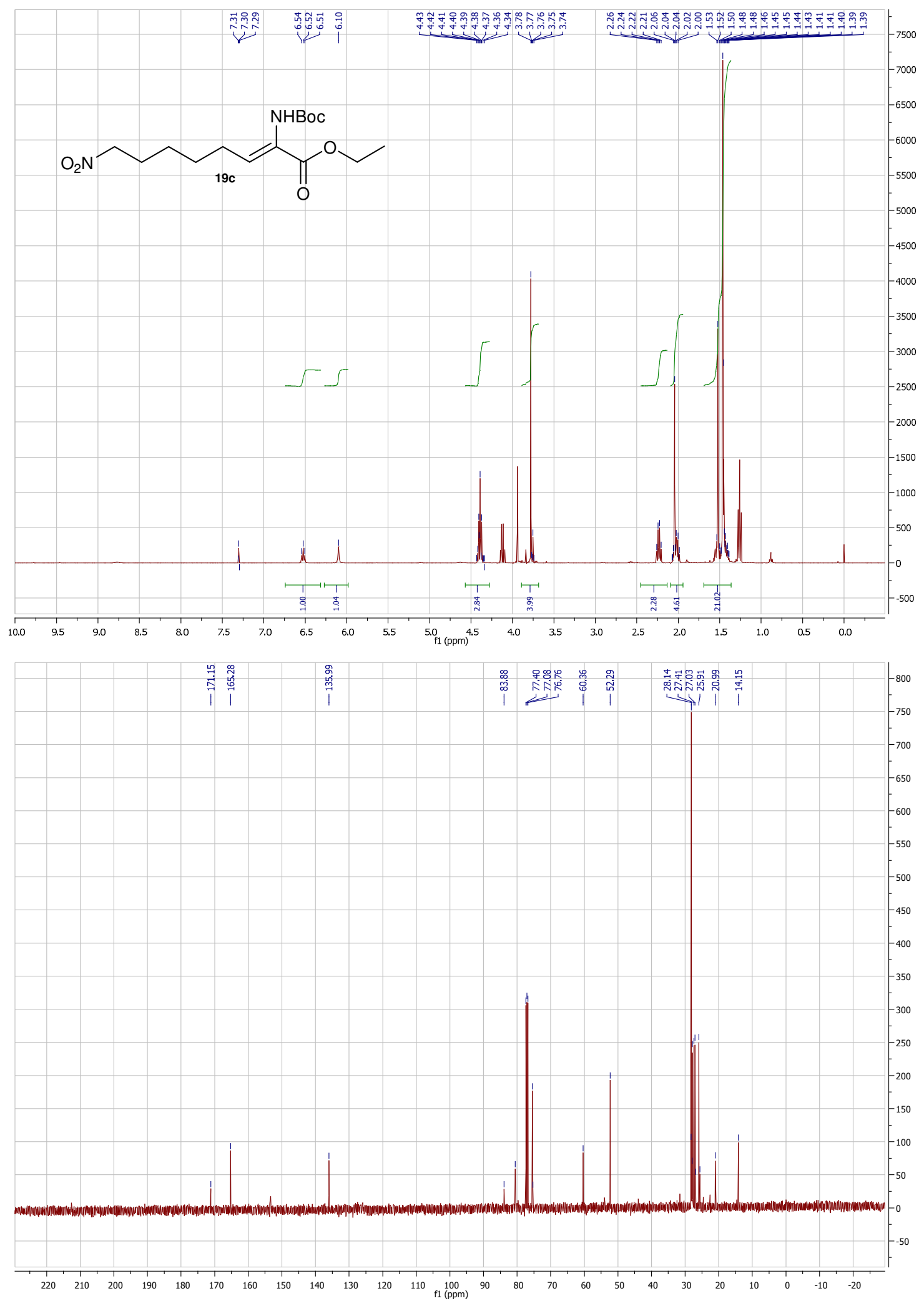

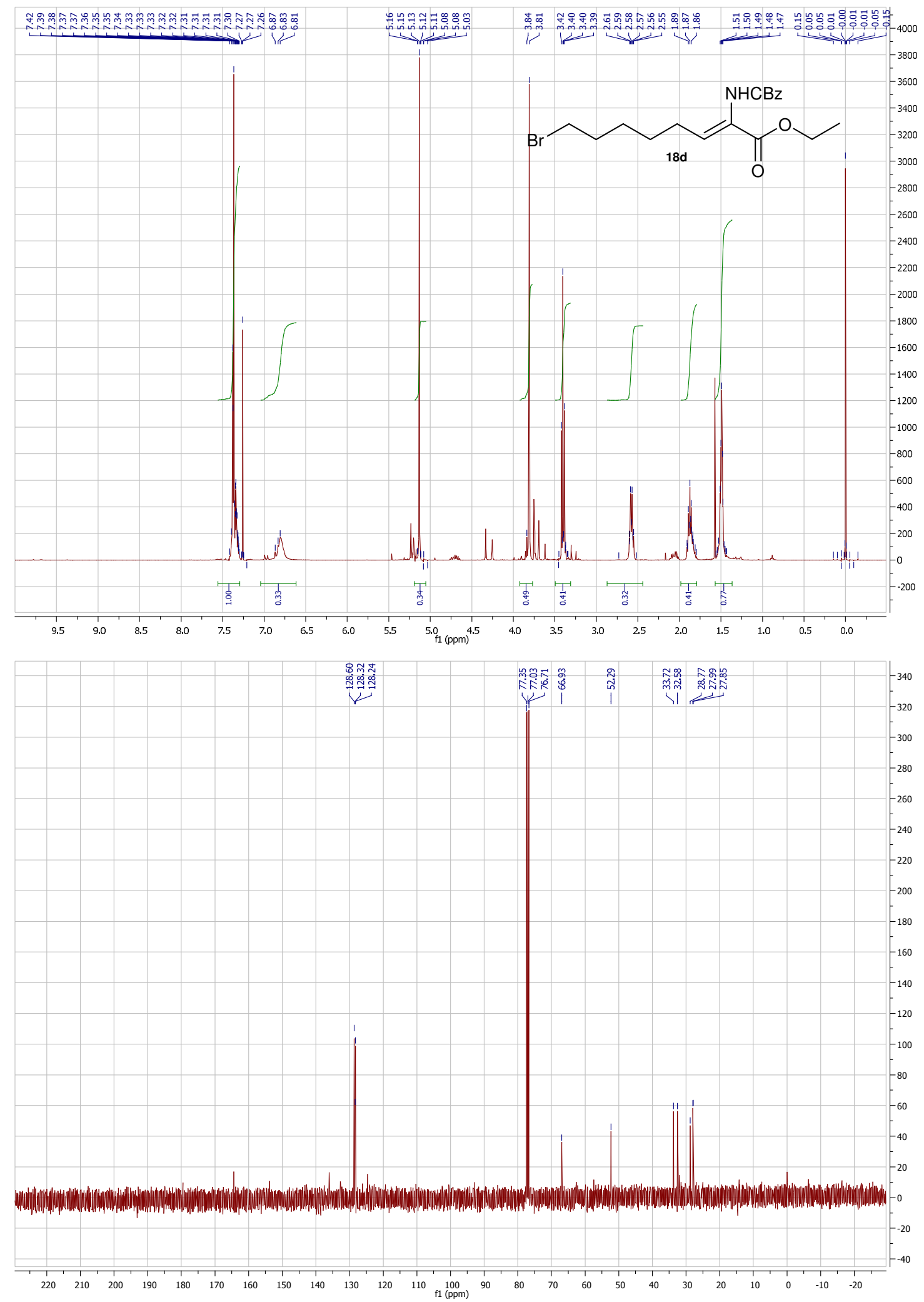

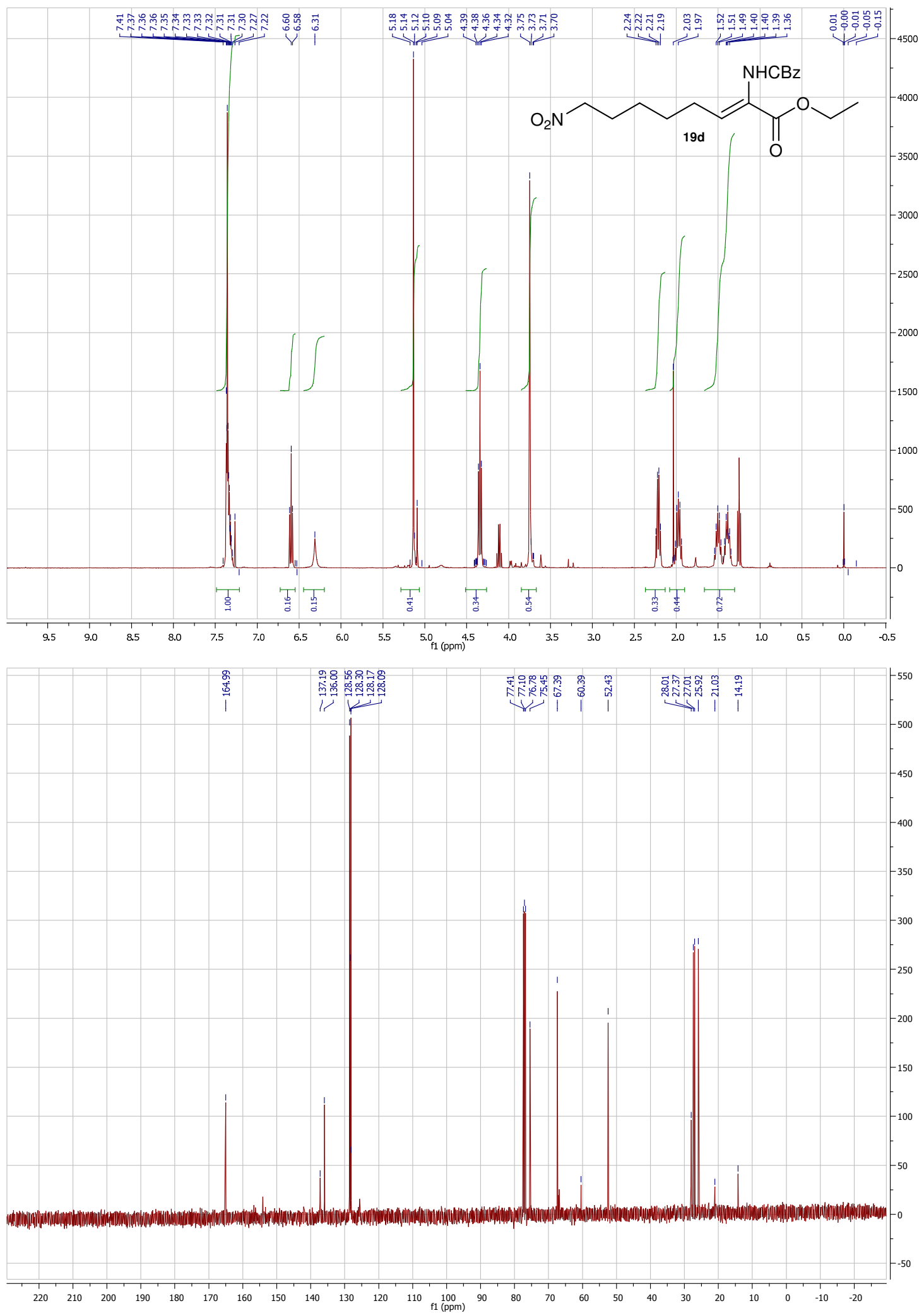

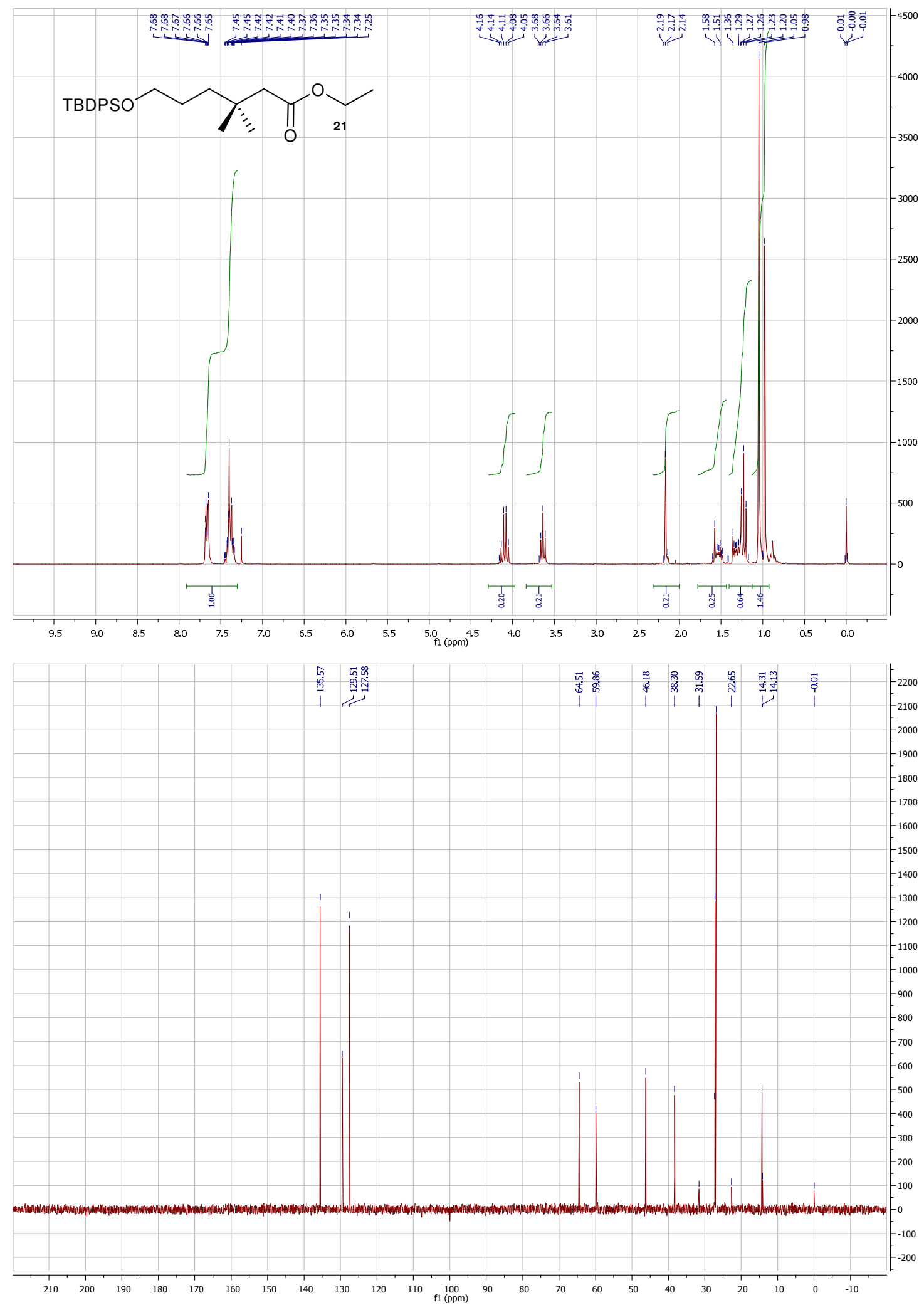

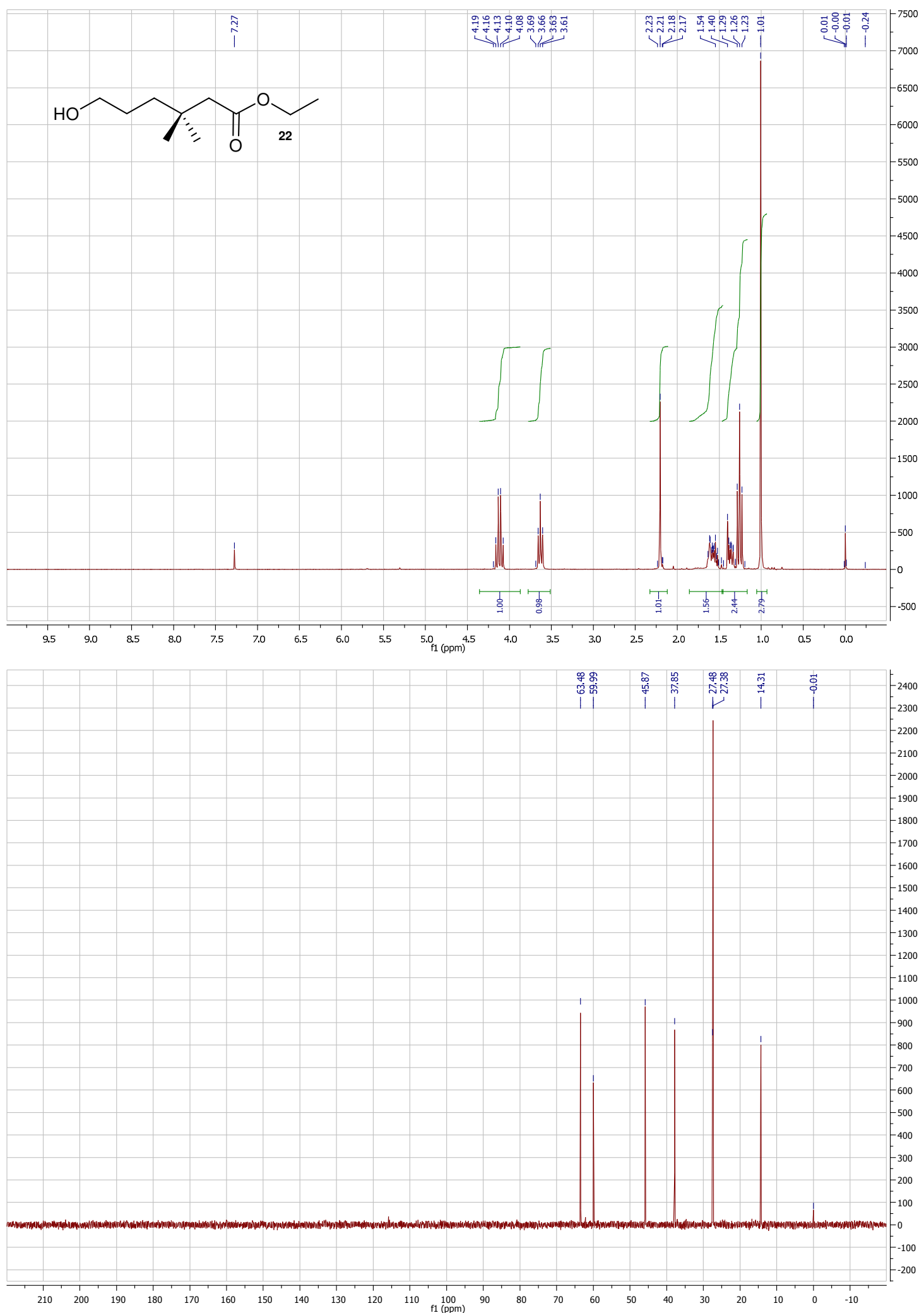


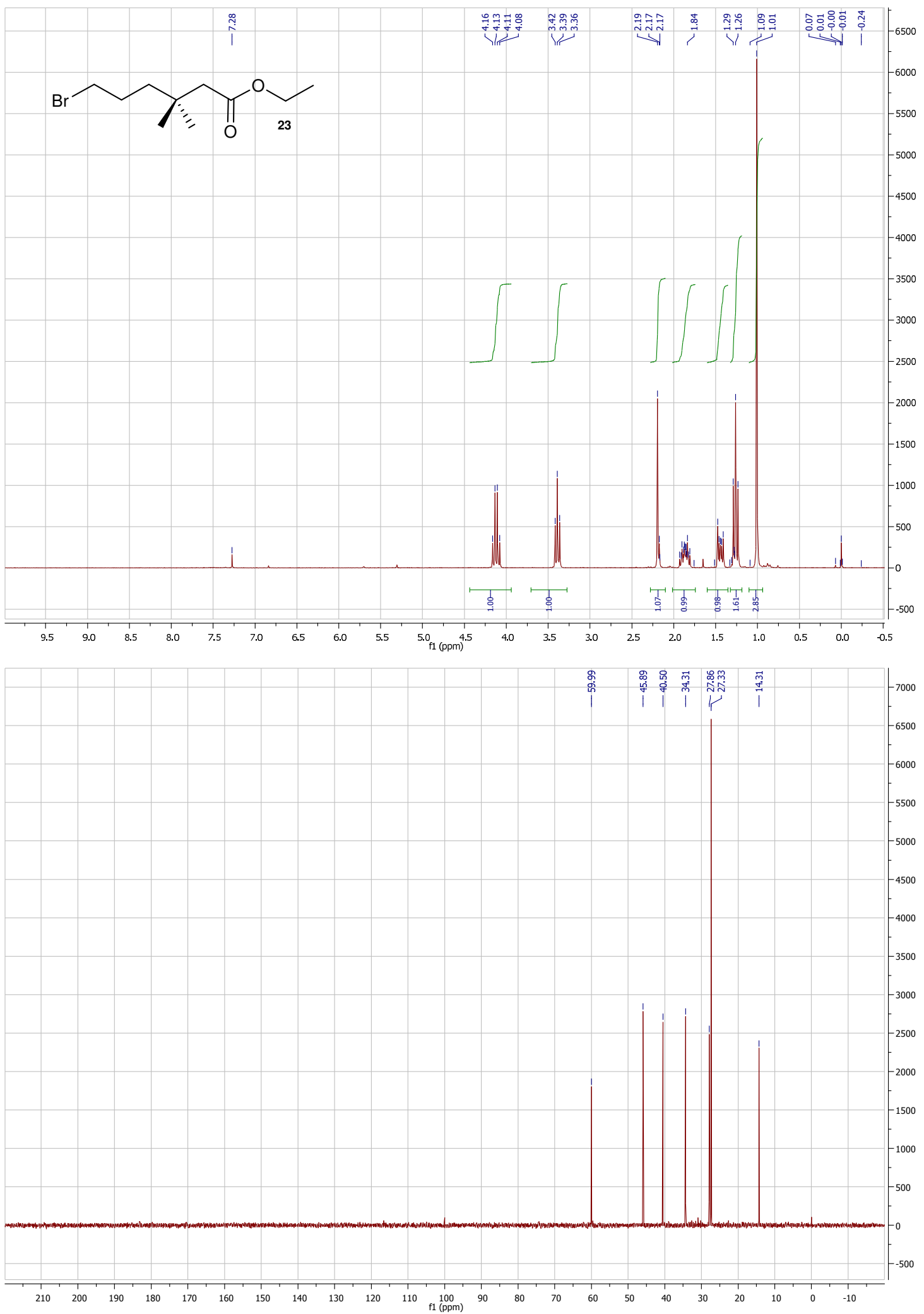



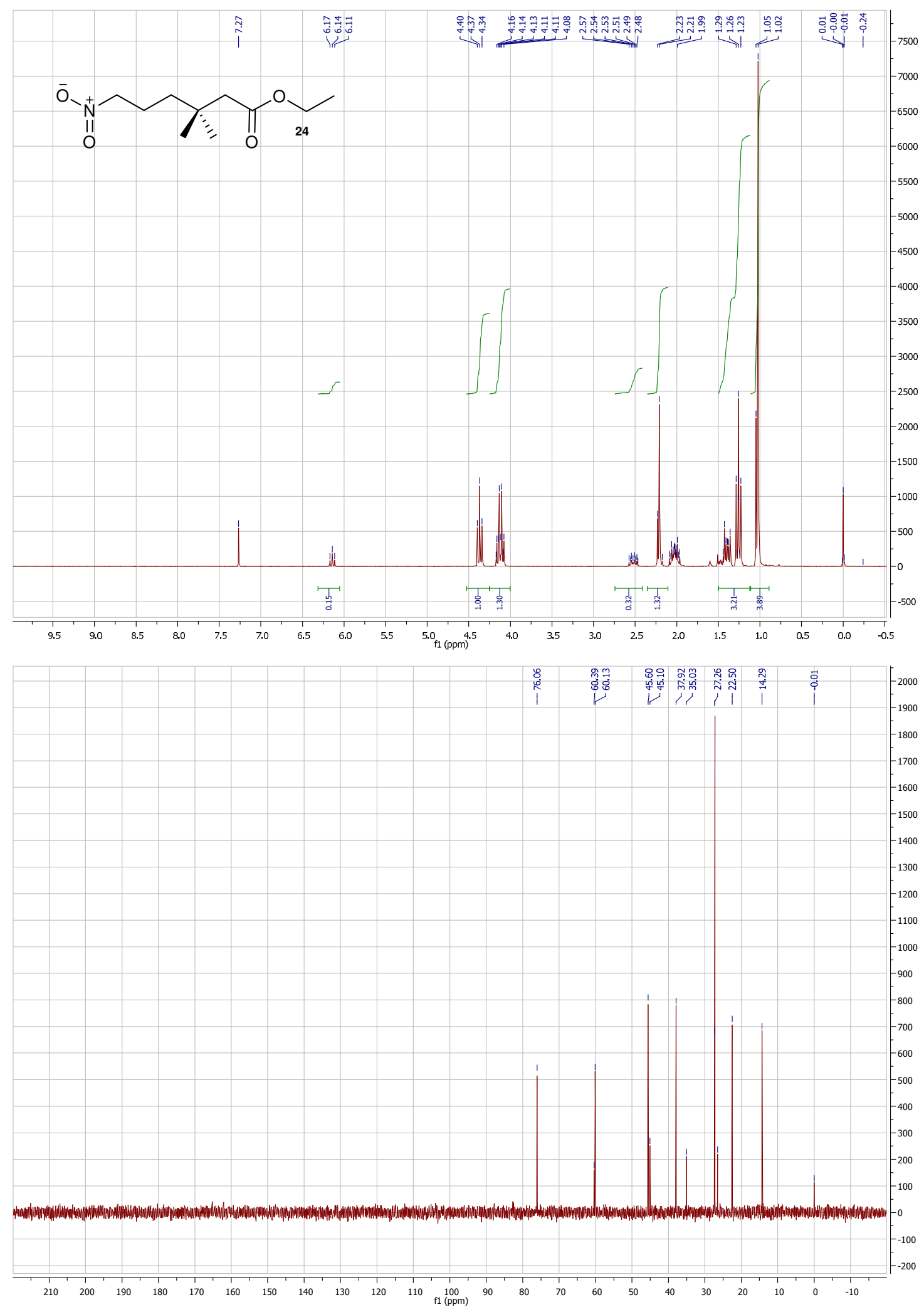


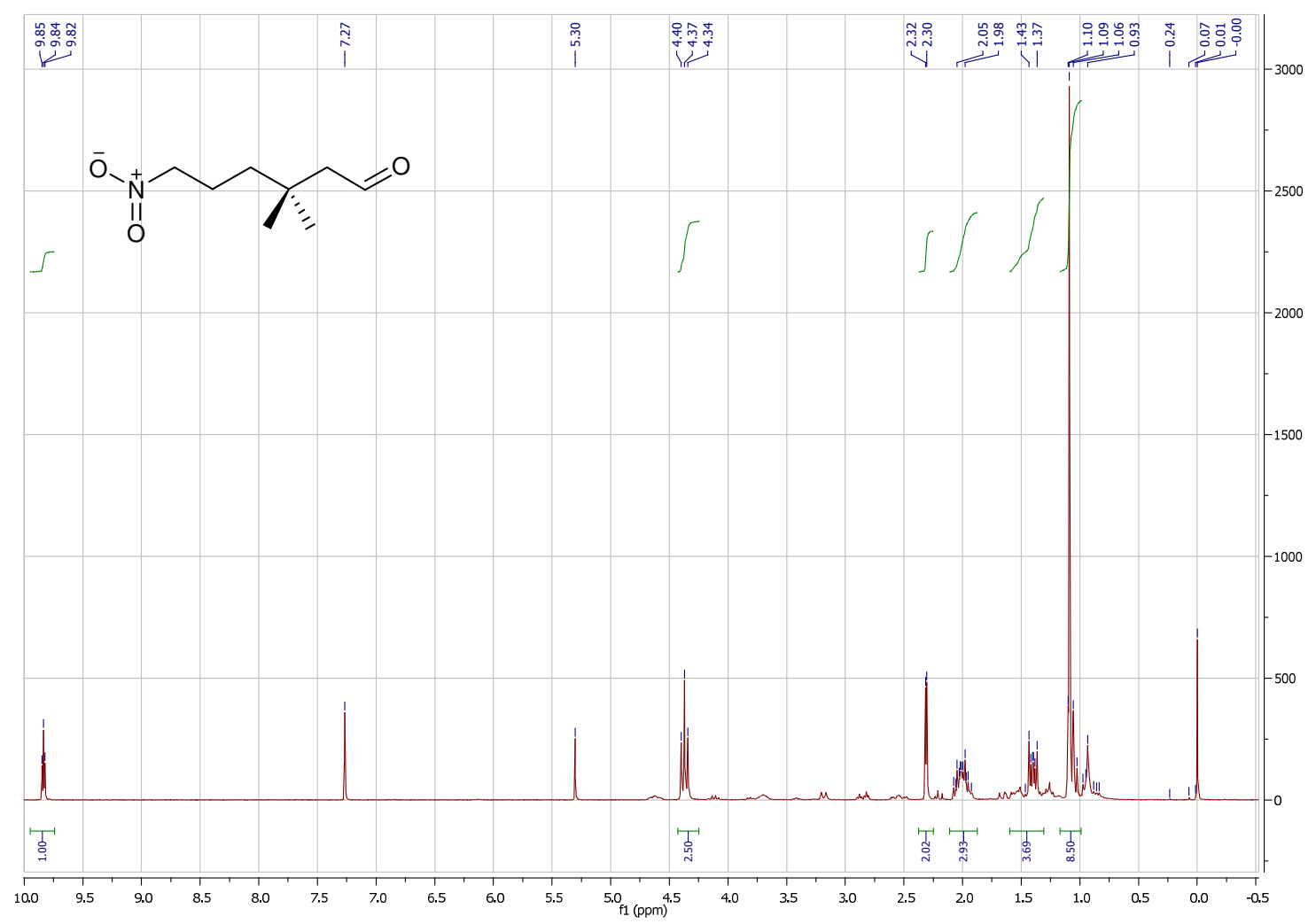



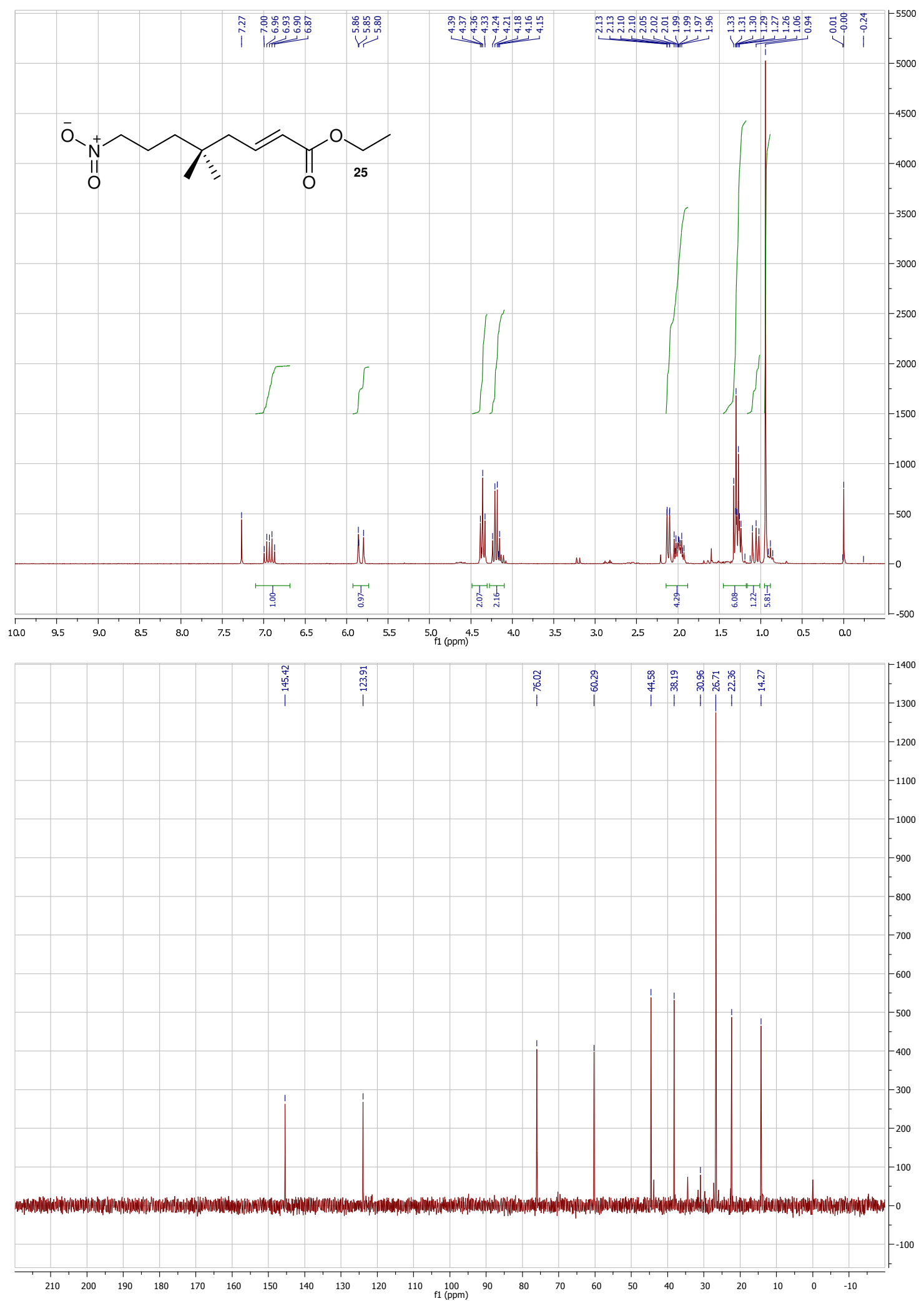

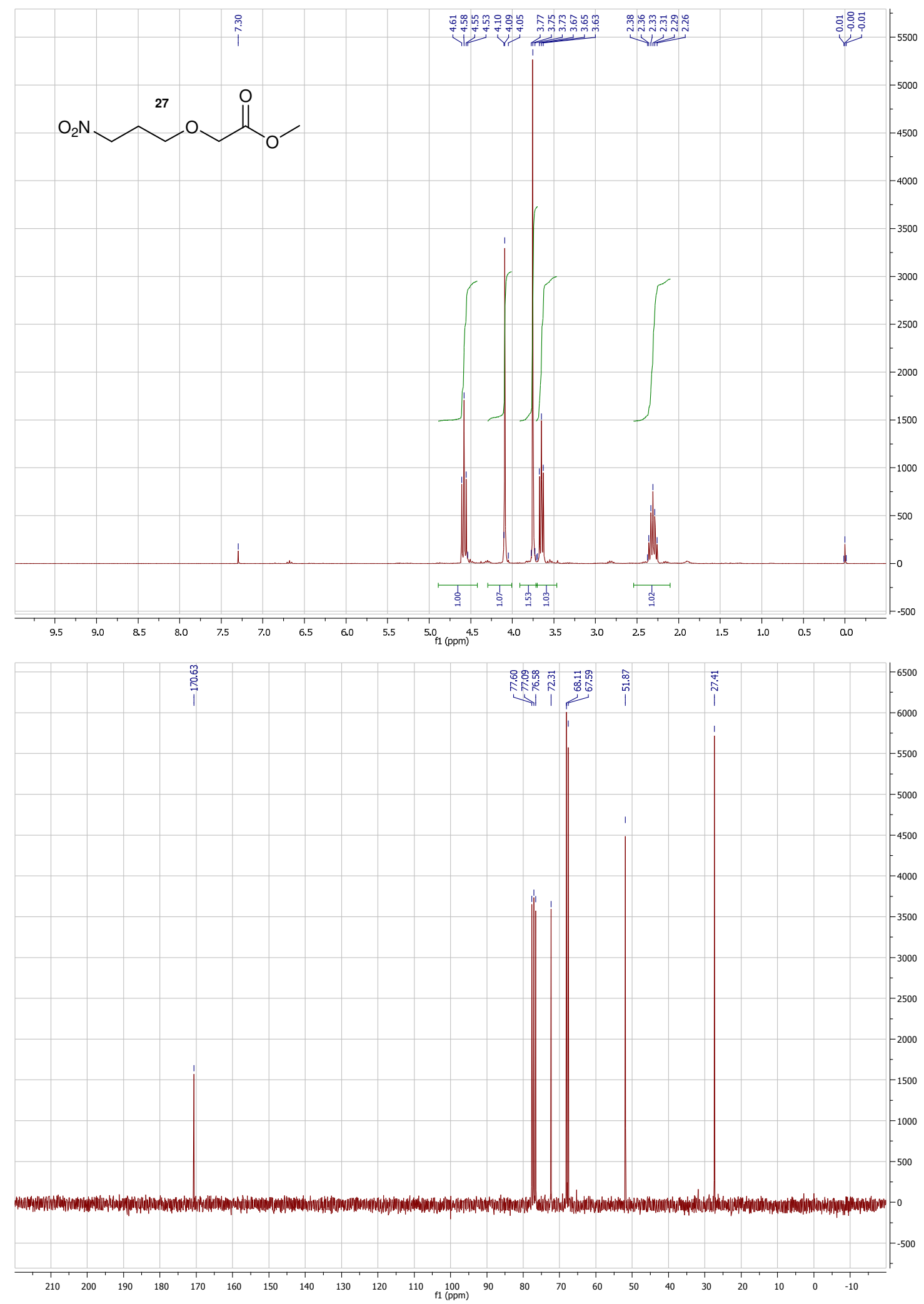

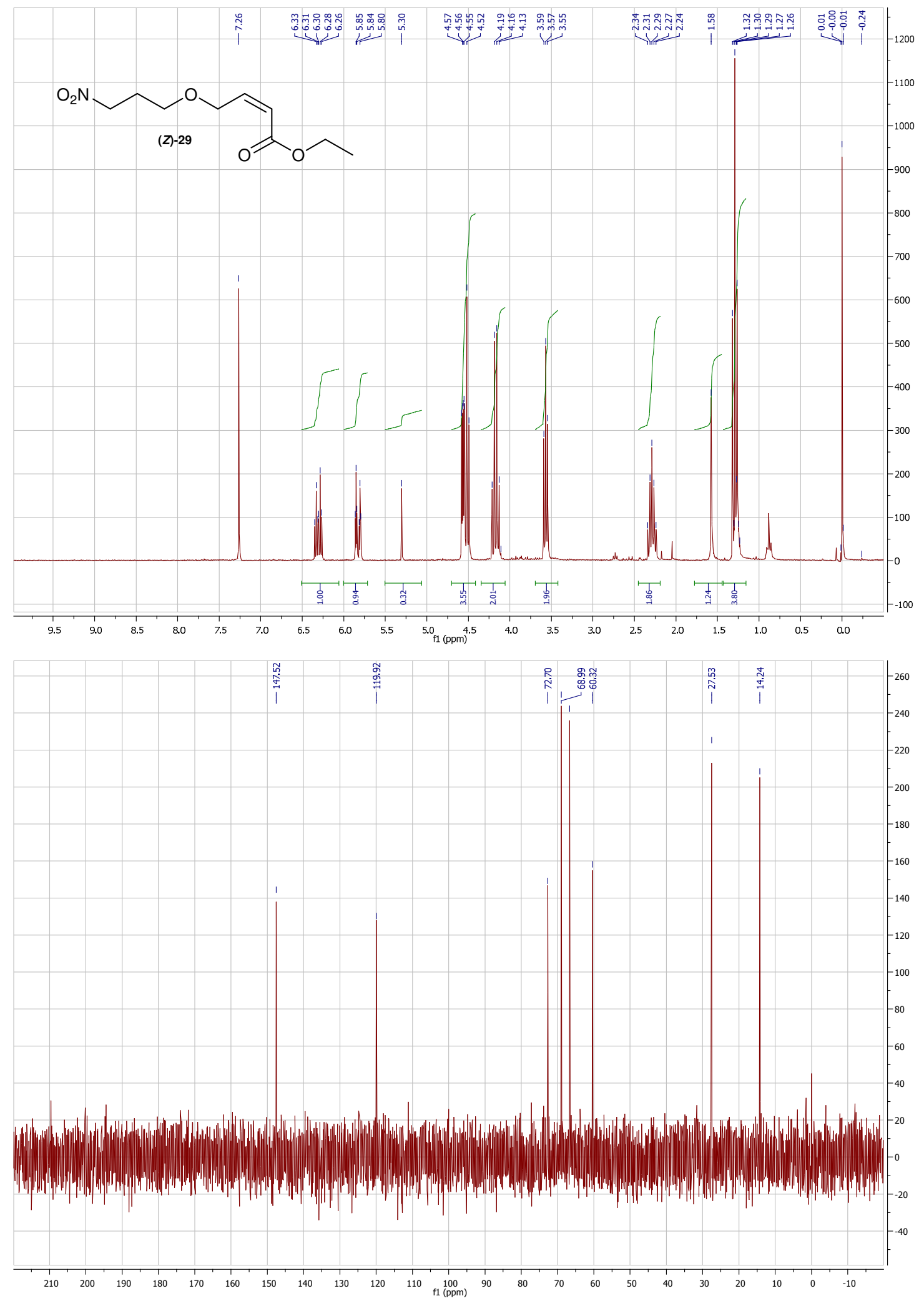

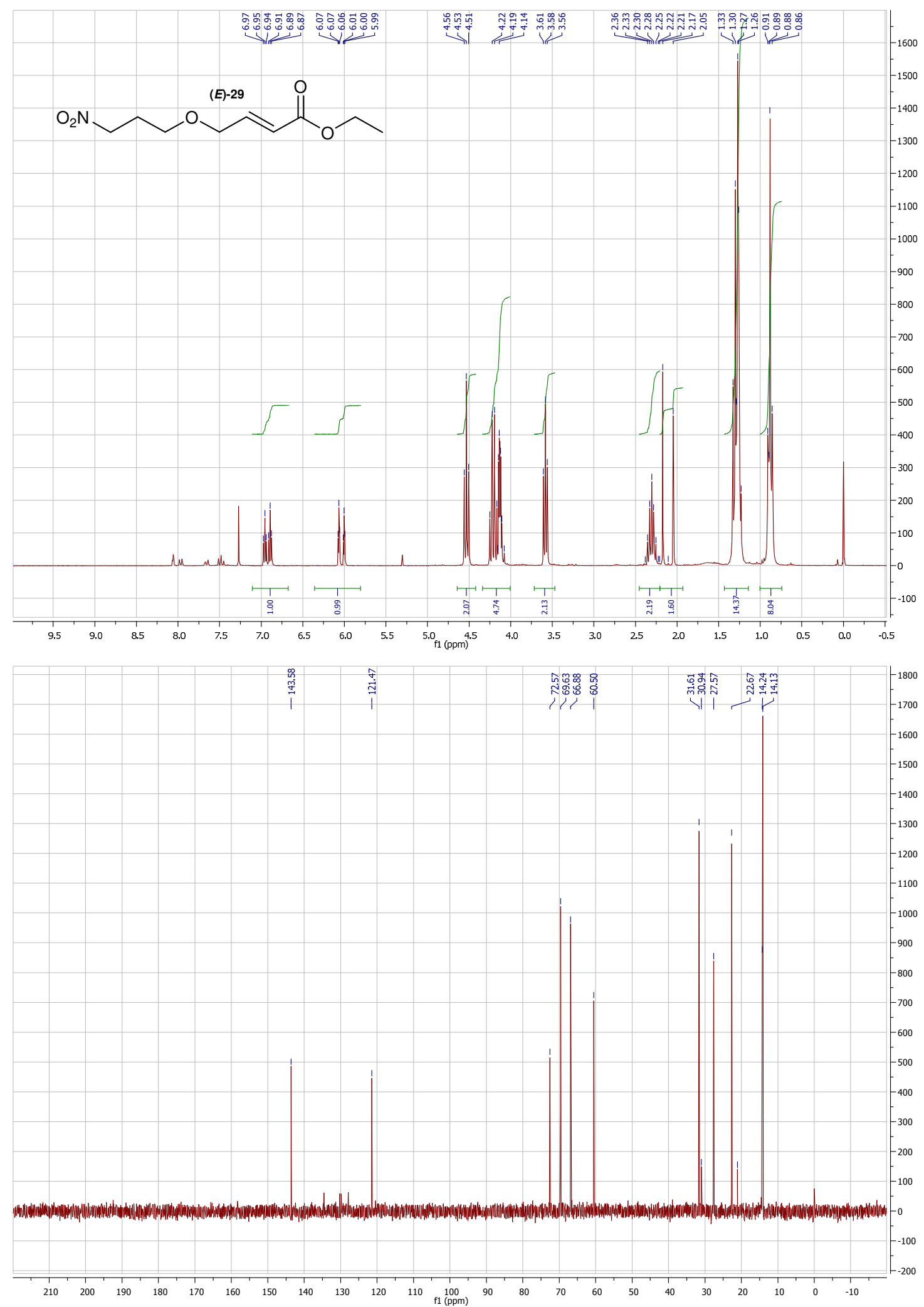

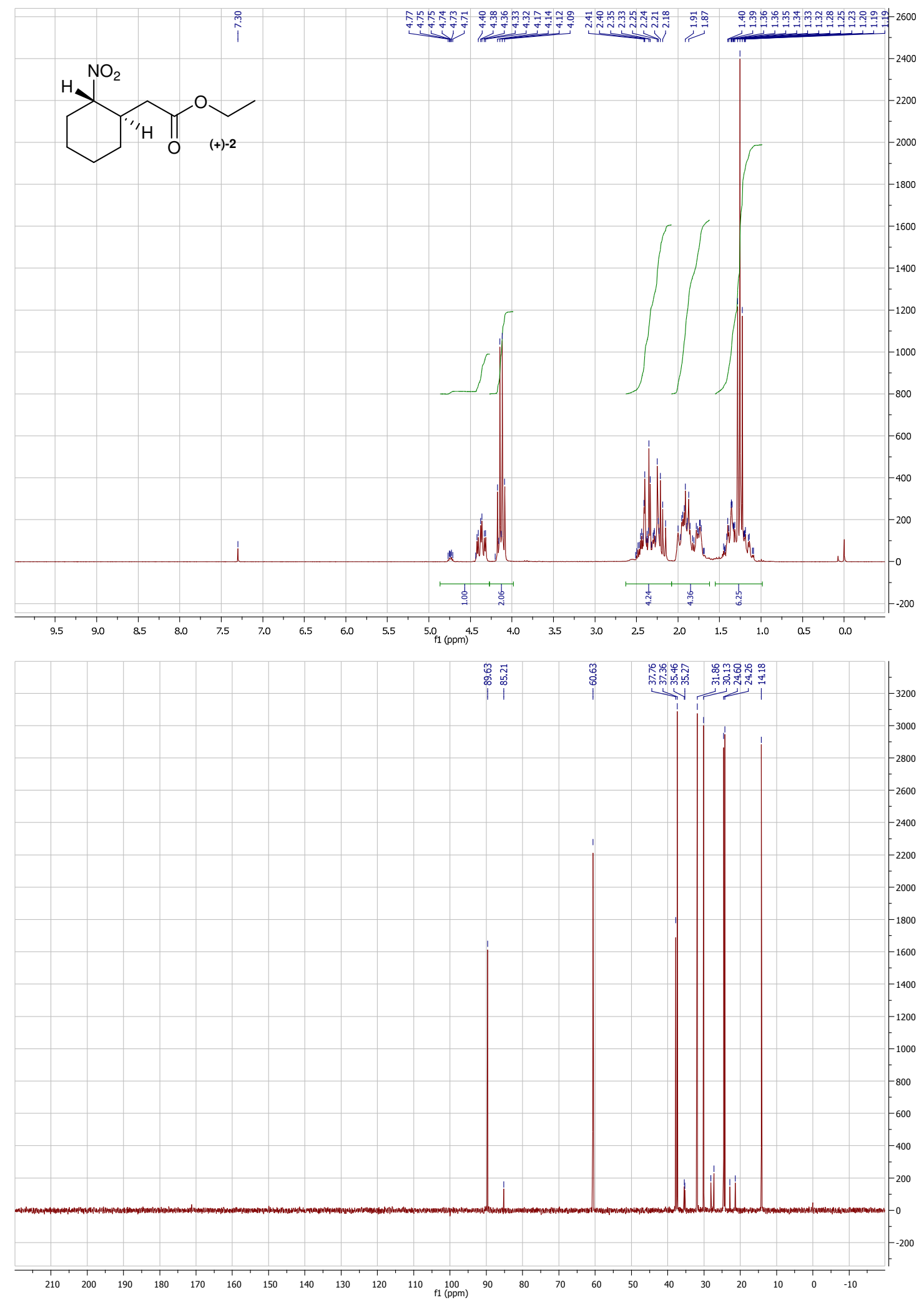


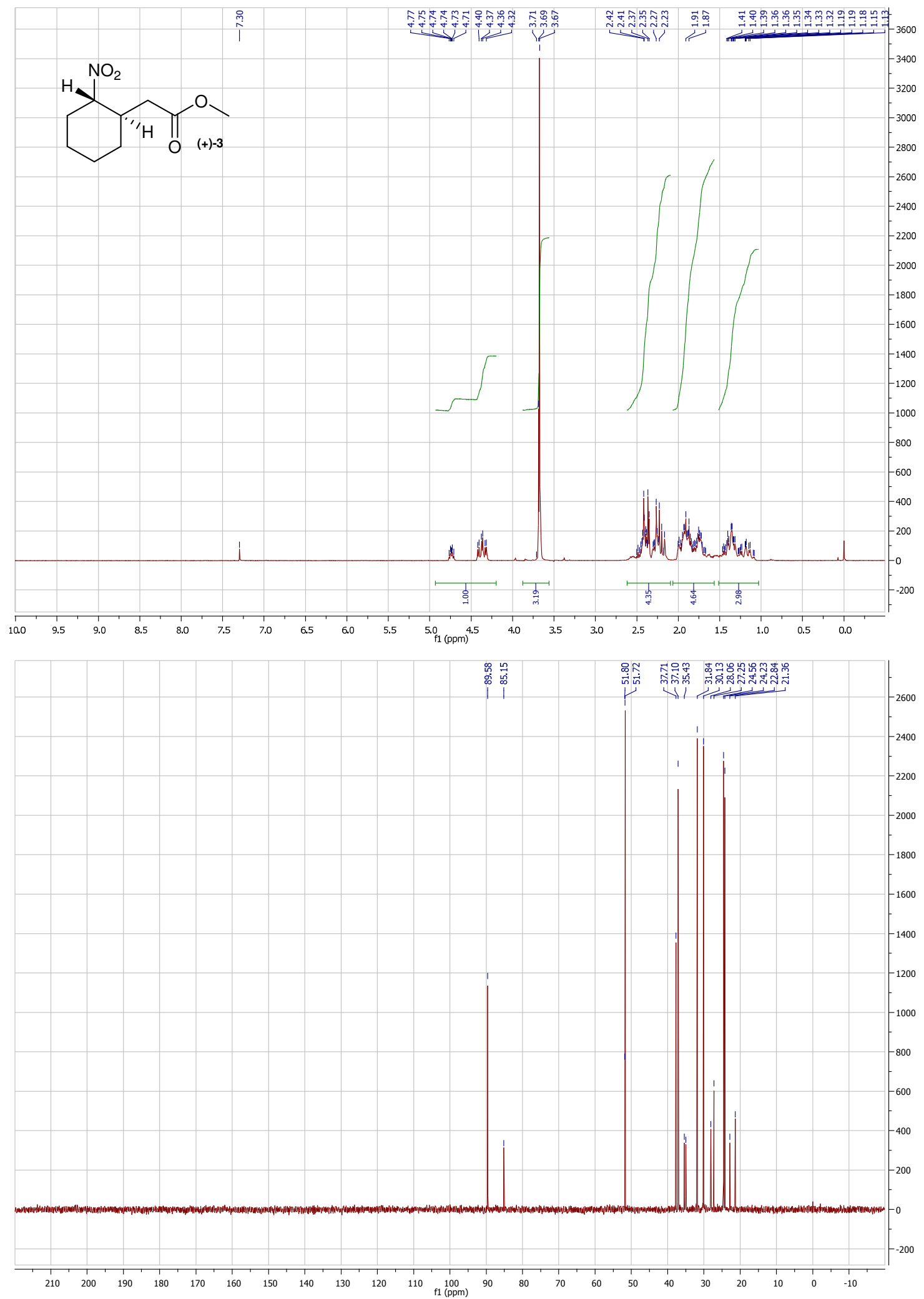



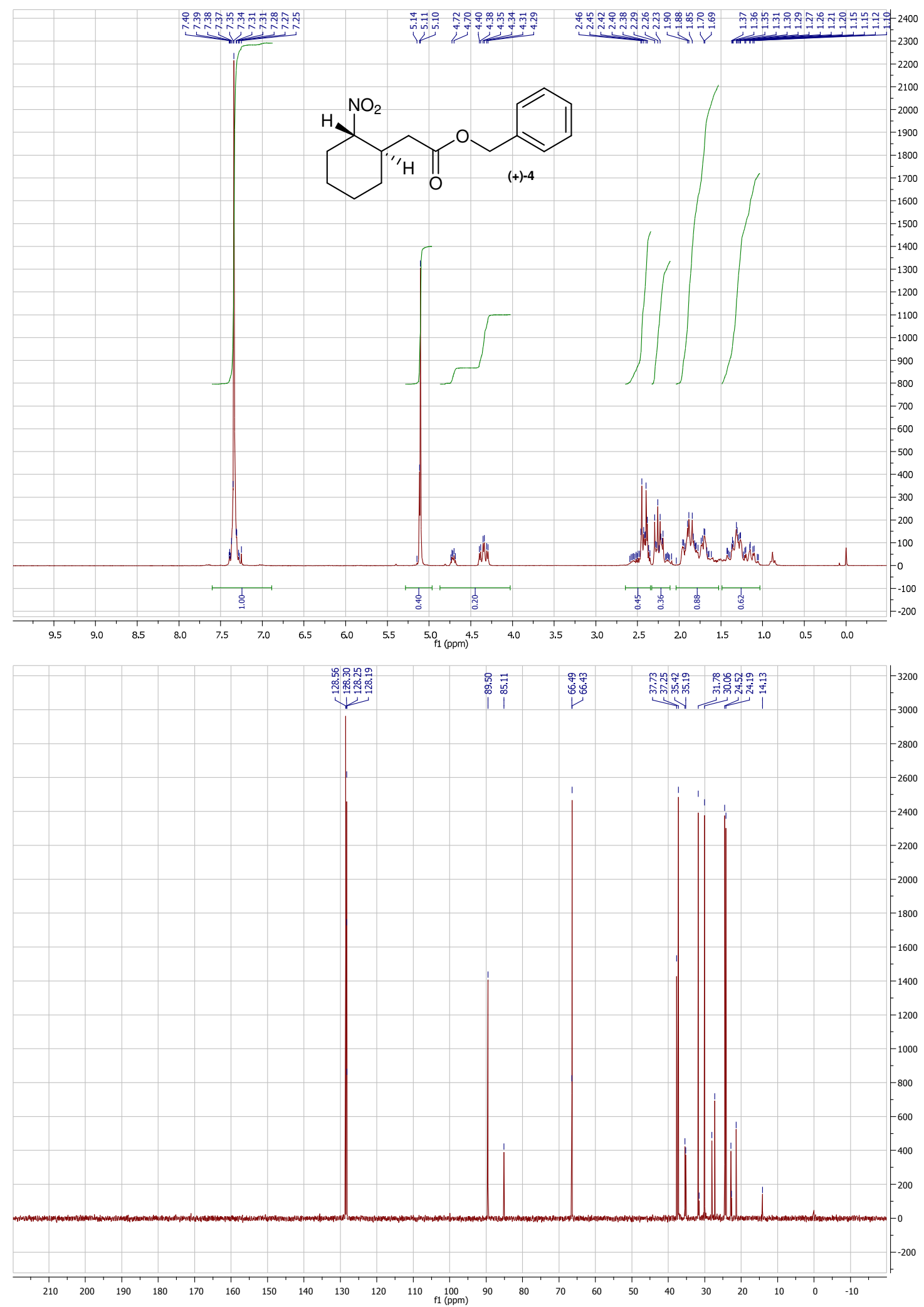

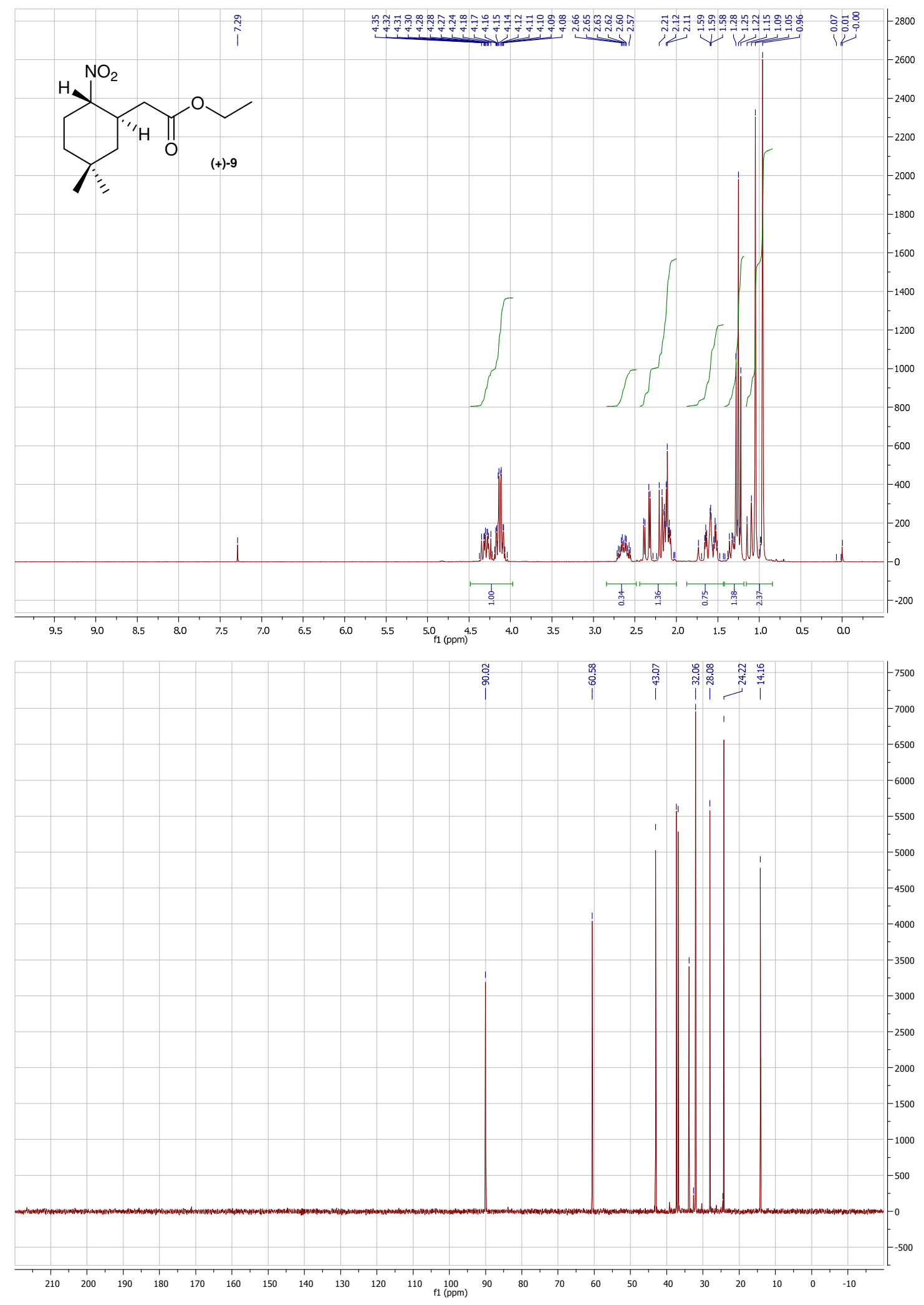


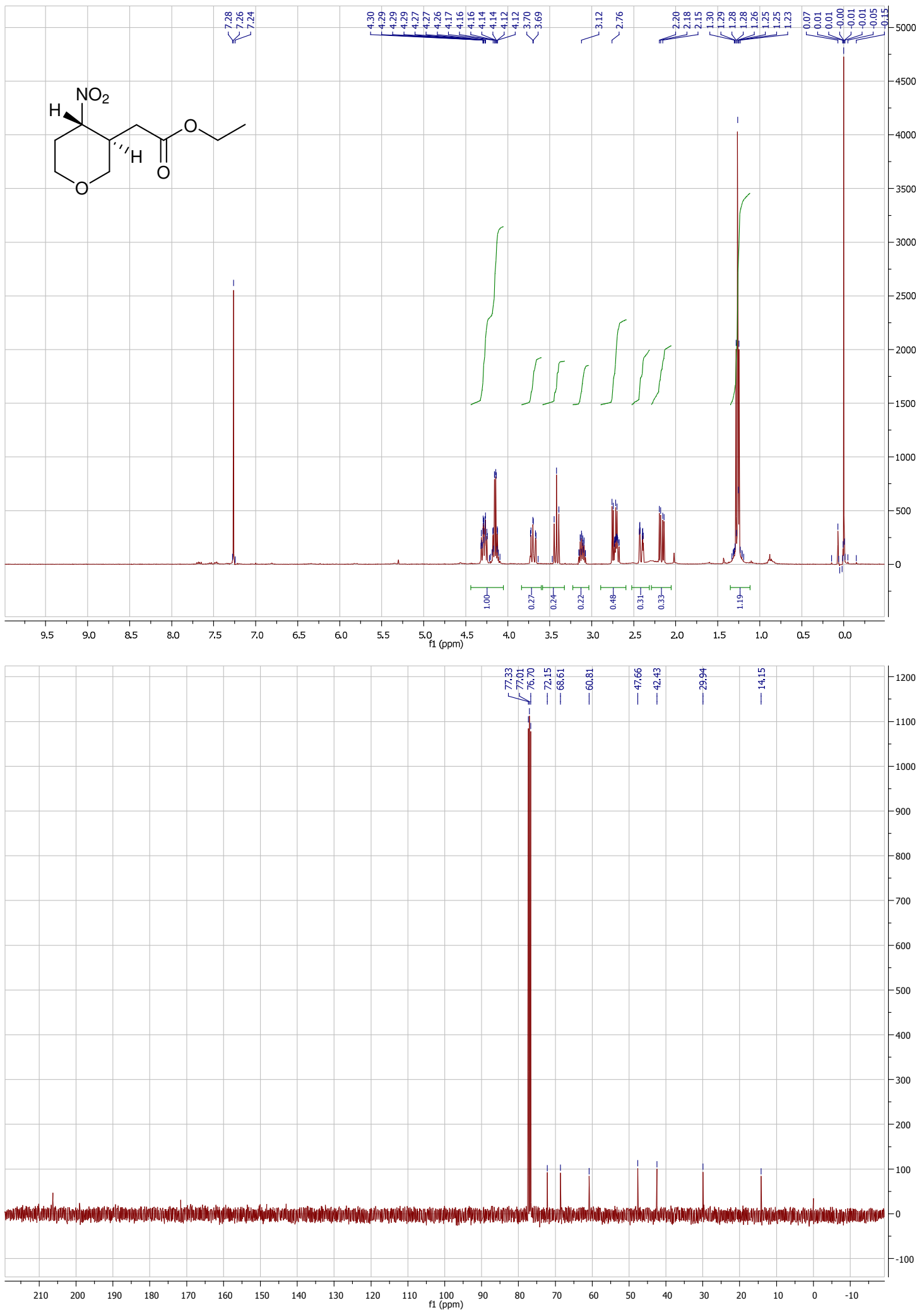



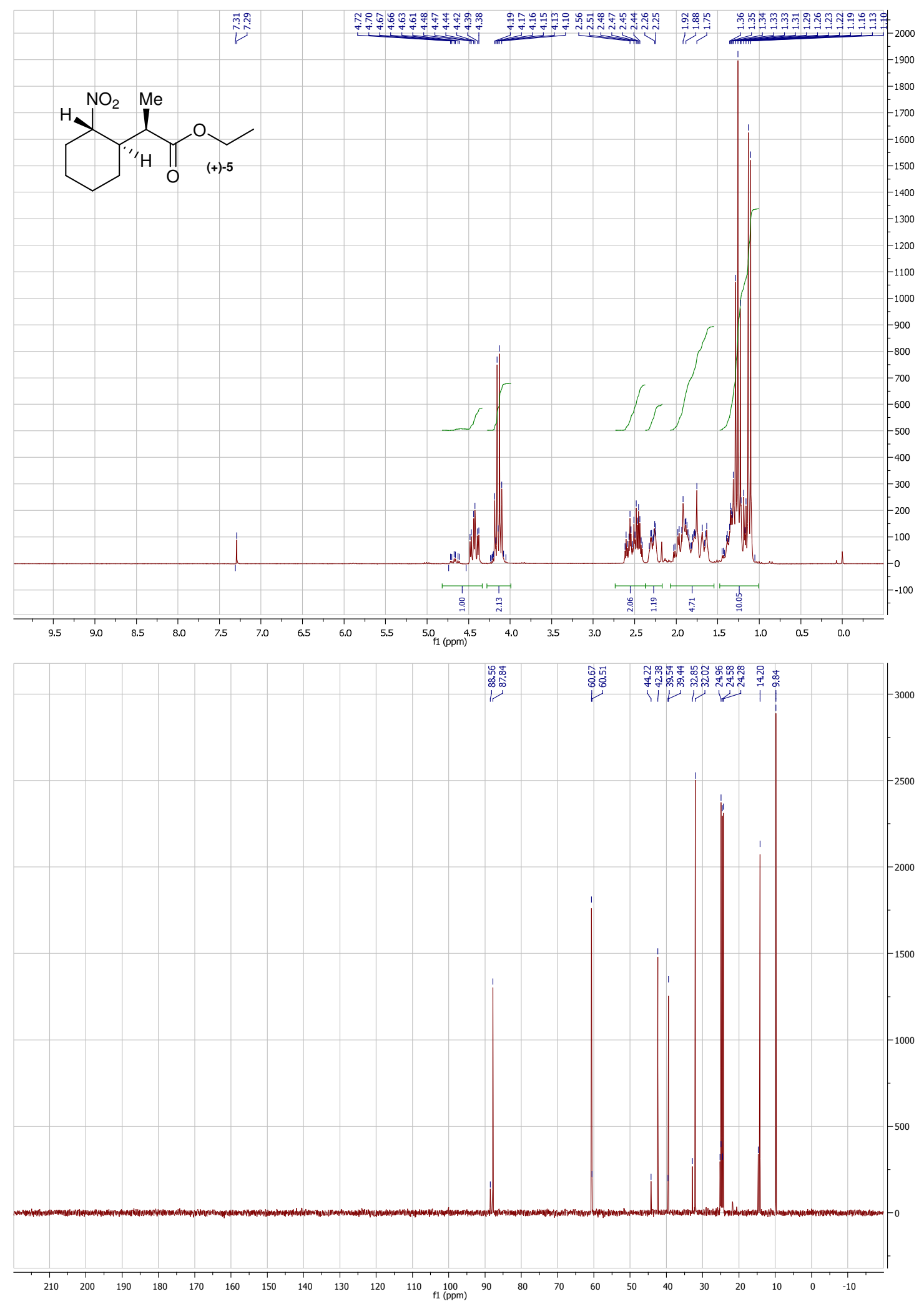

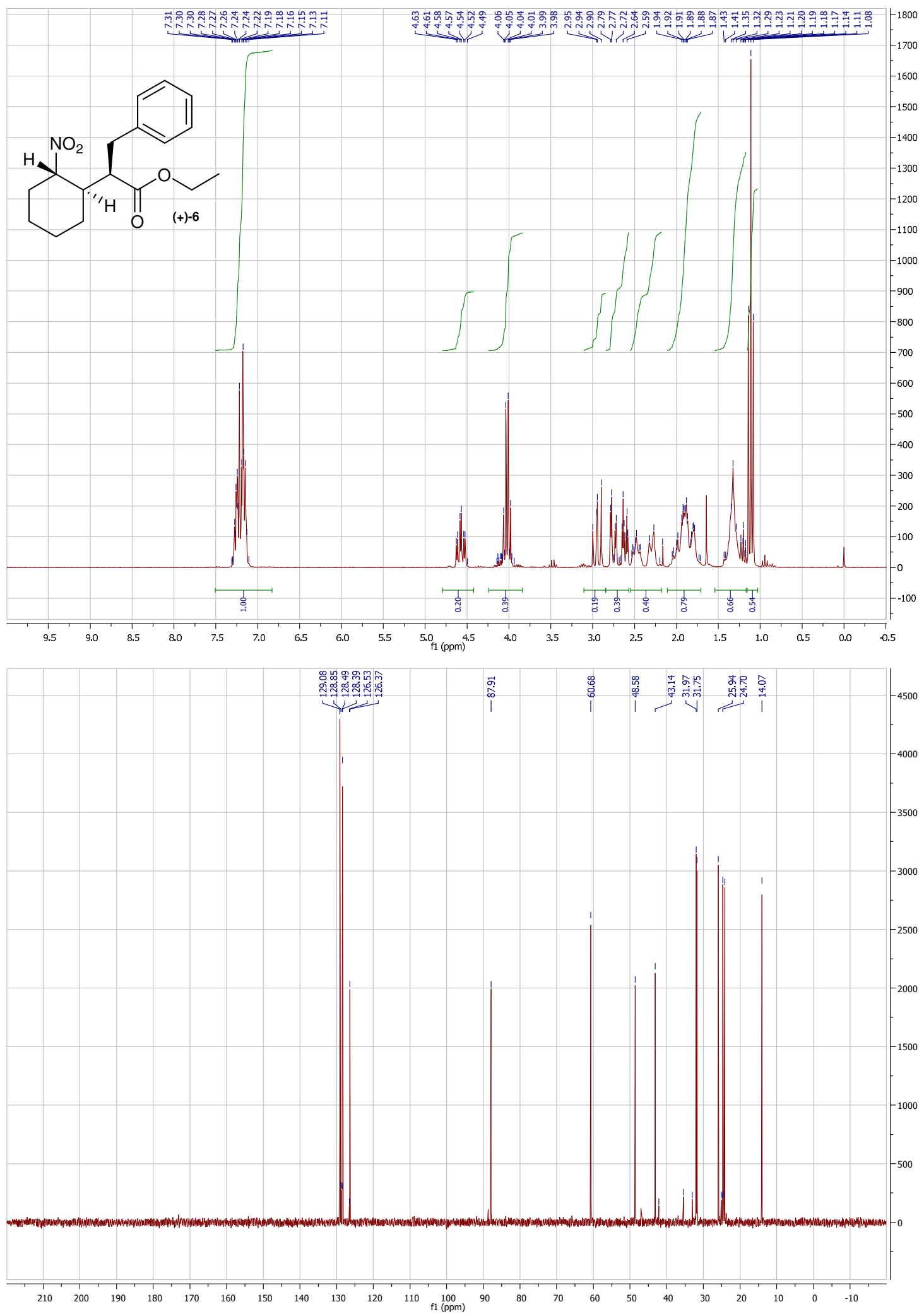


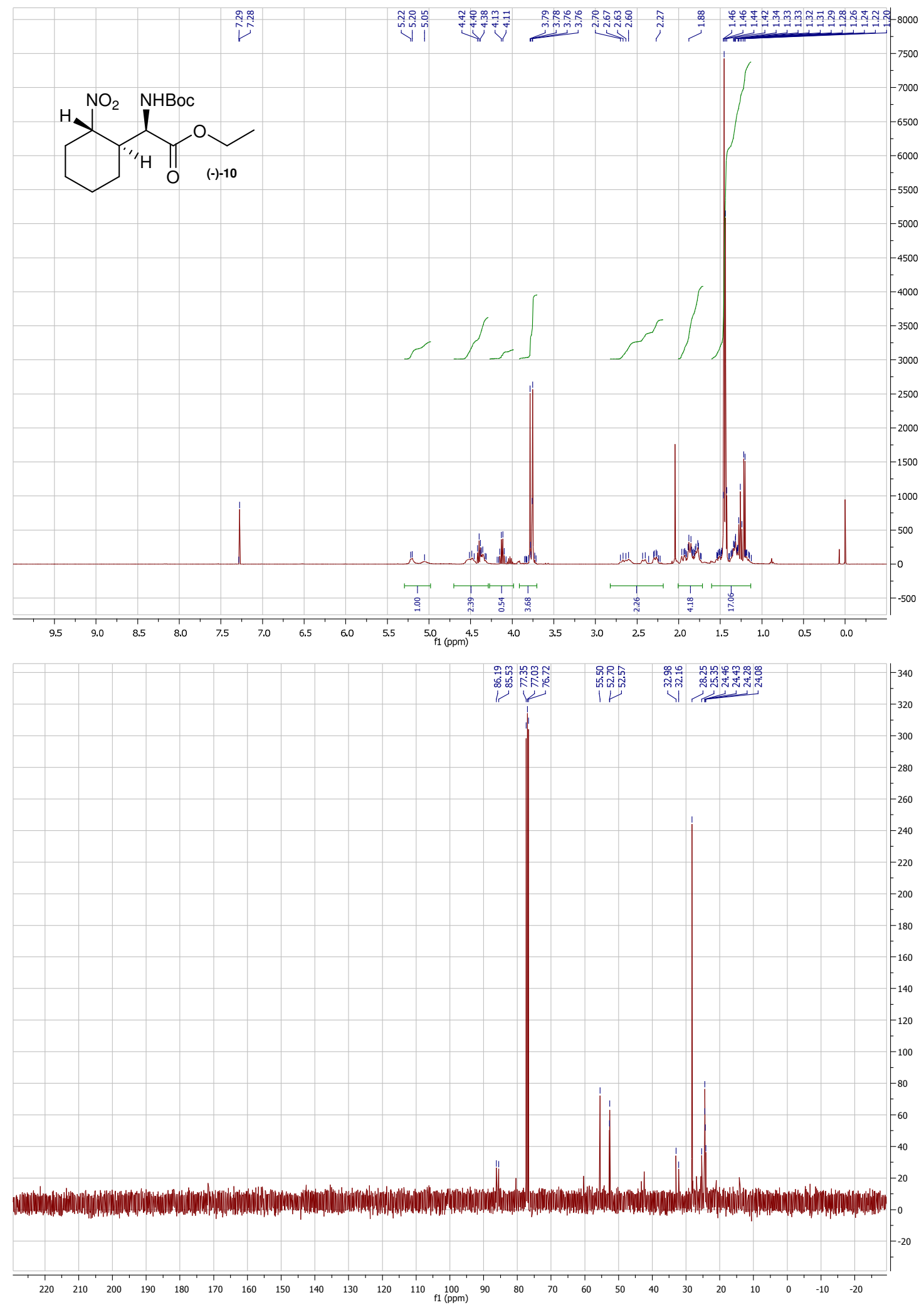



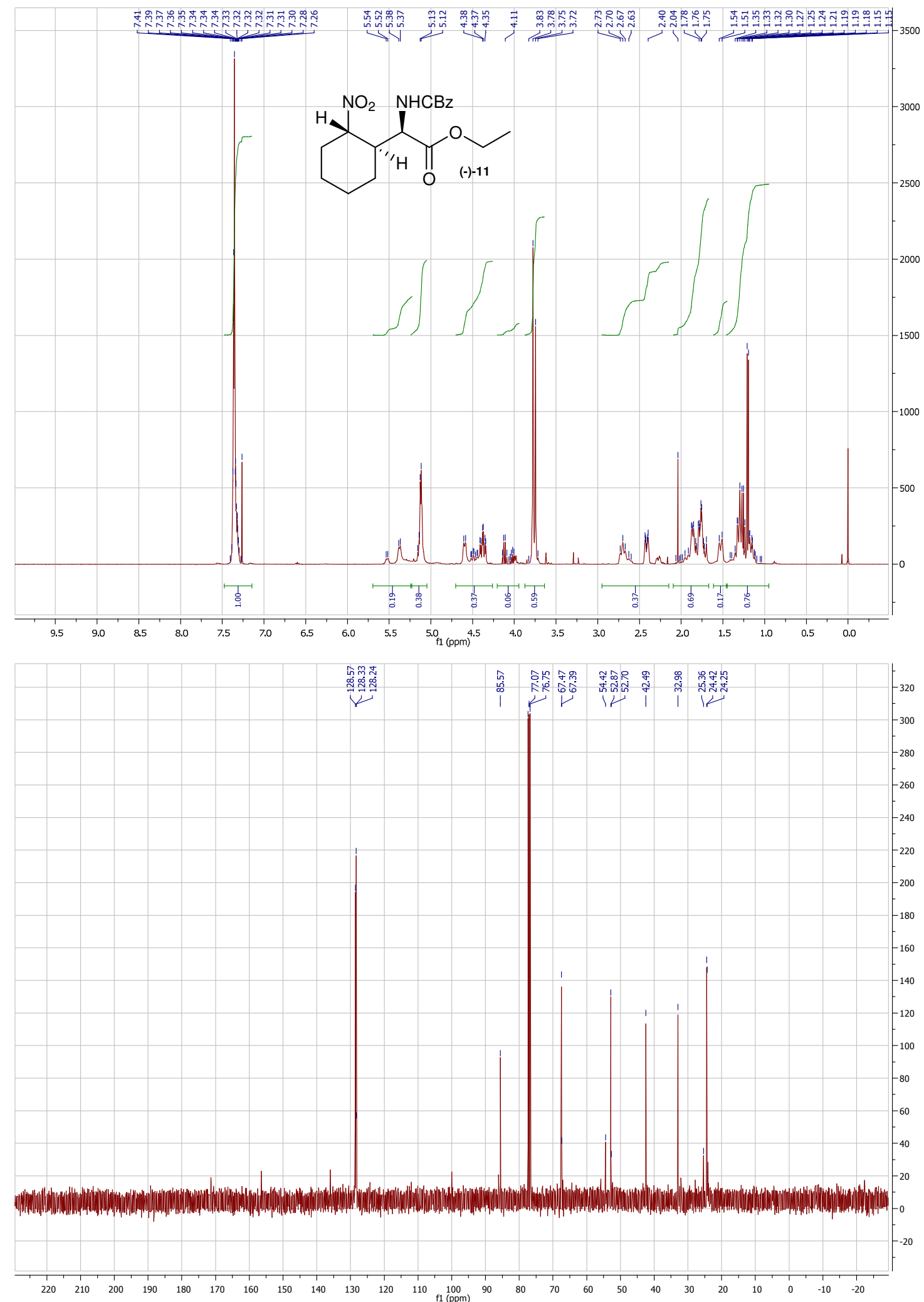


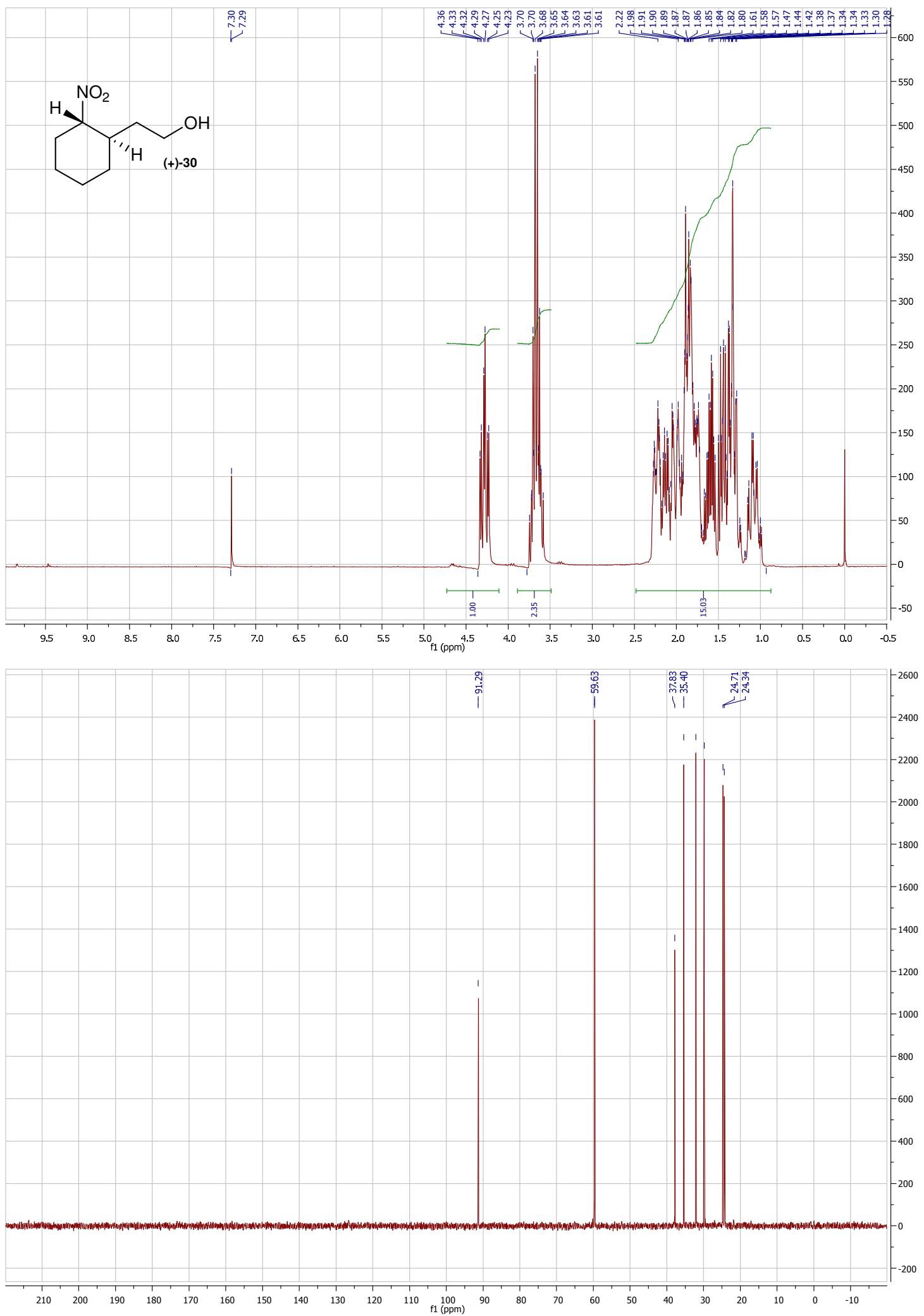



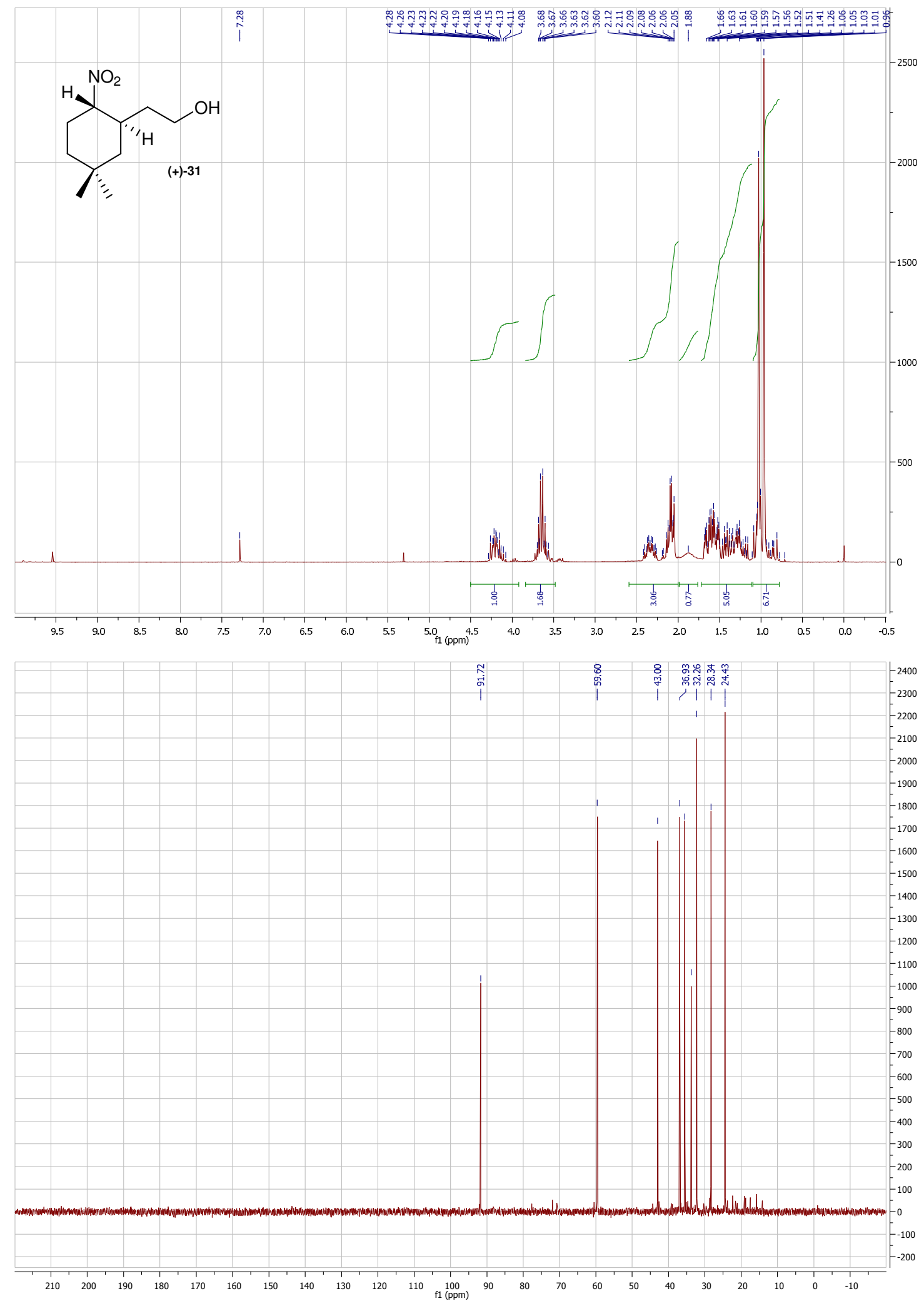

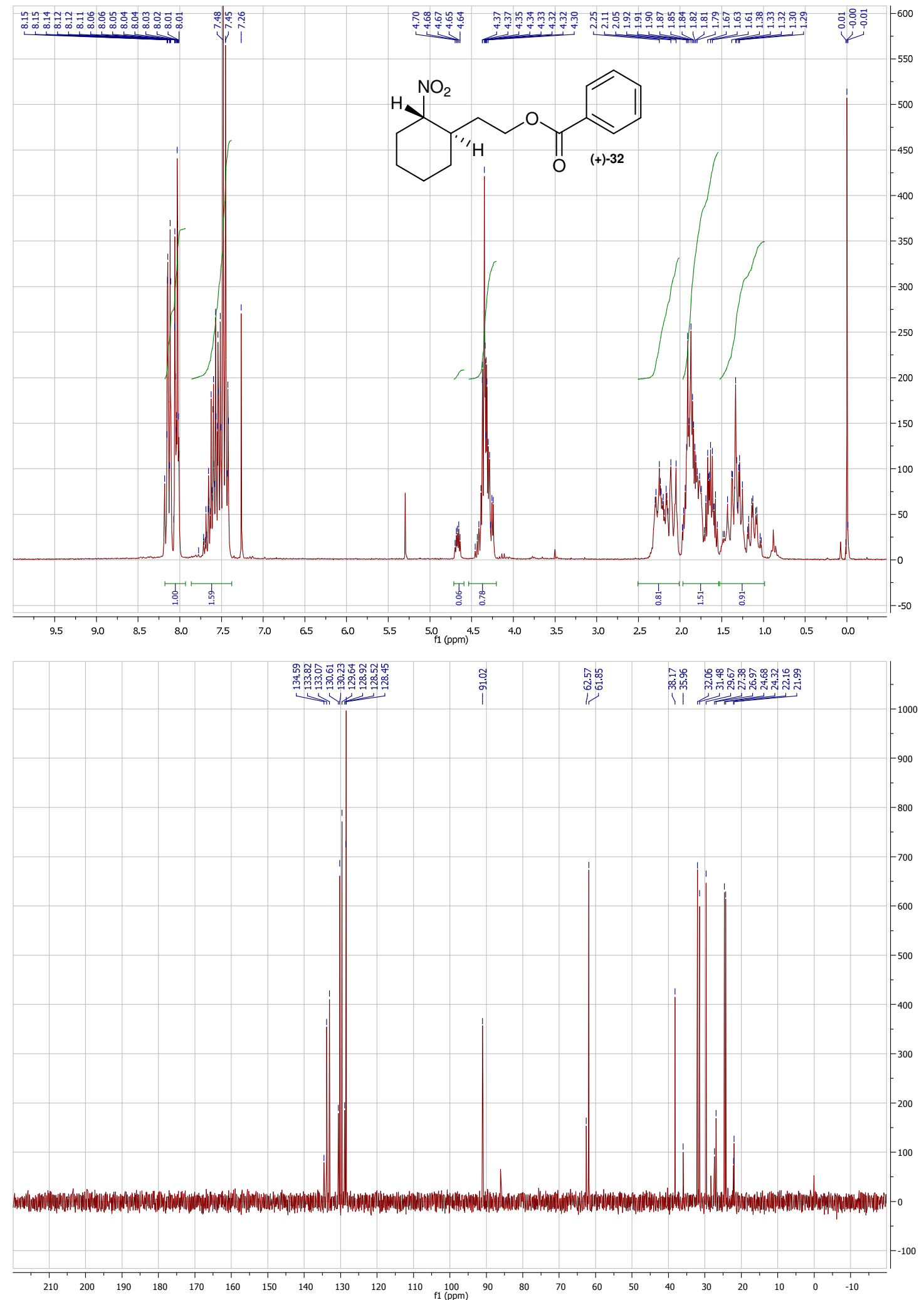

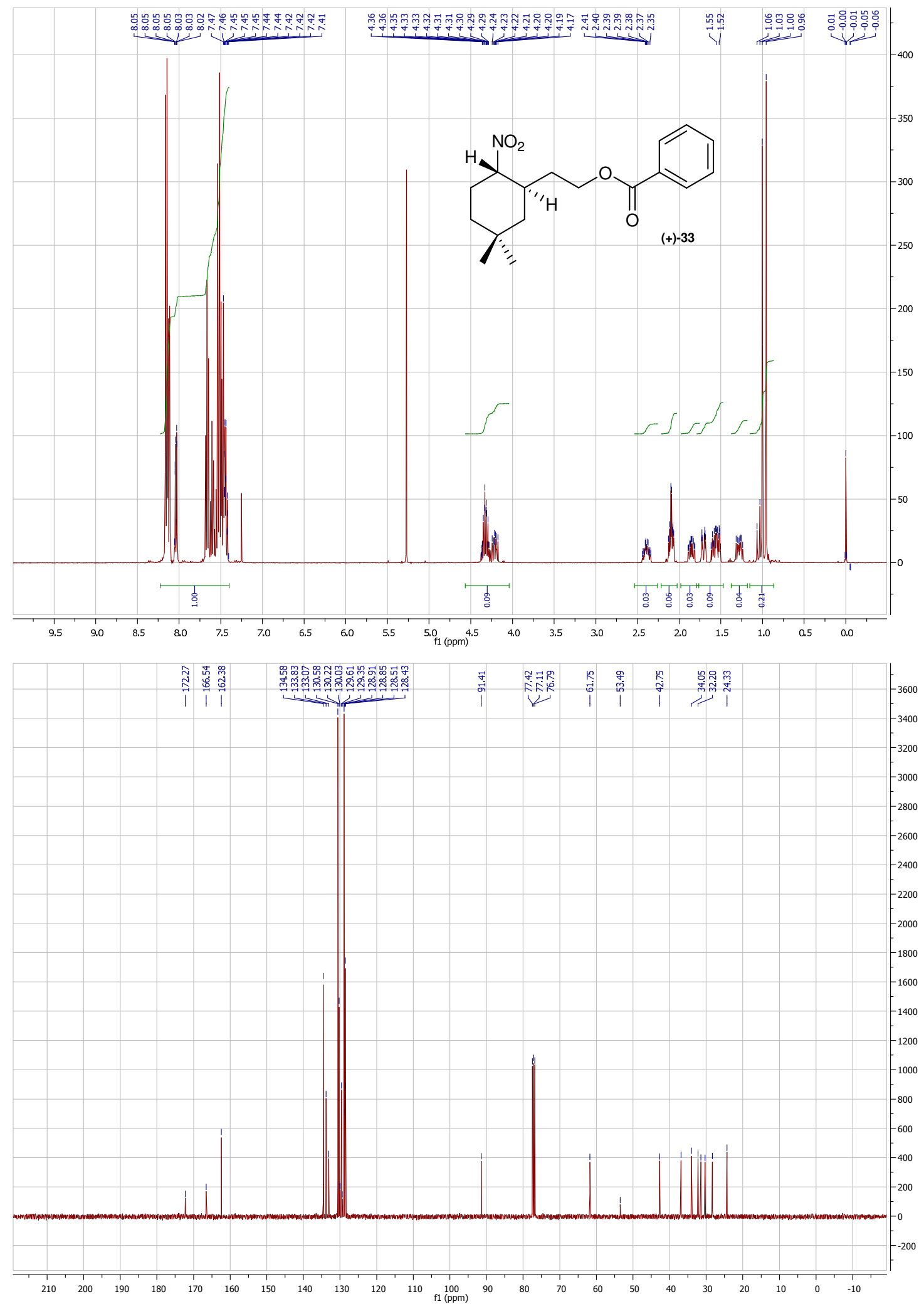

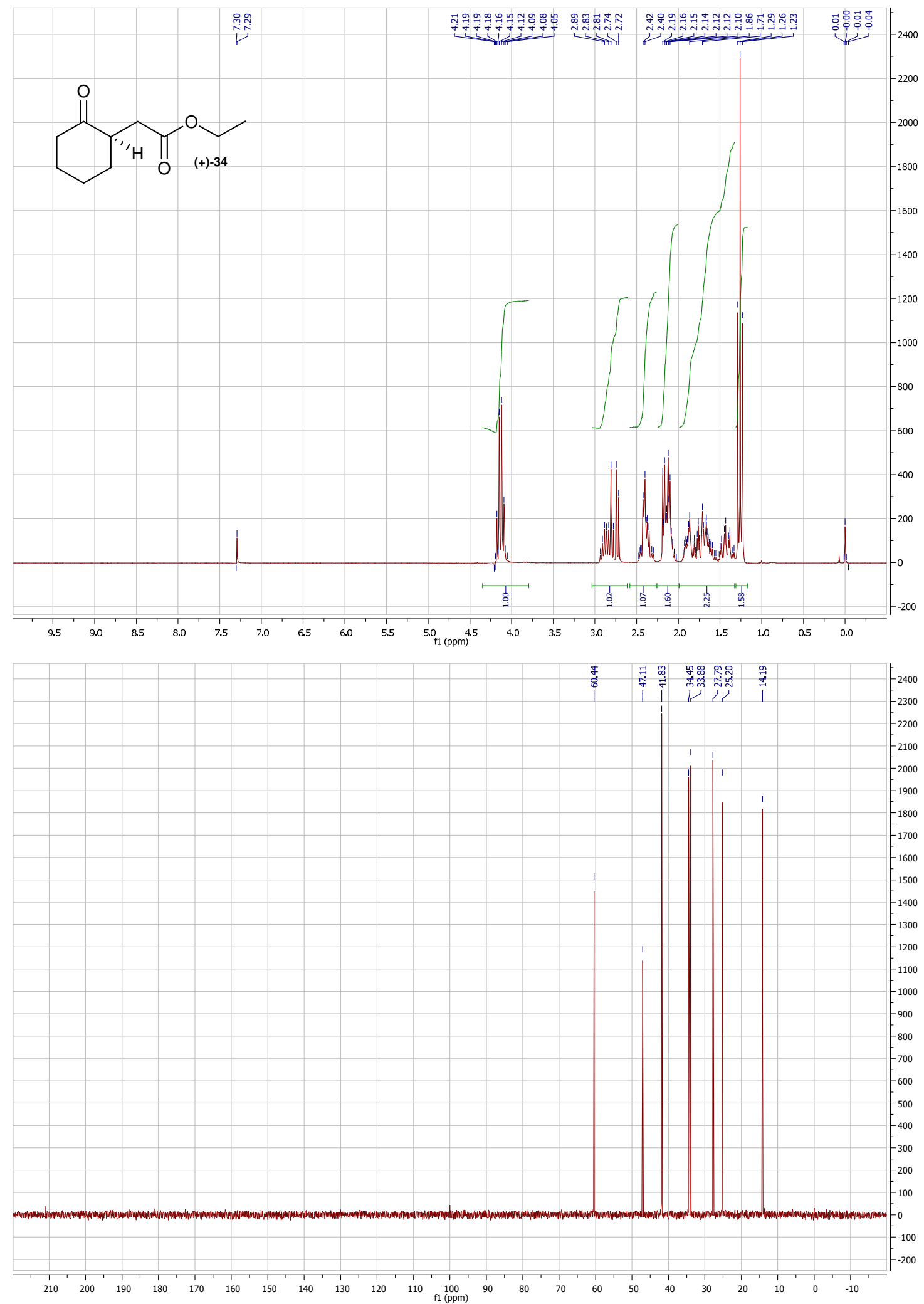

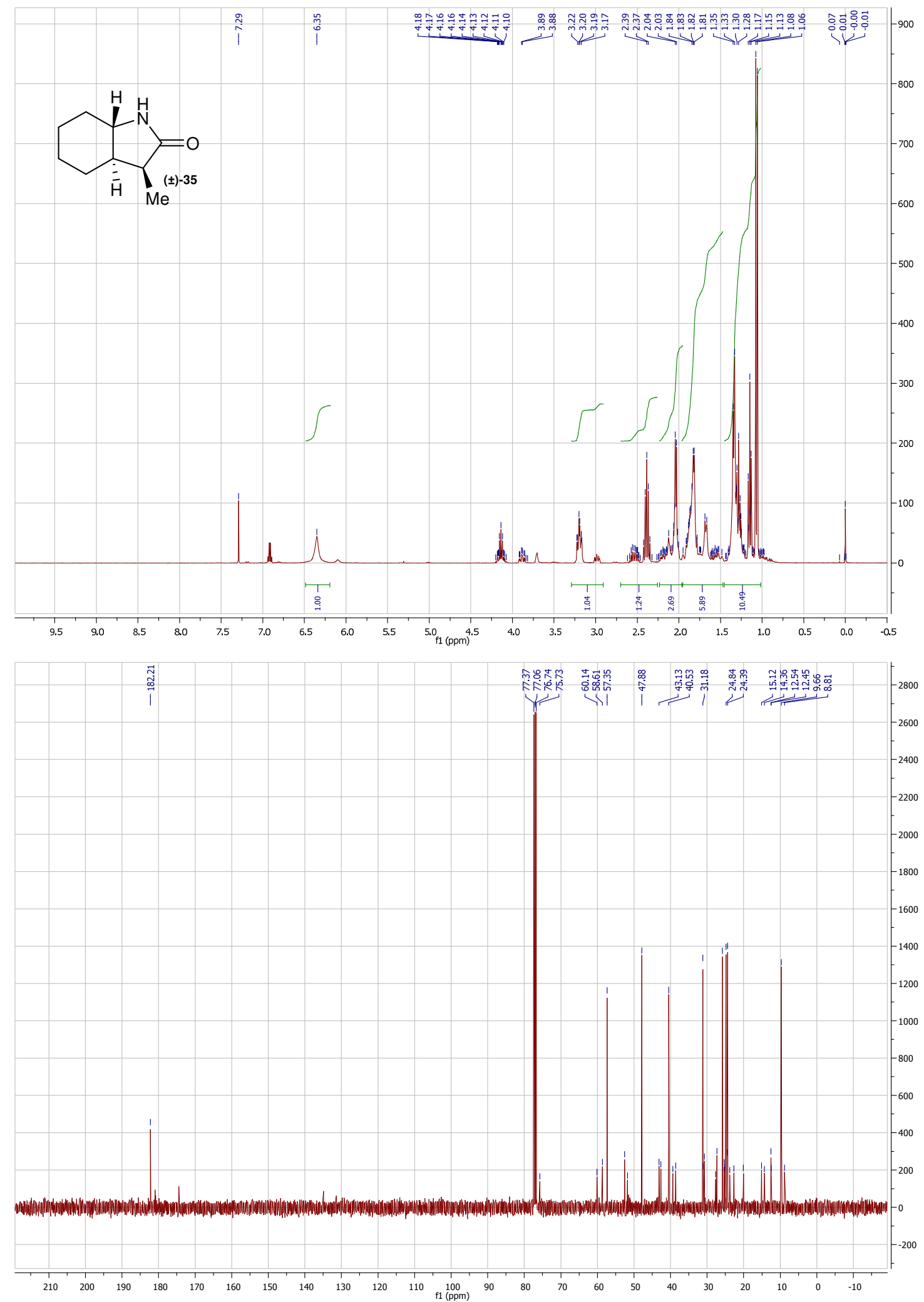

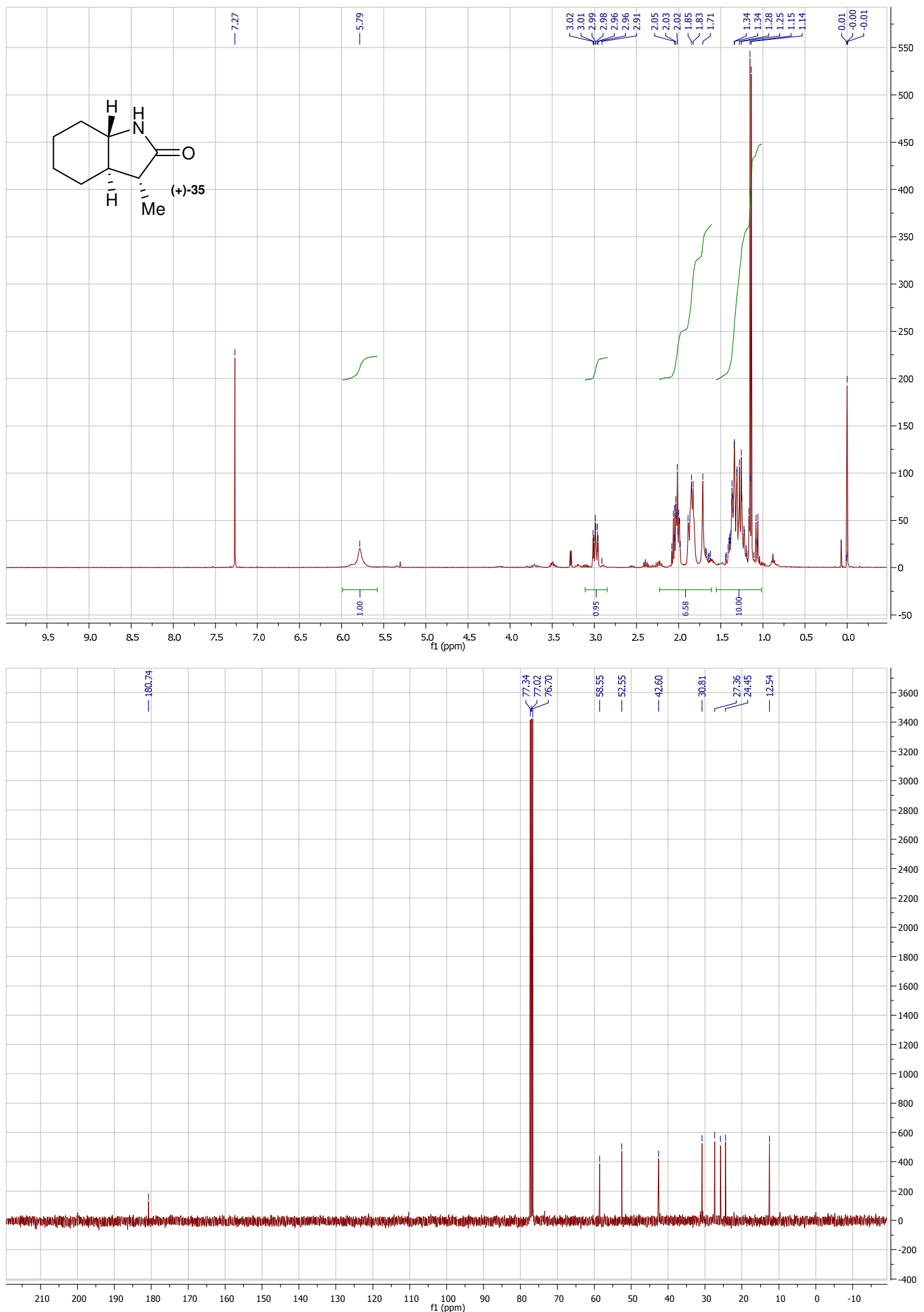

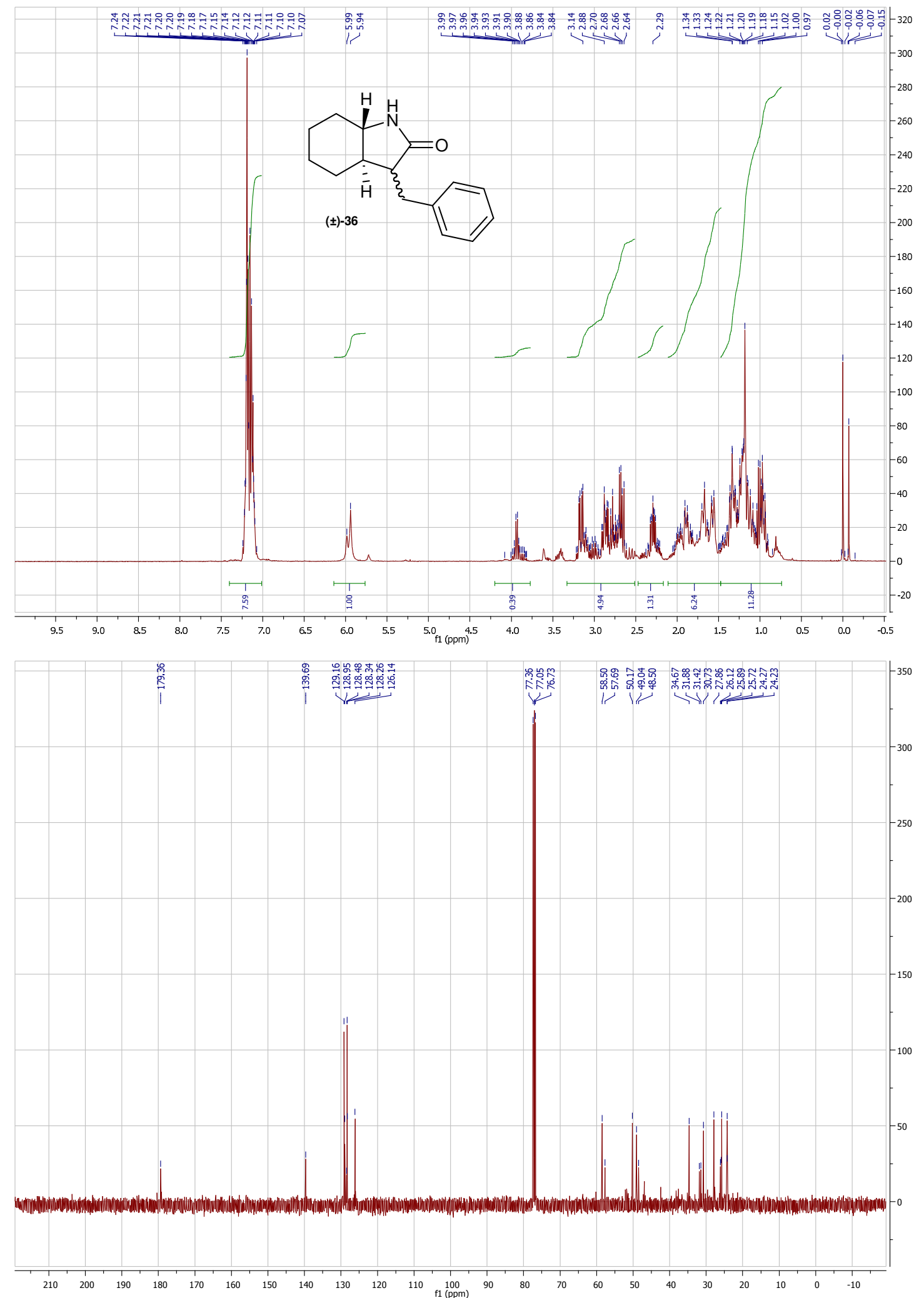

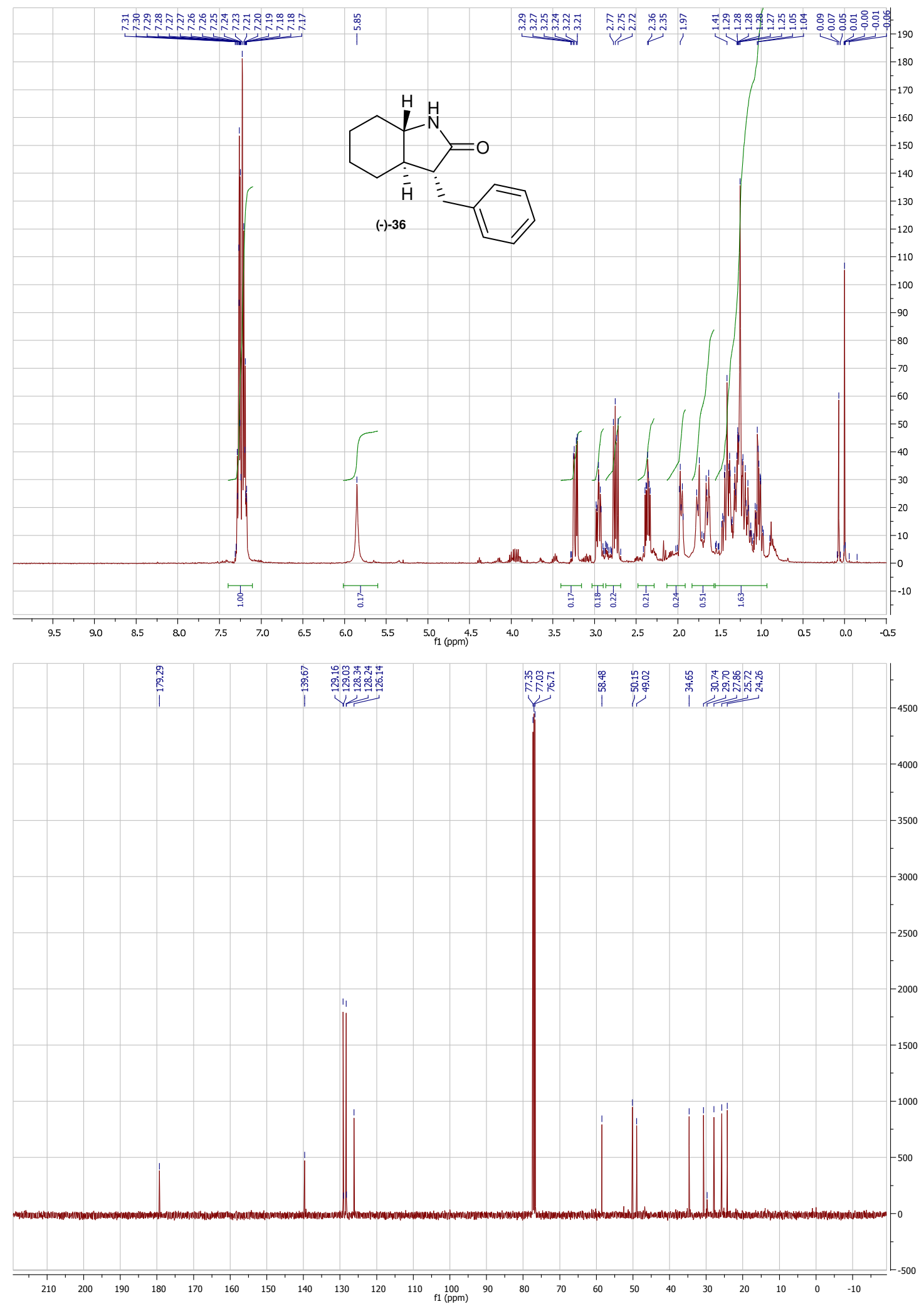

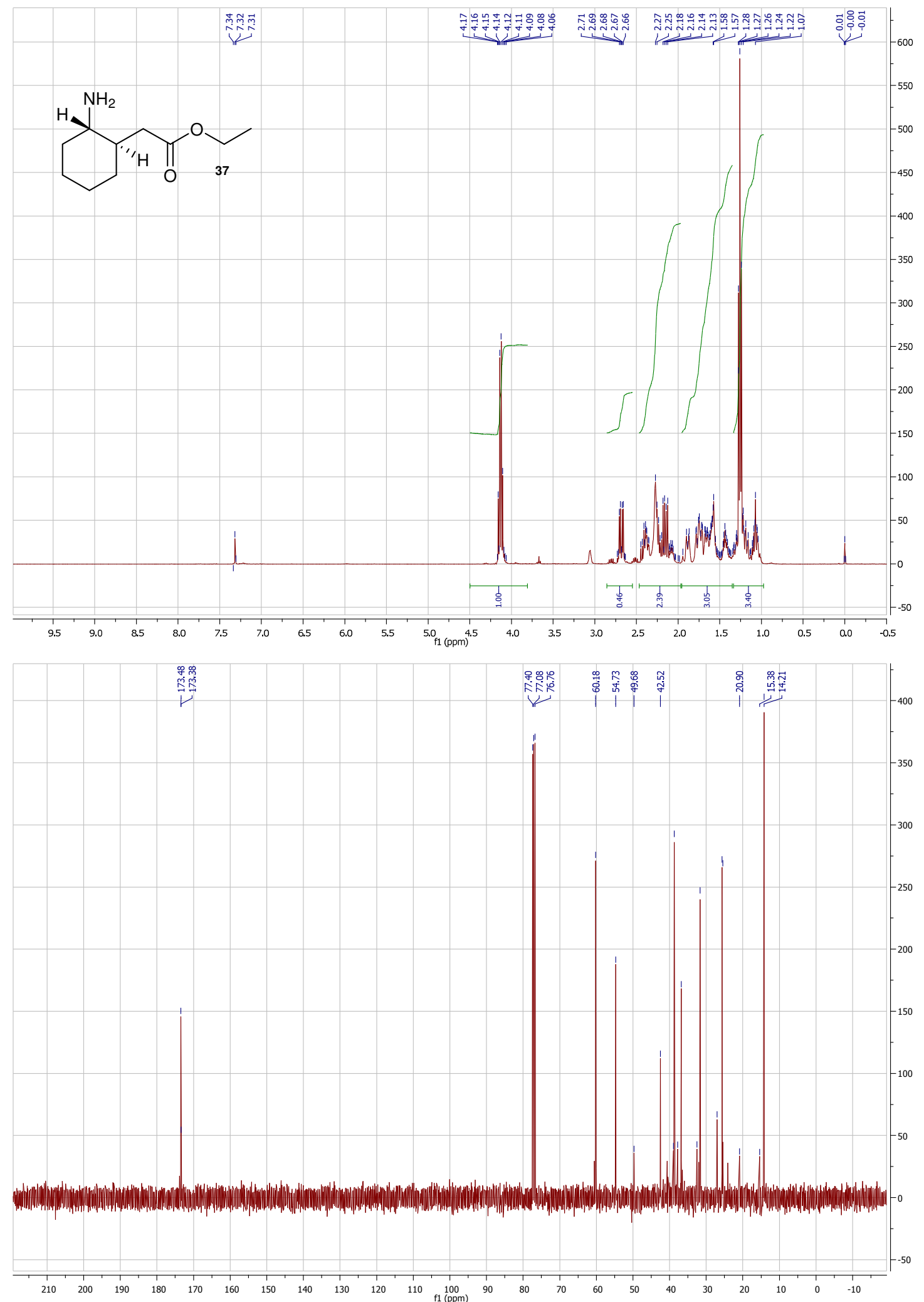


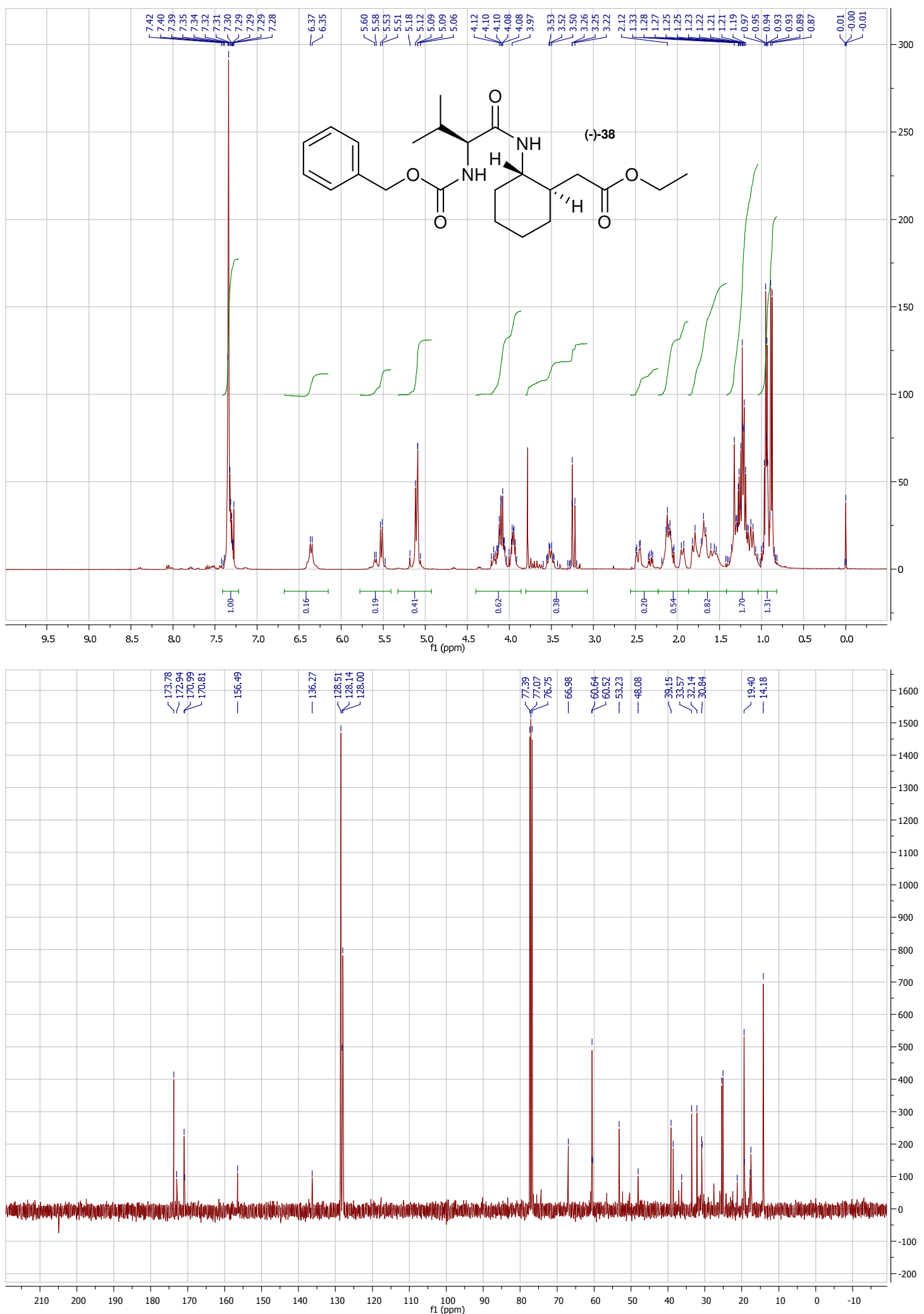




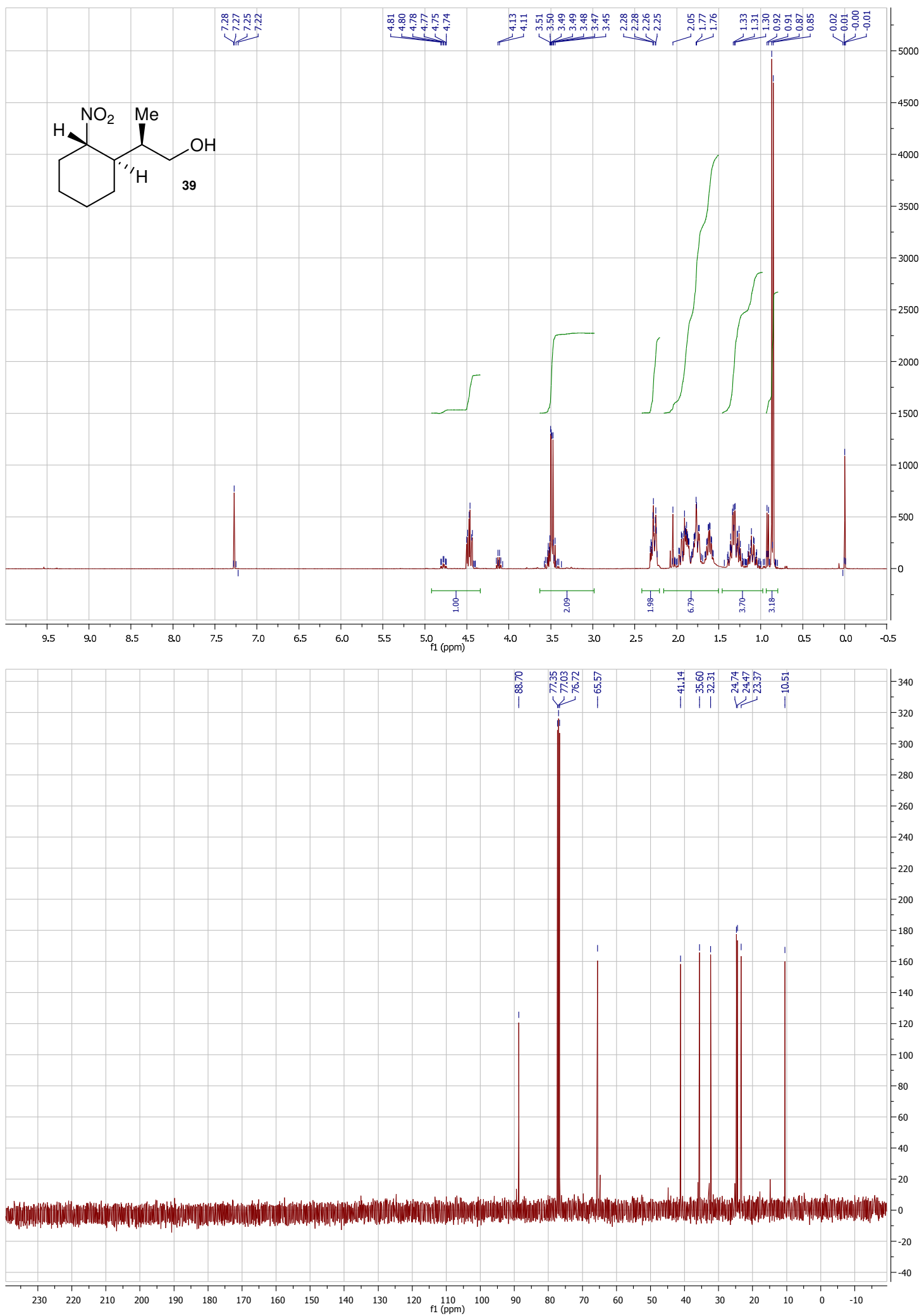




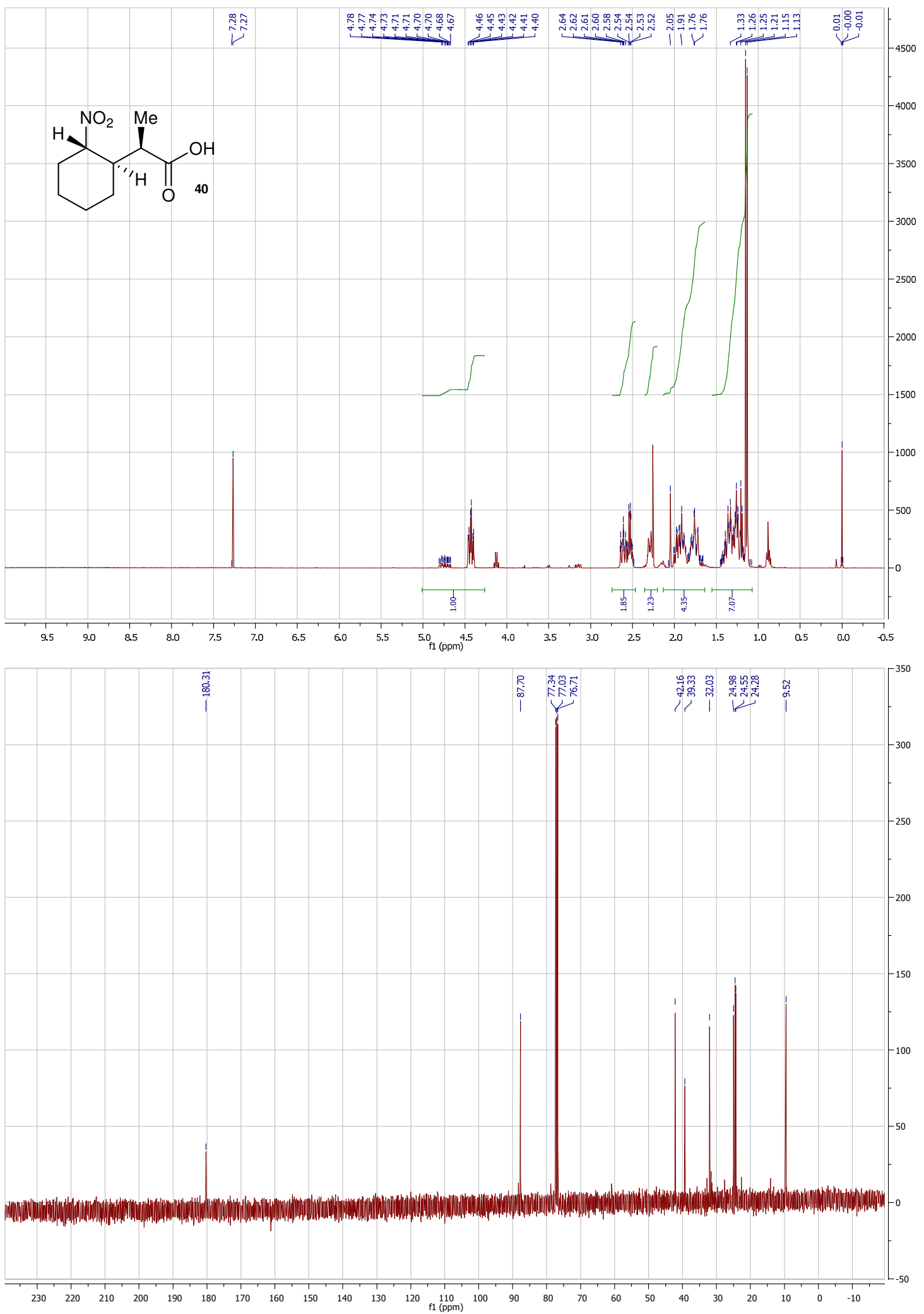



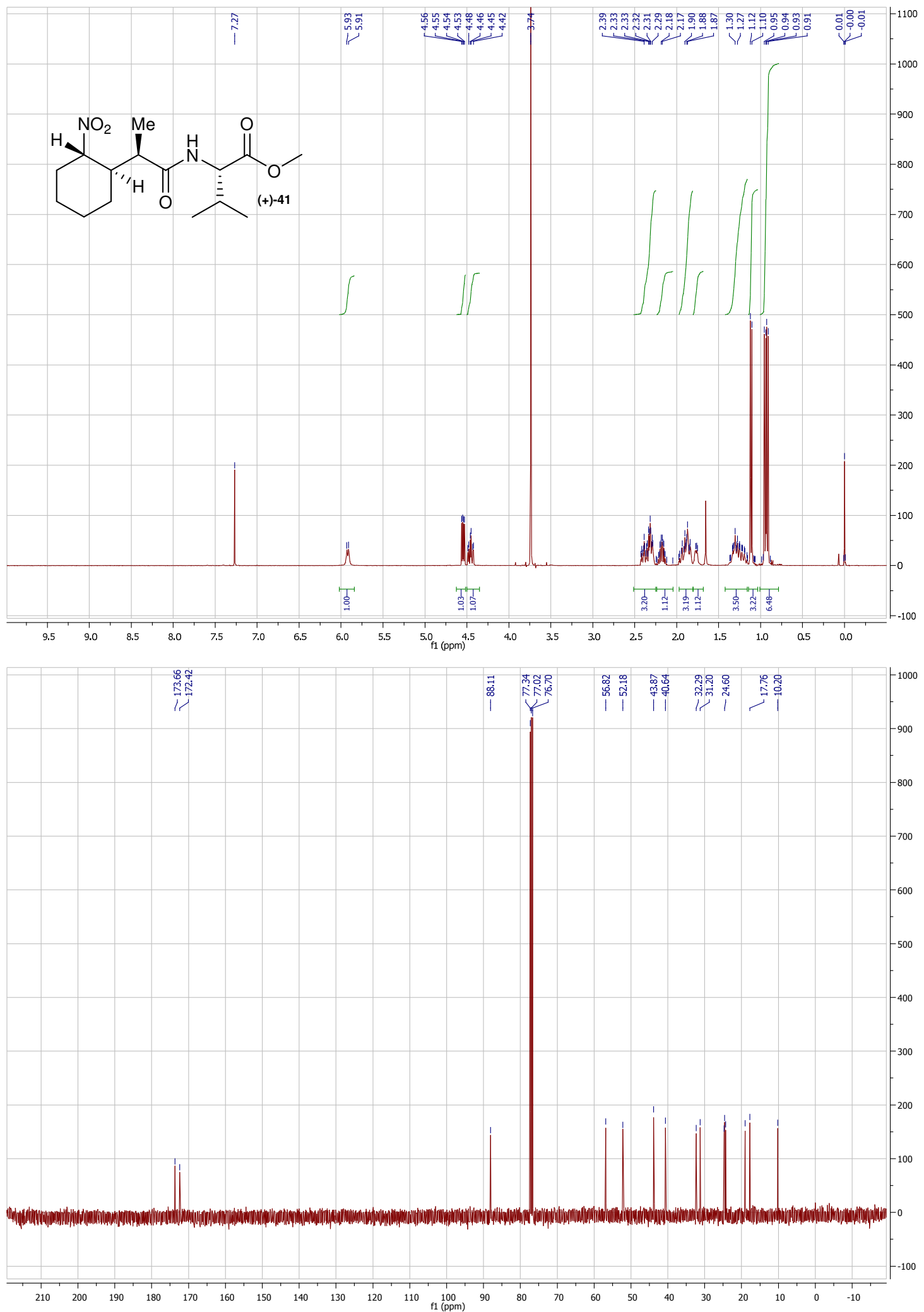


\section{X-Ray Crystal Structure and Associated Data for Compound X}

All $\mathrm{H}$ atoms were located in difference Fourier maps but were placed geometrically after each cycle of the refinement at a distance of $0.95 \AA$ from the connected atom. Isothermal temperature factors at $1.2 \mathrm{x} u$ iso of the attached atom.

Data collection: CrysAlis CCD (Oxford Diffraction, 2006); cell refinement: CrysAlis RED (Oxford Diffraction, 2006); data reduction: CrysAlis RED; program(s) used to solve structure: SIR92 (Altomane et al., 1994); program(s) used to refine structure; CRYSTALS (Betteridge et al., 2003); molecular graphics: CAMERON (Watkin et al., 1996); software used to prepare material for publication: CRYSTALS.

\begin{tabular}{|l|l|}
\hline Formula & $\mathrm{C}_{9} \mathrm{H}_{15} \mathrm{~N}_{1} \mathrm{O}_{1}$ \\
\hline$M_{\mathrm{r}}$ & 153.22 \\
\hline Crystal habit & colourless block \\
\hline Crystal dimensions $/ \mathrm{mm}$ & $0.10 \times 0.15 \times 0.30$ \\
\hline Crystal system & monoclinic \\
\hline Space group & $P 2_{1}$ \\
\hline$T / \mathrm{K}$ & $295(1)$ \\
\hline$a / \AA$ & $5.3337(3)$ \\
\hline$b / \AA$ & $8.4781(5)$ \\
\hline$c / \AA$ & $10.0357(6)$ \\
\hline$\alpha /^{\circ}$ & 90 \\
\hline$\beta /{ }^{\circ}$ & $99.050(6)$ \\
\hline$\gamma /{ }^{\circ}$ & 90 \\
\hline $\mathbf{V} / \AA^{3}$ & $448.16(5)$ \\
\hline$Z$ & 2 \\
\hline$\lambda / \AA$ & 0.71073 \\
\hline$\mu / \mathrm{mm}^{-1}$ & 0.578 \\
\hline$\rho_{\text {calc }} / \mathrm{Mg} \mathrm{m}^{-3}$ & 1.135 \\
\hline$R(F),{ }_{w} R(F)$ & $0.0321,0.0376$ \\
\hline & \\
\hline & \\
\hline
\end{tabular}


asymmetric unit with ellipsoids drawn at $50 \%$ probability

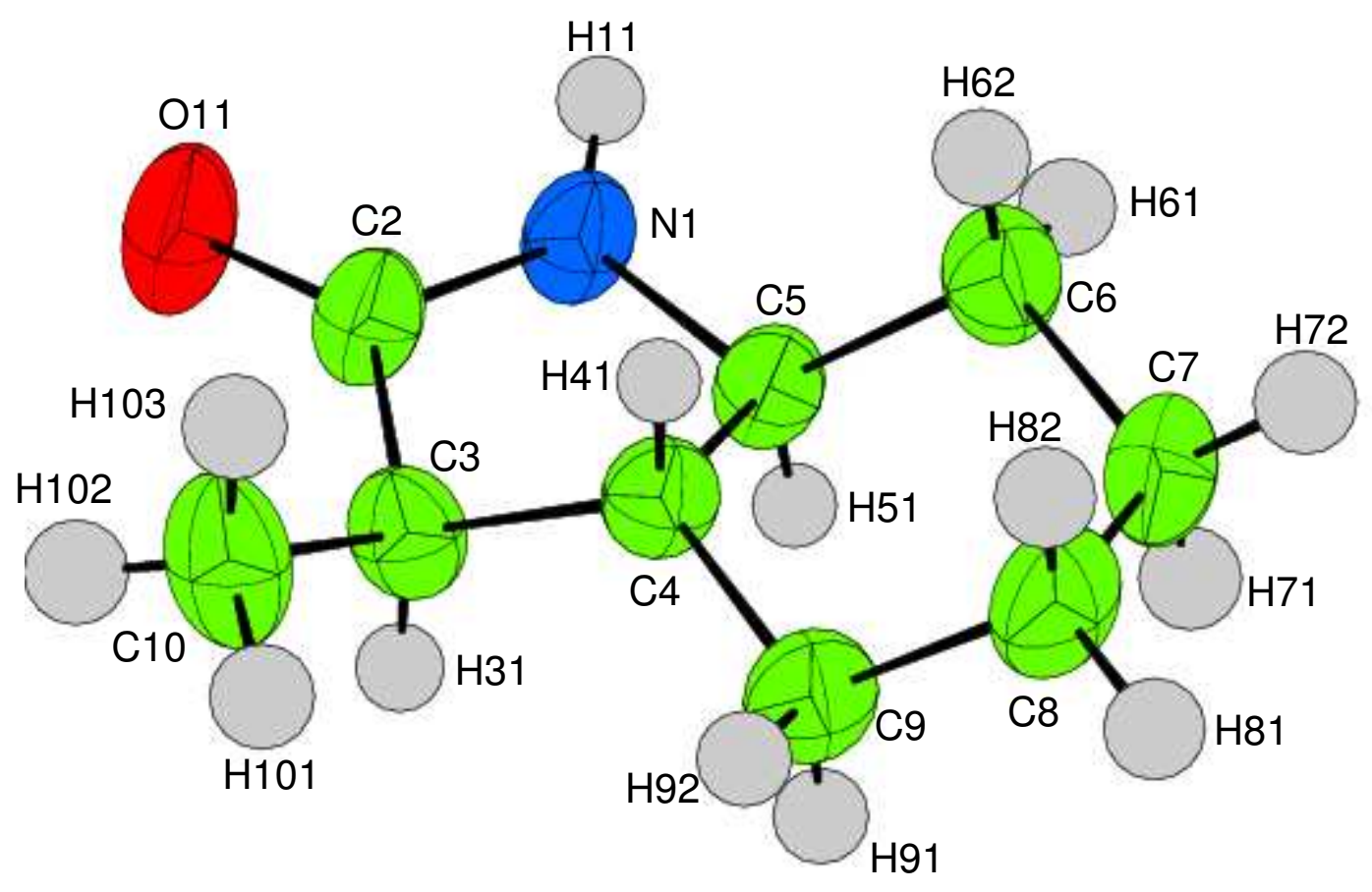


view along $a$ axis showing hydrogen bonding interactions (as dotted lines) between N1 and O11 of neighbouring molecules

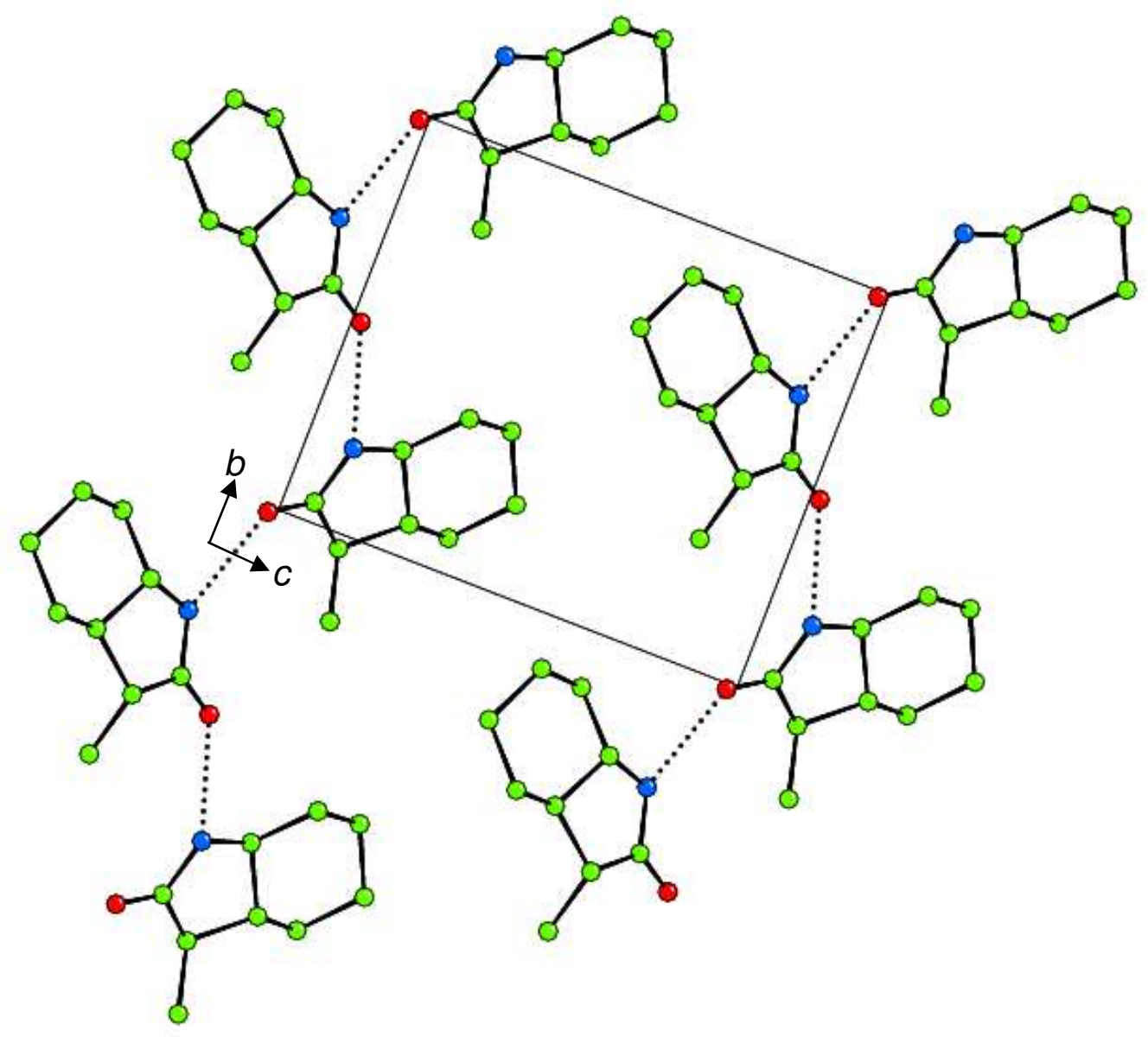

